\title{
O Problema de Stefan unidimensional
}

\author{
Arthur Miranda do Espírito Santo \\ DISSERTAÇÃO APRESENTADA \\ $\mathrm{AO}$ \\ Instituto DE MATEMÁticA E EstatísticA \\ DA \\ UNIVERSIDADE DE SÃo PAUlO \\ PARA \\ OBTENÇÃO DO TÍTULO \\ DE \\ Mestre em CiÊnCIAS \\ Programa: Matemática Aplicada \\ Orientador: Prof. Dr. Sérgio Muniz Oliva Filho
}

Durante o desenvolvimento deste trabalho o autor recebeu auxílio financeiro do CNPq

São Paulo, maio de 2013 


\section{O Problema de Stefan unidimensional}

Esta versão da dissertação contém as correções e alterações sugeridas pela Comissão Julgadora durante a defesa da versão original do trabalho, realizada em 06/05/2013. Uma cópia da versão original está disponível no

Instituto de Matemática e Estatística da Universidade de São Paulo.

Comissão Julgadora:

- Prof. Dr. Sergio Muniz Oliva Filho (orientador) - IME-USP

- Prof. Dr. Nelson Mugayar Kuhl - IME-USP

- Prof $^{\mathrm{a}}$. Dr ${ }^{\mathrm{a}}$. Claudia Garcia - EACH-USP 
Do not worry about your difficulties in Mathematics. I can assure you that mine are still greater. Albert Einstein 


\section{Agradecimentos}

Sou extremamente grato ao meu professor orientador Sérgio Muniz Oliva Filho que há muito tempo, desde a minha remota época de graduação, vem me ajudando a crescer no meio acadêmico e matemático, através de sua paciência e serenidade. Seu papel foi fundamental na organização de minhas ideias.

Sou grato à minha família, em especial ao meu pai Adelson, minha mãe Hilde e minha irmã Alice, que sempre aguentam meus momentos de mau humor, mas que sempre comemoram comigo meus êxitos. Ao meu primo-irmão Filipe que cresceu comigo. À minha família de longe, lá de Belém: tias, tios, primas, primos, avós, que sei que mesmo longe vivem torcendo por mim.

Sou grato aos meus amigos Bárbara e Bernard, a quem tenho como irmãos e que estão presentes, mesmo longe, nas fases mais importantes da minha vida. Sou grato também ao meu amigo Lyan que mesmo no caos e confusão de ideias consegue me entender muito bem.

Sou grato aos meus amigos Ariane, Rafael e Felipe meus companheiros de ciências do colégio e agora amigos da vida toda. E sou grato aos outros amigos do colégio que não são tão fãs assim de Matemática, como Eizo, Akira, Gabi, Fer, João, Maiara, Diego, Patty, Michele, Karina, André, Milene, Gary... Para cada um deles, em algum momento de suas vidas já estudamos matemática juntos.

Sou grato aos meus amigos psicólogos Rodrigo e Rafaela, com quem trabalhei, mas que hoje são dois amigos indispensáveis, a quem devo muito por sua sabedoria emocional.

Sou grato ao IME e à sua comunidade: amigos, professores, funcionários, que há mais de seis anos se tornaram parte inerente à minha vida. Aos professores Nelson, Orlando, Helena, Cordaro, Soto e Toscano, professores que tenho como modelos de pessoas e educadores. Aos colegas Bruno, Priscila, Thiago, Maria, Roberto, Thiago, Adele e Max.

Sou grato aos amigos da USP em geral, da Letras, da ECA, que sempre me deram insights muito além do pensamento das exatas. Aos colegas Miguel, Samuel, Ricardo, Julian y Mayra, Bruna, Francine, Ana...

Sou grato a muitas pessoas aqui não citadas, mas que foram igualmente importantes pra mim. Sem o apoio de tanta gente este trabalho não seria possível. 


\section{Resumo}

SANTO, A. M. E. O Problema de Stefan unidimensional. 2013. 86 f. Dissertação (Mestrado) - Instituto de Matemática e Estatística, Universidade de São Paulo, São Paulo, 2013.

O seguinte trabalho procura estudar problemas de fronteira móvel, conhecidos por problemas de Stefan, bem como aproximar suas soluções. Aplicações de problemas de Stefan encontram-se, por exemplo, na física termal de mudança de estados, presente em diversos fenômenos físicos e químicos naturais e na indústria. Devido a não-linearidade, a maior parte destes problemas não possuem solução analítica conhecida e uma técnica comum para se aproximar soluções é o método de balanceamento integral, inicialmente estudado por Goodman (1958). Este método e suas variações propõem perfis de aproximação no domínio da solução e resolvem uma versão integral da equação diferencial. O problema se resume a resolver uma equação diferencial ordinária no tempo envolvendo a profundidade de penetração do calor e o perfil de aproximação proposto. O trabalho estuda tais métodos para problemas termais clássicos em primeiro lugar, de modo que a extensão para problemas de Stefan seja natural. Refinamentos são apresentados, bem como uma técnica de subdivisão do espaço que resulta num esquema numérico. A técnica de imobilização e fronteira é desenvolvida e aplicada em diversos momentos, a fim de simplificar a utilização dos métodos integrais.

Palavras-chave: Problema de Stefan, Métodos de Balanceamento Integral, Fronteira móvel. 


\section{Abstract}

SANTO, A. M. E. The one-dimensional Stefan Problem. 2013. 86 f. Dissertação (Mestrado) Instituto de Matemática e Estatística, Universidade de São Paulo, São Paulo, 2013.

The current work aims to study moving boundary problems, known as Stefan problems, and approximate their solutions. Applications of Stefan problems are found in situations where there is change of physical state, present in several natural and industrial physical and chemical phenomena. Due to their inherent nonlinearity, most of these problems have no known analytic solution and a common technique to approximate solutions is the heat balance integral method, originally studied by Goodman (1958). This method and its variations propose an approximating profile and solve an integral version of the differential equation. The problem is reduced to solving an ordinary differential equation in time involving the depth of heat penetration and the proposed profile. This work studies such classic methods to thermal problems first, in a way that the extension to Stefan problems is natural. Refinements are presented, as well as a technique of subdividing the space domain which results in a numerical scheme. The technique of boundary immobilization is developed and applied at different times in order to simplify the use of these methods.

Keywords: Stefan Problem, Heat Balance Integral Methods, Moving Boundary. 


\section{Sumário}

$\begin{array}{lc}\text { Lista de Abreviaturas } & \text { ix }\end{array}$

Lista de Símbolos $\quad$ xi

Lista de Figuras $\quad$ xiii

Lista de Tabelas $\quad$ xv

1 Introdução 1

2 O Problema Termal 5

2.1 Solução Analítica . . . . . . . . . . . . . . . . . . . . . . . 5 5

2.2 Soluções Aproximadas . . . . . . . . . . . . . . . . . . . . . . . . 6

$2.2 .1 \quad$ O Método de Balanceamento Integral _ . . . . . . . . . . . . . . . 6

2.2.2 O Método de Balanceamento Integral Refinado . . . . . . . . . . . . . . . . 12

2.2 .3 A escolha do expoente $m \ldots \ldots \ldots \ldots \ldots$

2.2.4 Refinamento usando subdivisões espaciais - o Método Nodal . . . . . . . . . . 15

3 O Problema Termal Revisitado $\quad 19$

3.1 Condições de contorno . . . . . . . . . . . . . . . . . . . . . . . . . . . . 19

3.1 .1 Fluxo constante na fronteira . . . . . . . . . . . . . . . . . . . . . 19

3.1 .2 Condição de Resfriamento . . . . . . . . . . . . . . . . . . . . . . . . . 23

3.2 Imobilização de fronteira . . . . . . . . . . . . . . . . . . . . . . . 26

3.2 .1 Fluxo constante na fronteira . . . . . . . . . . . . . . . . . . 29

3.2 .2 Condição de Resfriamento . . . . . . . . . . . . . . . . . . . . . . . 30

3.3 Imobilização de Fronteira e Subdivisões Espaciais . . . . . . . . . . . . . . . . . . 32

3.3.1 Condição de temperatura constante na fronteira . . . . . . . . . . . . . . 33

3.3.2 Fluxo constante . . . . . . . . . . . . . . . . . . . . . . . 37

3.3 .3 Condição de Resfriamento . . . . . . . . . . . . . . . . . . . . . . . . . 39

3.3.4 Condição de Dirichlet . . . . . . . . . . . . . . . . . . . . . . . . . 39

4 O Problema de Stefan $\quad 41$

4.1 Apresentação do problema e o Problema unidimensional de uma fase. . . . . . . . . . 41

4.2 Soluções Aproximadas . . . . . . . . . . . . . . . . . . . . . . . . 44

$4.2 .1 \quad$ O método de Balanceamento Integral . . . . . . . . . . . . . . . . . 44

$4.2 .2 \quad$ O método de Balanceamento Integral Refinado . . . . . . . . . . . . . . . . 52 
viii SUMÁRIO

4.2 .3 O problema de Stefan imobilizado . . . . . . . . . . . . . . . 54

4.2.4 Refinamento usando subdivisões espaciais - o método Nodal . . . . . . . . . . 56

4.3 Outras Aproximações Numéricas . . . . . . . . . . . . . . . . . . . . . . . . . . 59

4.3.1 O método Variable Space Grid (VSG) . . . . . . . . . . . . . . . . 59

4.3.2 O método de fronteira imobilizada $(\mathrm{BIM}) \ldots \ldots \ldots$

5 Resultados e Considerações Finais $\quad 63$

$\begin{array}{ll}\text { Referências Bibliográficas } & 69\end{array}$

$\begin{array}{ll}\text { Índice Remissivo } & 71\end{array}$ 


\section{Lista de Abreviaturas}

HBIM Método de Balanceamento Integral do Calor

(Heat Balance Integral Method)

RIM Método de Balanceamento Integral do Calor Refinado

(Refined Heat Balance Integral Method)

CIM Método de Balanceamento Integral do Calor Combinado

(Combined Heat Balance Integral Method) 


\title{
Lista de Símbolos
}

\author{
$\rho \quad$ Densidade do material em $\frac{\mathrm{kg}}{\mathrm{m}^{3}}$ \\ $K \quad$ Condutividade Térmica em $\frac{W}{m K}$ \\ $c_{p} \quad$ Calor Específico em $\frac{k J}{k g K}$ \\ $L_{m} \quad$ Calor Latente de Derretimento em $\frac{J}{k g}$
}




\section{Lista de Figuras}

2.1 Solução do Problema Termal . . . . . . . . . . . . . . . . . . 6

2.2 Curvas de nível da solução do Problema Termal . . . . . . . . . . . . . . . . . . 6

2.3 Aprox. da solução do problema termal via HBIM . . . . . . . . . . . . . . . 8

2.4 Curvas de nível da aprox. da solução do problema termal via HBIM . . . . . . . . . . 9

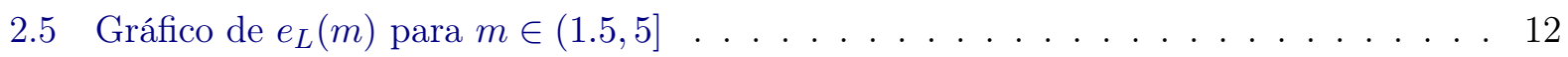

2.6 Gráfico de $e_{L}(m) \ldots \ldots \ldots \ldots \ldots \ldots \ldots \ldots \ldots$

2.7 Gráfico da solução $u(x, t)$ em $t=1$ e aproximações $\ldots \ldots \ldots \ldots$. . . . . . . . 14

3.1 Gráfico da solução exata do problema $3.1 \ldots \ldots$. . . . . . . . . . . . 20

3.2 Curvas de nível da solução exata do problema $3.1 \ldots \ldots$. . . . . . . . . . . 20

3.3 Aproximação via HBIM com fluxo constante na fronteira e $m=4$ obtido via CIM . . 21

3.4 Curvas de nível da aproximação via HBIM com fluxo constante na fronteira e $m=422$

3.5 Aproximação via RIM com fluxo constante na fronteira e $m=3.822$ obtido via minimização do erro de Langford . . . . . . . . . . . . . . . . . . . 22

3.6 Curvas de nível da aproximação via RIM com fluxo constante na fronteira e $m=3.82222$

3.7 Gráfico da solução do problema $3.3 \ldots \ldots$. . . . . . . . . . . . . 23

3.8 Curvas de nível da solução do problema $3.3 \ldots \ldots$. . . . . . . . . . . . . 23

3.9 Gráfico de $\delta(t)$ para o problema termal com condição de resfriamento (obtido numericamente a partir do método CIM) . . . . . . . . . . . . . . 25

3.10 Curva do expoente $m$ dependente do tempo obtido numericamente a partir do método CIM para o problema termal com condição de resfriamento . . . . . . . . . . . 25

3.11 Gráfico da aproximação via CIM do problema 2.1 com condição de resfriamento . 26

3.12 Curvas de nível da aproximação via CIM problema 2.1 com condição de resfriamento 26

$3.13 E_{M}$ de HBIM e RIM . . . . . . . . . . . . . . . . . . . . . . . 29

3.14 Gráfico das funções $f_{i}(\xi), \operatorname{com} \xi \in[0,1]$. Estão destacados os cinco pontos nodais $U_{i} \quad 37$

3.15 Gráfico da aproximação via HBIM nodal $(n=4)$ com temperatura constante na

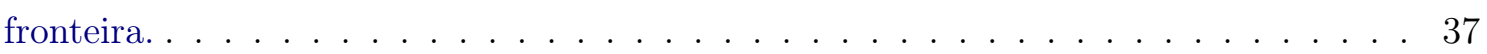

3.16 Curvas de nível da aproximação via HBIM nodal $(n=4)$ com temperatura constante na fronteira. . . . . . . . . . . . . . . . . . . . . . . 38

4.1 Solução do Problema de Stefan com temperatura constante na fronteira e $\beta=1$. . . 44

4.2 Curvas de nível da solução do Problema de Stefan com temperatura constante na

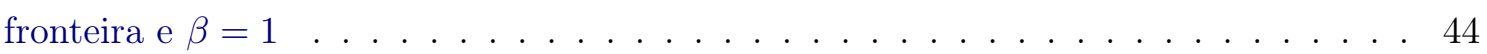

5.1 Gráfico da solução do problema 2.1 com temperatura constante na fronteira . . . . . 65 


\section{Lista de Tabelas}

3.1 Imobilização de Fronteira para diferentes condições . . . . . . . . . . . . . . . . . 32

$5.1 \alpha$ aproximado para distintos valores de $\beta$ em problemas de Stefan com condição de temperatura constante na fronteira . . . . . . . . . . . . 63

5.2 erro percentual de $\alpha$ aproximado com relação a $\alpha$ real para distintos valores de $\beta$ em problemas de Stefan com condição de temperatura constante na fronteira . . . . . . . 63

5.3 erro percentual de $\alpha$ aproximado em relação a $\alpha$ real para distintos valores de $\beta$ em problemas com condição de Robin . . . . . . . . . . . . . . . . . . . . . 64

5.4 Erro máximo em $u(x, 1)$ para aproximações de soluções do problema com temperatura constante na fronteira . . . . . . . . . . . . . . . . . . . 65

5.5 Resultados para o problema de Stefan com temperatura constante na fronteira. . . . 65 


\section{Capítulo 1}

\section{Introdução}

Problemas de fronteira móvel (conhecidos por Problemas de Stefan) envolvendo condução de calor em conjunto com mudança de fase são de grande interesse em diversas áreas da ciência, engenharia e indústria. Tal processo cobre uma ampla extensão de aplicações em que há mudança de estado físico. Os problemas de fronteira móvel ocorrem em muitas áreas como nas indústrias de metal, vidro, plástico e petróleo, no desenho de espaçonaves, na preservação de bens perecíveis, em processos químicos e difusivos, etc. Assume-se que o material passa por uma mudança de fase com uma fronteira móvel em que a posição exata é desconhecida e deve ser encontrada como parte da análise. Como problemas de fronteira móvel requerem resolver a equação do calor em uma região que também deve ser determinada como parte da solução, eles são inerentemente não-lineares. Devido a essa não-linearidade, tais problemas podem ser resolvidos analiticamente apenas para um número limitado de casos especiais. Assim, diversas técnicas de aproximação semi-analíticas de soluções foram desenvolvidas, bem como métodos numéricos de diferenças finitas.

A técnica central estudada neste trabalho é o método de balanceamento integral (HBIM). Esse método é uma técnica simples de aproximação originalmente desenvolvida para analisar problemas termais. Goodman (1958) foi o primeiro a introduzir o método. Porém, como os problemas termais de domínios fixos possuem soluções analíticas, o método encontrou seu maior uso em problemas de Stefan.

O método HBIM padrão aproxima as soluções dos problemas ao introduzir uma profundidade de penetração do calor, $\delta(t)$, em que para $x \geq \delta(t)$ a mudança de temperatura com relação ao estado inicial é supostamente negligenciável. Um perfil de aproximação é então definido para a temperatura, tipicamente um polinômio, e, aplicando uma quantidade suficiente de condições de fronteira, os coeficientes podem ser determinados em função da variável $\delta$. Finalmente, a equação do problema é integrada sobre o domínio $[0, \delta]$ para produzir a equação do balanceamento integral, uma equação diferencial para $\delta$. O problema reduz-se a resolução de uma equação diferencial ordinária de primeira ordem no tempo. Embora esse método não seja tão preciso em alguns casos como em outros métodos numéricos, ele ainda é uma escolha popular devido a sua simplicidade. Ao se resolver um problema de Stefan, a abordagem é similar, com a exceção de que a fronteira móvel é agora uma variável do problema. A condição de Stefan proporciona mais uma equação a fim de determinar essa variável.

Um refinamento proposto para esse método é o Método de Balanceamento Integral Refinado (RIM). É uma abordagem alternativa, onde a equação do calor é integrada duas vezes, em vez de uma. Em certos casos, o RIM pode melhorar a solução do HBIM, mas não é possível escolher 
o melhor método para todos os casos. Os méritos de cada método são discutidos no trabalho de Mitchell e Myers (2010a).

O método HBIM tem algumas desvantagens conhecidas. Por exemplo, ele pode ser apenas aplicado a problemas unidimensionais e a escolha do perfil de aproximação é arbitrária. Essa escolha é uma constante fonte de debate, e Goodman (1958) usou inicialmente um perfil quadrático. O trabalho de Mosally et al. (2002) sugere um perfil exponencial, dada a natureza da solução de problemas com solução analítica. Caso seja escolhido um perfil polinomial, ainda há a discussão sobre que grau esse polinômio deve ter. Para isso, Langford (1973) introduz a noção de erro baseado em mínimo quadrados, de forma que o expoente que minimiza esse erro seria ideal. Mitchell e Myers (2012), contudo, apresenta algumas desvantagens dessa formulação e propõe um novo olhar para o erro, fazendo uso da técnica de imobilização de fronteira.

Outra melhoria proposta para o método é um refinamento que utiliza subdivisões espaciais. Nele, a equação integral é estudada para várias subdivisões do domínio, a fim de aumentar a precisão da aproximação. O uso de perfis lineares nessas subdivisões foi inicialmente estudado por Bell e Abbas (1985), mas a linearidade dos perfis implica num aumento de sub-regiões, fazendo o método HBIM nodal se tornar um procedimento numérico. Mas o trabalho de Mitchell (2011) consegue obter alta precisão mesmo com um pequeno número de sub-regiões, tanto para problemas termais quanto para problemas de Stefan. Em seu trabalho, Mitchell utiliza perfis cúbicos suaves e o caso quadrático é brevemente estudado.

Dadas essas considerações preliminares, o presente trabalho visa apresentar algumas das diversas técnicas empregadas na solução de problemas termais e suas extensões para problemas de Stefan. Os métodos apresentados são o método de balanceamento integral (HBIM), o método de balanceamento integral refinado (RIM) e o método de balanceamento integral nodal, que se utiliza de subdivisões espaciais. A utilização desses métodos em problemas termais é principalmente para extensão para problemas de Stefan, visto que problemas termais possuem soluções analíticas. São discutidas duas maneiras alternativas de avaliar a precisão de uma solução aproximada. Todos os métodos são também revistos sob a ótica da técnica de imobilização de fronteira, em que o tratamento do problema fica simplificado. São analisadas também várias condições de fronteira, além da temperatura constante, como a condição de fluxo constante, a condição de resfriamento, a condição de Robin genérica e a condição de Dirichlet genérica. Além disso, diferentes perfis de aproximação também são analisados, e no final são apresentadas algumas conclusões sobre vantagens e desvantagens das escolhas empregadas. 


\section{Organização do Trabalho}

No capítulo 2 é estudado o problema termal. Nele são desenvolvidos os métodos HBIM e RIM. Um perfil de aproximação polinomial genérico é proposto e são apresentadas comparações com a solução exata, uma vez que essas existem para problemas termais. Além disso, são introduzidas maneiras de medir a precisão dos métodos, como o erro definido por Langford (1973) e as melhorias propostas por Mitchell e Myers (2012). Esse último trabalho é a referência central para os tópicos citados, e é uma recente publicação sobre o assunto.

Segue-se uma breve discussão sobre a escolha do expoente do perfil de aproximação polinomial e a introdução do método Combined Integral Method (CIM), ou método integral combinado, em que combinam-se os métodos HBIM e RIM para determinar tal expoente.

O capítulo termina com a introdução de um método que propõe subdivisões no domínio espacial do problema, em que perfis de aproximação polinomiais são usados em cada sub-região A referência para este método é Mitchell (2011).

O capítulo 3 ainda versa sobre o problema termal. Mas neste capítulo são propostas aproximações de problemas termais com condições de fronteira variadas, diferentemente do capítulo 2, que se preocupava apenas com o problema com condição de fronteira de temperatura constante. São encontradas aproximações via HBIM e RIM para problemas com condição de fronteira de fluxo constante ou com condição de resfriamento. As maneiras de medir a precisão dos métodos introduzidas no capítulo 2 são revisitadas para as novas condições de fronteira.

A seguir, é apresentada a técnica de imobilização de fronteira, fundamental no estudo dos métodos subsequentes. O problema termal é estudado sob uma nova ótica, em que a fronteira móvel agora é estática, como é evidente no nome da técnica. A referência para essas duas últimas partes ainda é Mitchell e Myers (2012).

Ao final do capítulo aplica-se essa técnica para o método refinado com subdivisões espaciais. É apresentada, pela primeira vez, uma condição de fronteira de Dirichlet genérica para o problema termal. Novamente, para esta seção segue-se o estudo de Mitchell (2011).

O capítulo 4 começa com uma introdução histórica sobre o problema de Stefan, seguindo o trabalho de Crepeau (2007). Nesta introdução é desenvolvida a modelagem do problema, e apresentada sua forma atual. O problema estudado possui uma mudança de fase, e, portanto, uma fronteira móvel. Essa característica é fundamental para as analogias dos métodos desenvolvidos para problemas termais com os problemas de Stefan.

As ideias do trabalho de Mitchell e Myers (2010a) são expostas nesse capítulo através do estudo do método HBIM e do método RIM para condições de fronteira com temperatura constante ou para condições de fronteira de Robin (que generaliza a condição de fluxo constante e de resfriamento). Os perfis de aproximação estudados são quadráticos, cúbicos e exponenciais, esses últimos inéditos até o momento no trabalho.

Na parte seguinte estuda-se o problema de Stefan imobilizado, com um perfil polinomial de grau $m$ livre, e a técnica de imobilização de fronteira do capítulo anterior é facilmente aplicada para esse caso.

O método refinado com subdivisões espaciais é revisto para problemas de Stefan através de duas exposições. A primeira é uma técnica similar ao estudo inicial da técnica de aproximações em subdivisões espaciais do problema termal, desenvolvida por Bell e Abbas (1985) e estudada por Mosally et al. (2005). A segunda é a continuação do estudo para problemas termais com imobili- 
zação de fronteira e subdivisões espaciais, feita por Mitchell (2011).

O capítulo termina com duas técnicas de diferenças finitas para solução de problemas de Stefan, o método VSG (Variable Space Grid) e o método BIM (Boundary Immobilization Method). Esses métodos foram incluídos no trabalho devido a sua similaridade com os métodos de subdivisões espaciais; o método BIM nada mais é que o próprio método VSG com a fronteira imobilizada. Para essa seção, as referências principais são os trabalhos de Kutluay et al. (1997) e de Savović e Caldwell (2009).

Seguem-se as considerações finais sobre o trabalho, em que são apresentados alguns resultados de experimentos de aproximação e numéricos, algumas extensões naturais para os métodos apresentados e comentários sobre problemas de Stefan com mais de uma mudança de fase. 


\section{Capítulo 2}

\section{O Problema Termal}

Um problema físico bastante estudado é o aquecimento de um sólido semi-infinito, com uma temperatura constante especificada em uma de suas fronteiras. Apesar de esse problema possuir soluções exatas para diferentes condições de fronteira e de contorno, o estudo das seguintes técnicas será crucial para a aplicação das mesmas sobre problemas de Stefan, que normalmente não possuem soluções exatas.

Supondo a fronteira o ponto $x=0$, na forma adimensionalizada, uma versão bem simples do problema pode ser escrita como

$$
\begin{aligned}
\frac{\partial u}{\partial t}-\frac{\partial^{2} u}{\partial x^{2}} & =0, \quad 0<x<\infty \\
u(x, 0) & =0 \\
u(0, t) & =1 \\
u & \rightarrow 0, \quad x \rightarrow \infty
\end{aligned}
$$

\subsection{Solução Analítica}

O problema possui a solução exata

$$
u(x, t)=1-\frac{2}{\sqrt{\pi}} \int_{0}^{\frac{x}{2 \sqrt{t}}} e^{-\tau^{2}} d \tau:=\operatorname{erfc}\left(\frac{x}{2 \sqrt{t}}\right)
$$

Mesmo com $\lim _{t \rightarrow \infty} \operatorname{erfc} \frac{x}{2 \sqrt{t}}=1$, tem-se $\lim _{x \rightarrow \infty} \operatorname{erfc} \frac{x}{2 \sqrt{t}}=0$ para $t$ fixado. A última condição do problema proposto é, portanto, satisfeita. A seguir um gráfico da solução e das suas curvas de nível. 


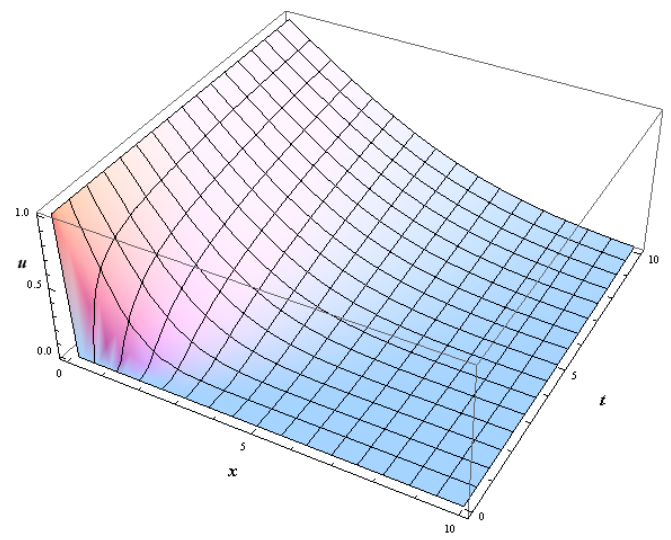

Figura 2.1: Gráfico da solução do problema 2.1 com $(x, t) \in(0,10) \times(0,10)$

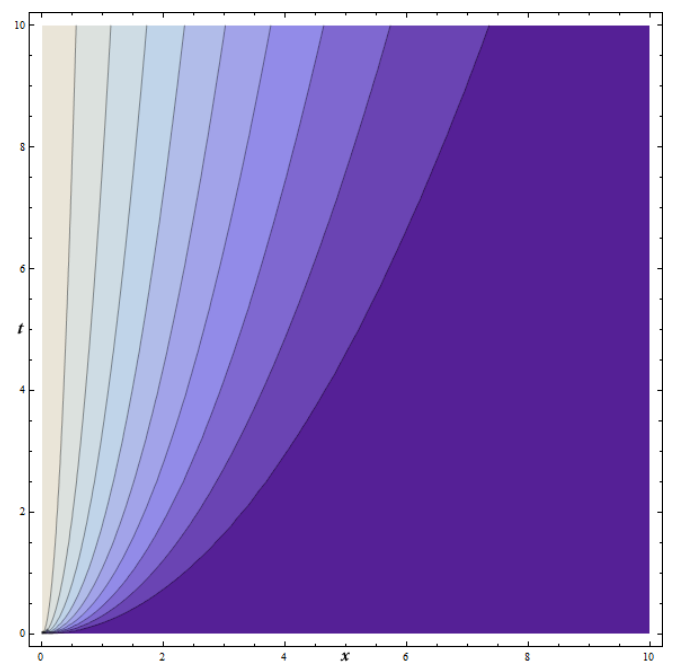

Figura 2.2: Curvas de nível da solução do problema 2.1 com $(x, t) \in(0,10) \times(0,10)$

\subsection{Soluções Aproximadas}

Nesta seção são apresentadas algumas técnicas de aproximação da solução do problema 2.1. A teoria de soluções fracas para uma equação parabólica pode ser vista com mais profundidade no livro de Evans (1998). As soluções aproximadas apresentadas nesse trabalho satisfazem não necessariamente a equação do calor, e sim uma versão integral da mesma.

\subsubsection{O Método de Balanceamento Integral}

A solução do problema 2.1 satisfaz a equação do calor e também satisfaz a seguinte equação:

$$
\int_{0}^{\infty} \frac{\partial u}{\partial t}-\frac{\partial^{2} u}{\partial x^{2}}=0
$$

Muitas aproximações não satisfazem a equação do calor mas satisfazem essa equação integral, que é simplesmente um "balanço" entre a energia de entrada do lado esquerdo contra seu efeito mensurável na direita. Assume-se que, em um determinado tempo $t$, o efeito de uma perturbação na fronteira esquerda (dada pela condição de contorno) não é significativo além de uma distância finita $x=\delta(t)$ e a partir desse ponto vale a condição de fronteira à direita. Para o problema atual, 
essa suposição pode ser matematicamente escrita como

$$
u(\delta(t), t)=0
$$

O procedimento de aproximação se resume a encontrar uma função $u(x, t)$ que satisfaz as condições de fronteira (que para esse caso são $u(0, t)=1$ e $u(\delta(t), t)=0$ ) e $\delta(t)$ é encontrado a partir da imposição de que essa aproximação satisfaz a equação integral sujeita à condição inicial. Essas aproximações são tipicamente polinômios.

Resumindo, esse método clássico, inicialmente proposto por Goodman (1958), envolve três passos:

1. Define-se a profundidade de penetração da temperatura $\delta(t)$. Para $x \geq \delta(t)$, a mudança da temperatura com respeito a seu estado inicial é desprezível

2. É introduzido um perfil de aproximação, tipicamente polinomial, que descreve a temperatura para $0 \leq x \leq \delta(t)$

3. A equação é integrada sobre $x \in[0, \delta]$ para produzir o balanceamento integral do calor (heat balance integral). A equação diferencial resultante é então resolvida.

Uma aproximação usual é a polinomial

$$
u=\left(1-\frac{x}{\delta}\right)^{m}
$$

Para calcular a solução do método HBIM, substitui-se a condição de contorno no infinito por

$$
u(\delta(t), t)=\frac{\partial u}{\partial x}(\delta(t), t)=0
$$

Duas condições de contorno são requeridas para substituir a única condição no infinito devido à introdução de uma nova incógnita, $\delta(t)$, onde $\delta(0)=0$. Esta aproximação satisfaz $u_{x}(\delta, t)=0$ desde que $m>1$. Além disso, uma outra condição sobre a curvatura é frequentemente aplicada:

$$
\frac{d u}{d t}(\delta(t), t)=\left.\left(\frac{\partial u}{\partial t}+\frac{\partial u}{\partial x} \frac{d \delta}{d t}\right)\right|_{x=\delta}=0 \Rightarrow \frac{\partial^{2} u}{\partial x^{2}}(\delta(t), t)=0
$$

Caso essa condição seja imposta, deve-se ter $\frac{\partial^{2} u}{\partial x^{2}}(\delta, t)=0$, ou seja, $m>2$. De maneira geral, a escolha de $m$ é arbitrária, mas as escolhas mais comuns são $m=2$ ou 3. Resta determinar $\delta$. Integrando o lado esquerdo da equação diferencial com respeito a $x$ no domínio $[0, \delta(t)]$ e retirando a derivada em relação ao tempo para fora da integral, obtém-se:

$$
\int_{0}^{\delta} \frac{\partial u}{\partial t} d x=\frac{d}{d t} \int_{0}^{\delta} u d x-u(\delta(t), x) \delta^{\prime}(t)=\frac{d}{d t} \int_{0}^{\delta} u d x
$$

Já o lado direito fica

$$
\int_{0}^{\delta} \frac{\partial u^{2}}{\partial x^{2}} d x=-\left.\frac{\partial u}{\partial x}\right|_{x=\delta}-\left.\frac{\partial u}{\partial x}\right|_{x=0}=-\left.\frac{\partial u}{\partial x}\right|_{x=0}
$$


E assim obtém-se a equação do método HBIM

$$
\frac{d}{d t} \int_{0}^{\delta} u d x=-\left.\frac{\partial u}{\partial x}\right|_{x=0}
$$

Substituindo o perfil aproximado (2.6) na equação obtida, tem-se:

$$
\begin{aligned}
\frac{d}{d t} \int_{0}^{\delta}\left(1-\frac{x}{\delta}\right)^{m} d x & =-\left.\frac{\partial}{\partial x}\left(1-\frac{x}{\delta}\right)^{m}\right|_{x=0} \\
\left.\frac{d}{d t}\left(-\delta \frac{\left(1-\frac{x}{\delta}\right)^{m+1}}{m+1}\right)\right|_{0} ^{\delta} & =\left.\frac{m}{\delta}\left(1-\frac{x}{\delta}\right)^{m-1}\right|_{x=0} \\
\frac{d}{d t}\left(\frac{\delta}{m+1}\right) & =\frac{m}{\delta}
\end{aligned}
$$

A família de soluções dessa equação diferencial sob um parâmetro $k$ é dada por:

$$
\delta(t)=\sqrt{2 m(m+1) t+k}
$$

Supondo-se $\delta(0)=0$, chega-se à solução

$$
\delta(t)=\sqrt{2 m(m+1)} \sqrt{t}
$$

E portanto a solução aproximada do problema 2.1 é dada por

$$
u(x, t)= \begin{cases}\left(1-\frac{x}{\delta(t)}\right)^{m} & \text { se } 0 \leq x \leq \delta(t) \\ 0 & \text { c.c. }\end{cases}
$$

A seguir um exemplo para $m=2$. As figuras 2.3 e 2.4 mostram o gráfico da solução aproximada e o gráfico das curvas de nível da solução aproximada. É possível observar a curva $\delta(t)=\sqrt{12 t}$ destacada em branco no gráfico.

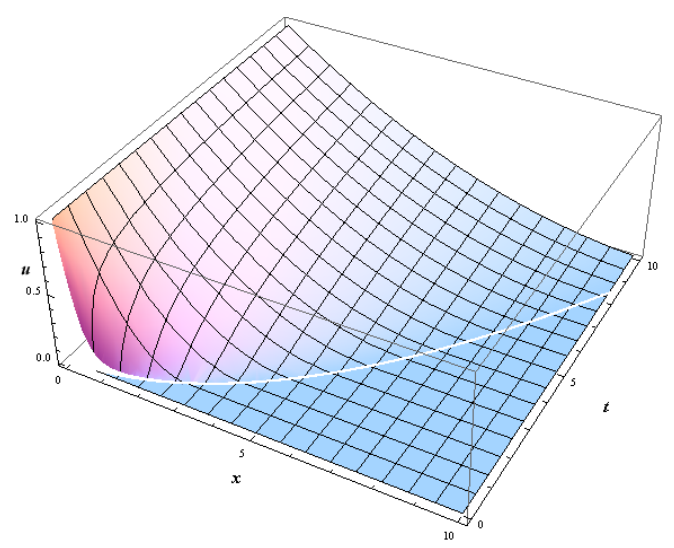

Figura 2.3: Gráfico da solução aproximada do problema termal com condição de temperatura constante na fronteira via HBIM com $(x, t) \in(0,10) \times(0,10)$. 


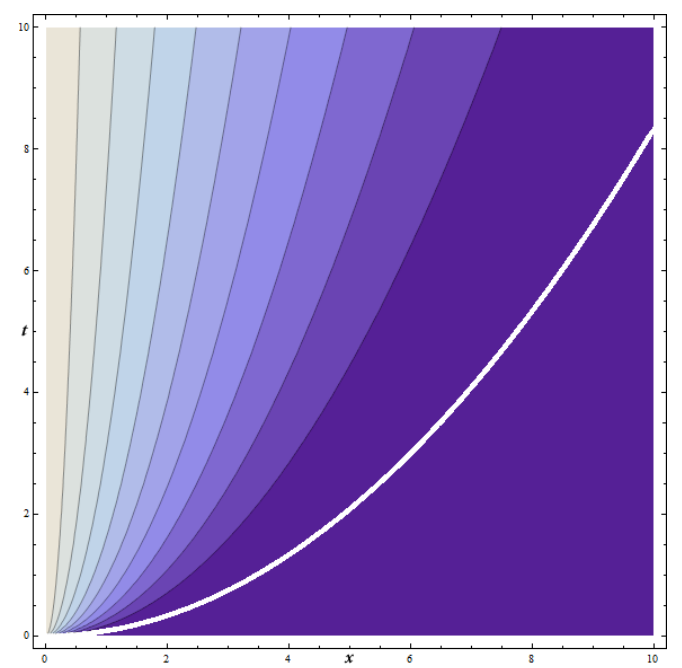

Figura 2.4: Curvas de nível da solução aproximada via $\operatorname{HBIM} \operatorname{com}(x, t) \in(0,10) \times(0,10)$. Valores mais baixos possuem cores mais escuras

\section{Comparação com a solução exata}

Considere a função $f(x, t)$ definida por

$$
f(x, t)=\frac{\partial u}{\partial t}-\frac{\partial^{2} u}{\partial x^{2}}
$$

Langford (1973) propõe um modelo de mínimos quadrados relativo à formulação do método HBIM em que

$$
E_{L}:=\int_{0}^{\delta} f(x, t)^{2} d x \geq 0
$$

$E_{L}$ é dita a estimativa de energia de $u$ para o problema termal. Se $u(x, t)$ é a solução exata da equação do calor, então tanto $f$ quanto $E_{L}$ são nulas. Entretanto, com o método HBIM, a equação do calor é satisfeita apenas num sentido integral e $f(x, t) \neq 0$ para todo $x$. Ou seja, a equação do calor não é satisfeita pela aproximação polinomial de $u$ e tem-se para o erro que $E_{L}>0$.

Proposição 2.2.1 Para o perfil polinomial (2.6) a seguinte igualdade é satisfeita

$$
f(x, t)=\frac{m}{\delta(t)^{2}}\left(1-\frac{x}{\delta(t)}\right)^{m-2}\left(x \delta^{\prime}(t)\left(1-\frac{x}{\delta(t)}\right)-(n-1)\right)
$$

Demonstração Pela própria definição de $f(x, t)$, ao se substituir o perfil aproximado,

$$
\begin{aligned}
f(x, t) & =\frac{\partial u}{\partial t}-\frac{\partial^{2} u}{\partial x^{2}} \\
& =\frac{\partial}{\partial t}\left(1-\frac{x}{\delta(t)}\right)^{m}-\frac{\partial^{2}}{\partial x^{2}}\left(1-\frac{x}{\delta(t)}\right)^{m} \\
& =\frac{m x \delta^{\prime}(t)}{\delta(t)^{2}}\left(1-\frac{x}{\delta(t)}\right)^{m-1}-\frac{m(m-1)}{\delta(t)^{2}}\left(1-\frac{x}{\delta(t)}\right)^{m-2} \\
& =\frac{m}{\delta(t)^{2}}\left(1-\frac{x}{\delta(t)}\right)^{m-2}\left(x \delta^{\prime}(t)\left(1-\frac{x}{\delta(t)}\right)-(n-1)\right)
\end{aligned}
$$


Teorema 2.2.2 Para o perfil polinomial (2.6) a seguinte igualdade é satisfeita

$$
E_{L}=\frac{m}{\delta(t)^{3}}\left(\frac{(m-1)^{2} m}{2 m-3}+\frac{\delta(t) \delta^{\prime}(t)\left(-m(2 m+1)+\delta(t) \delta^{\prime}(t)\right)}{4 m^{2}-1}\right)
$$

Demonstração Substituindo $u$ pela aproximação (2.6)

$$
\begin{aligned}
& E_{L}=\int_{0}^{\delta(t)}\left(\frac{\partial u}{\partial t}-\frac{\partial^{2} u}{\partial x^{2}}\right)^{2} d x \\
& =\int_{0}^{\delta(t)}\left(\frac{\partial}{\partial t}\left(1-\frac{x}{\delta(t)}\right)^{m}-\frac{\partial^{2}}{\partial x^{2}}\left(1-\frac{x}{\delta(t)}\right)^{m}\right)^{2} d x \\
& =\int_{0}^{\delta(t)}\left(\frac{m x \delta^{\prime}(t)}{\delta(t)^{2}}\left(1-\frac{x}{\delta(t)}\right)^{m-1}-\frac{m(m-1)}{\delta(t)^{2}}\left(1-\frac{x}{\delta(t)}\right)^{m-2}\right)^{2} d x \\
& =\int_{0}^{\delta(t)} \frac{m^{2} x^{2} \delta^{\prime}(t)^{2}}{\delta(t)^{4}}\left(1-\frac{x}{\delta(t)}\right)^{2 m-2}-\frac{2 x m^{2}(m-1) \delta^{\prime}(t)}{\delta(t)^{4}}\left(1-\frac{x}{\delta(t)}\right)^{2 m-3} \\
& +\frac{m^{2}(m-1)^{2}}{\delta(t)^{4}}\left(1-\frac{x}{\delta(t)}\right)^{2 m-4} d x \\
& =\frac{m^{2} \delta^{\prime}(t)^{2}}{\delta(t)^{4}} \int_{0}^{\delta(t)} x^{2}\left(1-\frac{x}{\delta(t)}\right)^{2 m-2} d x-\frac{2 m^{2}(m-1) \delta^{\prime}(t)}{\delta(t)^{4}} \int_{0}^{\delta(t)} x\left(1-\frac{x}{\delta(t)}\right)^{2 m-3} d x \\
& +\frac{m^{2}(m-1)^{2}}{\delta(t)^{4}} \int_{0}^{\delta(t)}\left(1-\frac{x}{\delta(t)}\right)^{2 m-4} d x \\
& =\left.\frac{m^{2} \delta^{\prime}(t)^{2}}{\delta(t)^{4}}\left(\frac{\left(1-\frac{x}{\delta(t)}\right)^{2 m} \delta(t)^{2}\left(m(2 m-1) x^{2}+(2 m-1) x \delta(t)+\delta(t)^{2}\right)}{m(2 m-1)(2 m+1)(x-\delta(t))}\right)\right|_{0} ^{\delta(t)} \\
& -\left.\frac{2 m^{2}(m-1) \delta^{\prime}(t)}{\delta(t)^{4}}\left(-\frac{\left(1-\frac{x}{\delta(t)}\right)^{2 m} \delta(t)^{3}(2(m-1) x+\delta(t))}{2(m-1)(2 m-1)(x-\delta(t))^{2}}\right)\right|_{0} ^{\delta(t)} \\
& +\left.\frac{m^{2}(m-1)^{2}}{\delta(t)^{4}}\left(\frac{\left(1-\frac{x}{\delta(t)}\right)^{2 m} \delta(t)^{4}}{(2 m-3)(x-\delta(t))^{3}}\right)\right|_{0} ^{\delta(t)} \\
& =\frac{m^{2} \delta^{\prime}(t)^{2}}{\delta(t)^{4}} \frac{\delta(t)^{4}}{m(2 m-1)(2 m+1) \delta(t)}-\frac{2 m^{2}(m-1) \delta^{\prime}(t)}{\delta(t)^{4}} \frac{\delta(t)^{4}}{2(m-1)(2 m-1) \delta(t)^{2}} \\
& -\frac{m^{2}(m-1)^{2}}{\delta(t)^{4}} \frac{\delta(t)^{4}}{(2 m-3) \delta(t)^{3}}
\end{aligned}
$$

Tem-se, portanto, que

$$
\begin{aligned}
E_{L} & =\int_{0}^{\delta(t)}\left(\frac{\partial u}{\partial t}-\frac{\partial^{2} u}{\partial x^{2}}\right)^{2} d x \\
& =\frac{m \delta^{\prime}(t)^{2}}{(2 m-1)(2 m+1) \delta(t)}-\frac{m^{2} \delta^{\prime}(t)}{(2 m-1) \delta(t)^{2}}+\frac{(m-1)^{2} m^{2}}{(2 m-3) \delta(t)^{3}} \\
& =\frac{m}{\delta(t)^{3}}\left(\frac{(m-1)^{2} m}{2 m-3}+\frac{\delta(t) \delta^{\prime}(t)\left(-m(2 m+1)+\delta(t) \delta^{\prime}(t)\right)}{4 m^{2}-1}\right)
\end{aligned}
$$

$E_{L}$ depende de $m$ e de $\delta(t)$ 
Corolário 2.2.3 Para o método HBIM, em que $\delta(t)=\sqrt{2 m(m+1) t}$, tem-se que

$$
E_{L}=\frac{\left(2 m^{4}-7 m^{3}+6 m^{2}+2 m-1\right)}{4(m+1)^{2}(2 m-3)(2 m-1)(2 m+1)} \sqrt{2 m(m+1)} t^{-3 / 2}:=e_{L}(m) t^{-3 / 2}
$$

Demonstração Basta substituir $\delta(t)=\sqrt{2 m(m+1) t}$ na expressão do teorema (2.2.2).

Para o caso $m=2, \delta(t)=\sqrt{12 t}$. A solução é

$$
u(x, t)= \begin{cases}\left(1-\frac{x}{\delta(t)}\right)^{2} & \text { se } 0 \leq x \leq \delta(t) \\ 0 & \text { c.c. }\end{cases}
$$

A estimativa de energia nesse caso é

$$
E_{L}(t)=\frac{1}{30 \sqrt{3}} t^{-\frac{3}{2}}
$$

Para o caso $m=3, \delta(t)=\sqrt{24 t}$. A solução é

$$
u(x, t)= \begin{cases}\left(1-\frac{x}{\delta(t)}\right)^{3} & \text { se } 0 \leq x \leq \delta(t) \\ 0 & \text { c.c. }\end{cases}
$$

A estimativa de energia nesse caso é

$$
E_{L}(t)=\frac{5}{28 \sqrt{3}} t^{-\frac{3}{2}}
$$

As expressões da energia do teorema (2.2.2) e de seu corolário realçam alguns dos problemas inerentes à aplicação de métodos de balanceamento integral. A partir da proposição (2.2.1), para $x=0$,

$$
f(0, t)=-\frac{m(m-1)}{\delta^{2}}
$$

Para $u$ satisfazer a condição de contorno do problema $(f(0, t)=1)$, é necessário $m>1$. A expressão (2.10) entretanto mostra em $x=0$ que $f(0, t)$ nunca se anulará. Em $x=\delta$, tem-se

$$
f(\delta, t)=\lim _{x \rightarrow \delta} f(x, t)=-\frac{m(m-1)}{\delta^{2}} \lim _{x \rightarrow \delta}\left(1-\frac{x}{\delta}\right)^{m-2}
$$

A equação do calor só pode ser satisfeita em $x=\delta$ (ou seja $f(\delta, t)=0$ ) quando $m>2$. Para $m<2, f(\delta, t) \rightarrow \infty$ e para $m=2, f(\delta, t)=-2 / \delta^{2}$.

Na figura 2.5, no gráfico de $e_{L}(m)$ é possível observar que não há valor de $m$ de modo que $E_{L}$ seja 0. O trabalho de Mitchell e Myers (2012) mostra que para uma aproximação naturalmente se espera algum tipo de erro, mas o preocupante é que $E_{L}$ depende do tempo e, mais especificamente, à medida que $t \rightarrow 0, E_{L} \rightarrow \infty$, por mais precisa que a solução pareça. Portanto, enquanto $E_{L}$ pode ser útil para representar erros relativos (ou seja, para determinado tempo), é possível estudar $E_{L}$ para diferentes valores de $m$ a fim de determinar qual deles garante uma aproximação mais precisa. Essa medida, contudo, não é um parâmetro apropriado para quantificar o erro. No capítulo 3 será apresentada a técnica de imobilização de fronteira, em que a medida de erro apresentará um comportamento menos irreal que o de $E_{L}$. 


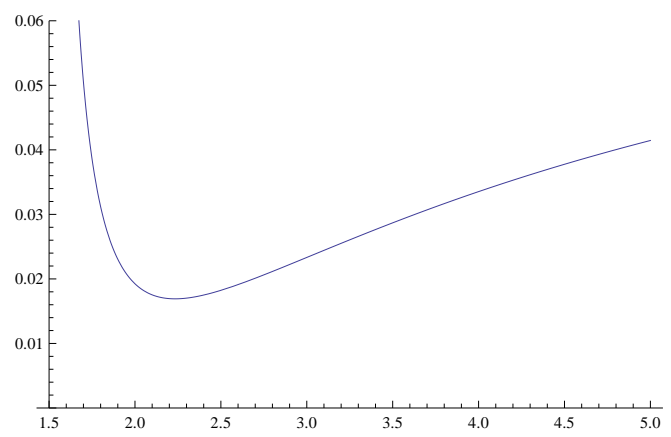

Figura 2.5: Gráfico de $e_{L}(m)$ para $m \in(1.5,5]$

\subsubsection{O Método de Balanceamento Integral Refinado}

O trabalho de Sadoun et al. (2006) nota que grande parte do erro real de aproximações de solução para problemas de Stefan vem da equação integral possuir uma derivada da aproximação. Assim, uma segunda integração é realizada, de modo que a equação integral contenha apenas informação da aproximação, e não de sua derivada. Utilizando-se do mesmo perfil aproximado (2.6) tem-se:

$$
\begin{aligned}
\int_{0}^{\delta}\left(\int_{0}^{x} \frac{\partial u}{\partial t}(\xi, t) d \xi\right) d x & =\int_{0}^{\delta}\left(\int_{0}^{x} \frac{\partial^{2} u}{\partial x^{2}}(\xi, t) d \xi\right) d x \\
& =\int_{0}^{\delta}\left(\frac{\partial u}{\partial x}-\left.\frac{\partial u}{\partial x}\right|_{x=0}\right) d x \\
& =\int_{0}^{\delta} \frac{\partial u}{\partial x} d x-\left(\left.x \frac{\partial u}{\partial x}\right|_{x=0}\right)_{0}^{\delta} \\
& =\left.u\right|_{x=\delta}-\left.u\right|_{x=0}-\left.\delta \frac{\partial u}{\partial x}\right|_{x=0}
\end{aligned}
$$

Por outro lado, o termo à esquerda da equação pode ser integrado por partes

$$
\begin{aligned}
\int_{0}^{\delta}\left(\int_{0}^{x} \frac{\partial u}{\partial t}(\xi, t) d \xi\right) d x & =\delta \int_{0}^{\delta} \frac{\partial u}{\partial t} d x-\int_{0}^{\delta} x \frac{\partial u}{\partial t} d x \\
& =\delta \int_{0}^{\delta} \frac{\partial^{2} u}{\partial x^{2}} d x-\int_{0}^{\delta} x \frac{\partial u}{\partial t} d x \\
& =\left.\delta \frac{\partial u}{\partial x}\right|_{x=\delta}-\left.\delta \frac{\partial u}{\partial x}\right|_{x=0}-\int_{0}^{\delta} x \frac{\partial u}{\partial t} d x \\
& =-\left.\delta \frac{\partial u}{\partial x}\right|_{x=0}-\int_{0}^{\delta} x \frac{\partial u}{\partial t} d x
\end{aligned}
$$

Combinando os últimos resultados, obtém-se

$$
\begin{aligned}
-\left.\delta \frac{\partial u}{\partial x}\right|_{x=0} & -\int_{0}^{\delta} x \frac{\partial u}{\partial t} d x=\left.u\right|_{x=\delta}-\left.u\right|_{x=0}-\left.\delta \frac{\partial u}{\partial x}\right|_{x=0} \\
- & \int_{0}^{\delta} x \frac{\partial u}{\partial t} d x=-\left.u\right|_{x=0} \\
\frac{d}{d t} \int_{0}^{\delta} x u d x-u(\delta(t), t) \delta^{\prime}(t) & =\left.u\right|_{x=0}
\end{aligned}
$$




$$
\frac{d}{d t} \int_{0}^{\delta} x u d x=\left.u\right|_{x=0}
$$

Substituindo o perfil aproximado (2.6) na equação obtida, tem-se:

$$
\begin{aligned}
\frac{d}{d t} \int_{0}^{\delta} x\left(1-\frac{x}{\delta}\right)^{m} d x & =\left.\left(1-\frac{x}{\delta}\right)^{m}\right|_{x=0} \\
\left.\frac{d}{d t}\left(-\delta \frac{\left(1-\frac{x}{\delta}\right)^{m+1}(x+m x+\delta)}{(m+1)(m+2)}\right)\right|_{0} ^{\delta} & =1 \\
\frac{d}{d t}\left(\frac{\delta^{2}}{(m+1)(m+2)}\right) & =1
\end{aligned}
$$

A família de soluções dessa equação diferencial sob um parâmetro $k$ é dada por:

$$
\delta(t)=\sqrt{(m+1)(m+2) t+k}
$$

Supondo-se $\delta(0)=0$, chega-se à solução

$$
\delta(t)=\sqrt{(m+1)(m+2)} \sqrt{t}
$$

E portanto a solução aproximada do problema 2.1 é dada por

$$
u(x, t)= \begin{cases}\left(1-\frac{x}{\delta(t)}\right)^{m} & \text { se } 0 \leq x \leq \delta(t) \\ 0 & \text { c.c. }\end{cases}
$$

\section{Comparação com a solução exata}

A análise da estimativa de energia proposta por Langford (1973) ainda exibe os mesmos inconvenientes para o método RIM.

Corolário 2.2.4 Para o método RIM, em que $\delta(t)=\sqrt{(m+1)(m+2) t}$, tem-se que

$$
E_{L}=\frac{10 m^{6}-39 m^{5}+34 m^{4}+27 m^{3}-20 m^{2}-12 m}{4(m+1)^{2}(m+2)^{2}(2 m-3)\left(4 m^{2}-1\right)} \sqrt{(m+1)(m+2)} t^{-3 / 2}=e_{L}(m) t^{-3 / 2}
$$

Demonstração Basta substituir $\delta(t)=\sqrt{(m+1)(m+2) t}$ na expressão do teorema (2.2.2).

Para $m=2$, tem-se

$$
E_{L}(t)=\frac{3}{20 \sqrt{6}} t^{-\frac{3}{2}}
$$

E para $m=3$,

$$
E_{L}(t)=\frac{2}{35 \sqrt{6}} t^{-\frac{3}{2}}
$$

\subsubsection{A escolha do expoente $m$}

Goodman (1958) trabalha primariamente com $m=2$. Mas existem diferentes abordagens para a escolha desse expoente. Uma delas é o chamado Combined Integral Method (CIM), onde se 


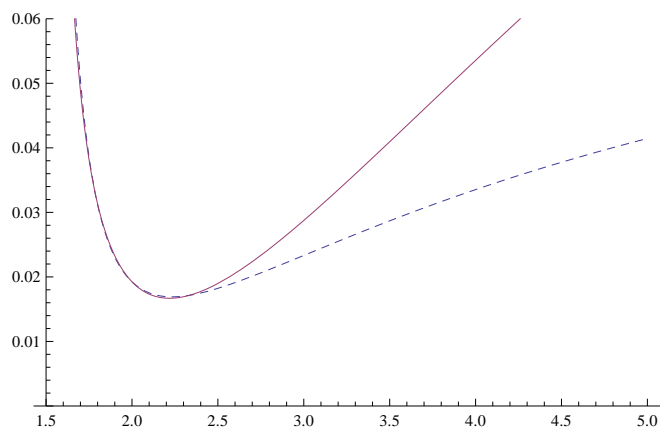

Figura 2.6: Gráfico de $e_{L}(m)$ para $m \in(1.5,5]$. A linha tracejada denota $e_{L}$ do método HBIM e a linha sólida, $e_{L}$ do método RIM

combinam HBIM e RIM. Imposta a condição de que a profundidade de penetração da temperatura seja igual ${ }^{1}$ tanto no método HBIM quanto no método RIM, obtém-se

$$
\begin{aligned}
\delta_{\mathrm{HBIM}}(t) & =\delta_{\mathrm{RIM}}(t) \\
\sqrt{2 m(m+1) t} & =\sqrt{(m+1)(m+2) t} \\
\sqrt{2 m} & =\sqrt{m+2} \\
m & =2
\end{aligned}
$$

O trabalho de Myers (2009) apresenta a técnica de minimização da função $E_{L}$ proposta por Langford (1973), cujas fórmulas são dadas pelos corolários 2.2.3 e 2.2.4. Os valores $m=2.2335$ e $m=2.2185$ são encontrados para os métodos HBIM e RIM respectivamente. Basta, nessas funções, minimizar a parte da função dependente de $m$, batizada de $e_{L}(m)$.

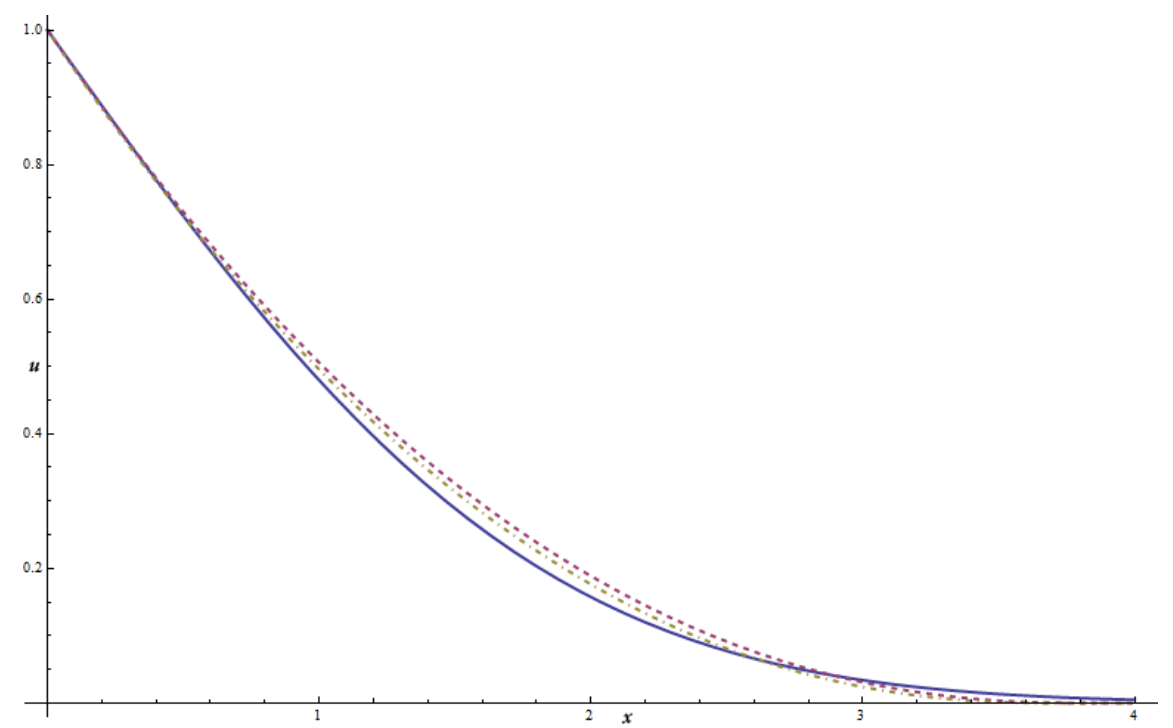

Figura 2.7: Gráfico da solução $u(x, t)$ em $t=1$. A solução exata é a linha sólida, a aproximação via HBIM e $m=2.2335$ é a linha tracejada, e a aproximação via RIM e $m=2.2185$ é a linha tracejada e pontilhada.

\footnotetext{
${ }^{1}$ Da mesma forma, é possível igualar as expressões de $\delta_{t}$ obtidas das equações diferenciais para $\delta$ de cada método, e novamente encontrar o mesmo resultado.
} 


\subsubsection{Refinamento usando subdivisões espaciais - o Método Nodal}

O trabalho de Bell e Abbas (1985) sugere um refinamento espacial que incorpora o balanceamento integral em uma abordagem numérica. Isto envolve a divisão da profundidade de penetração $\delta(t)$ em partes iguais e a solução para os valores de $u$ em cada subdivisão. Supondo-se que $x \in[0, \delta]$ seja subdividido em $n$ subintervalos de tamanho $\delta / n$, onde

$$
u\left(x_{i}, t\right)=u_{i}, x_{i}=\frac{i \delta(t)}{n}, i=0,1 \ldots, n
$$

as condições de fronteira geram $u_{0}=1$ e $u_{n}=0$. O balanceamento integral é determinado sobre cada subintervalo $\left[x_{i}, x_{i+1}\right]$ e então obtém-se

$$
\int_{x_{i}}^{x_{i+1}} \frac{\partial u}{\partial t} d x=\int_{x_{i}}^{x_{i+1}} \frac{\partial^{2} u}{\partial x^{2}} d x
$$

Como

$$
\frac{d}{d t} \int_{a(t)}^{b(t)} u(x, t) d x=\int_{a(t)}^{b(t)} \frac{\partial u}{\partial t}(x, t) d x+\frac{d b}{d t} u(b(t), t)-\frac{d a}{d t} u(a(t), t)
$$

e os valores $x_{i}$ e $x_{i+1}$ são funções de $t$ por possuírem $\delta(t)$, a integração resulta em

$$
\frac{d}{d t} \int_{x_{i}}^{x_{i+1}} u d x-x_{i+1}^{\prime} u_{i+1}+x_{i}^{\prime} u_{i}=\left.\frac{\partial u}{\partial x}\right|_{x=x_{i+1}}-\left.\frac{\partial u}{\partial x}\right|_{x=x_{i}}
$$

Incorporando as derivadas $x_{i}^{\prime}=\frac{i \delta^{\prime}}{n}$ e $x_{i+1}^{\prime}=\frac{(i+1) \delta^{\prime}}{n}$ dentro do sinal de derivação, obtém-se

$$
\frac{d}{d t}\left(\int_{x_{i}}^{x_{i+1}} u d x-\frac{(i+1) \delta}{n} u_{i+1}+\frac{i \delta}{n} u_{i}\right)=\left.\frac{\partial u}{\partial x}\right|_{x=x_{i+1}}-\left.\frac{\partial u}{\partial x}\right|_{x=x_{i}}
$$

Em Bell e Abbas (1985), para aproximar a temperatura em cada subintervalo $\left[x_{i}, x_{i+1}\right]$ são definidos $n$ perfis lineares da forma

$$
f_{i}(x)=u_{i}+\left(u_{i+1}-u_{i}\right) \frac{x-x_{i}}{x_{i+1}-x_{i}}, \quad i=0 . . n-1
$$


que automaticamente satisfazem $f_{i}\left(x_{i}\right)=u_{i}$ e $f_{i}\left(x_{i+1}\right)=u_{i+1}$. Substituindo $u$ por $f_{i}$ em $(2.14)$

$$
\begin{aligned}
\frac{d}{d t}\left(\int_{x_{i}}^{x_{i+1}} f_{i}(x) d x-\frac{(i+1) \delta}{n} u_{i+1}+\frac{i \delta}{n} u_{i}\right) & =f_{i}^{\prime}\left(x_{i+1}\right)-f_{i}^{\prime}\left(x_{i}\right) \\
\frac{d}{d t}\left(\int_{x_{i}}^{x_{i+1}} u_{i}+\left(u_{i+1}-u_{i}\right) \frac{x-x_{i}}{x_{i+1}-x_{i}} d x-\frac{(i+1) \delta}{n} u_{i+1}+\frac{i \delta}{n} u_{i}\right) & =f_{i}^{\prime}\left(x_{i+1}\right)-f_{i}^{\prime}\left(x_{i}\right) \\
\frac{d}{d t}\left(\left.\left(x u_{i}\right)\right|_{x_{i}} ^{x_{i+1}}+\left.\frac{u_{i+1}-u_{i}}{x_{i+1}-x_{i}}\left(\frac{x^{2}}{2}-x_{i} x\right)\right|_{x_{i}} ^{x_{i+1}}-\frac{(i+1) \delta}{n} u_{i+1}+\frac{i \delta}{n} u_{i}\right) & =f_{i}^{\prime}\left(x_{i+1}\right)-f_{i}^{\prime}\left(x_{i}\right) \\
\frac{d}{d t}\left(u_{i} \frac{\delta}{n}+\frac{u_{i+1}-u_{i}}{x_{i+1}-x_{i}}\left(\frac{x_{i+1}^{2}-x_{i}^{2}}{2}-x_{i} x_{i+1}+x_{i}^{2}\right)-\frac{(i+1) \delta}{n} u_{i+1}+\frac{i \delta}{n} u_{i}\right) & =f_{i}^{\prime}\left(x_{i+1}\right)-f_{i}^{\prime}\left(x_{i}\right) \\
\frac{d}{d t}\left(u_{i} \frac{\delta}{n}+\frac{u_{i+1}-u_{i}}{x_{i+1}-x_{i}}\left(x_{i+1}-x_{i}\right)\left(\frac{x_{i+1}+x_{i}}{2}-x_{i}\right)-\frac{(i+1) \delta}{n} u_{i+1}+\frac{i \delta}{n} u_{i}\right) & =f_{i}^{\prime}\left(x_{i+1}\right)-f_{i}^{\prime}\left(x_{i}\right) \\
\frac{d}{d t}\left(u_{i} \frac{\delta}{n}+\left(u_{i+1}-u_{i}\right) \frac{x_{i+1}-x_{i}}{2}-\frac{(i+1) \delta}{n} u_{i+1}+\frac{i \delta}{n} u_{i}\right) & =f_{i}^{\prime}\left(x_{i+1}\right)-f_{i}^{\prime}\left(x_{i}\right) \\
\frac{d}{d t}\left(u_{i} \frac{\delta}{n}+\left(u_{i+1}-u_{i}\right) \frac{\delta}{2 n}-\frac{(i+1) \delta}{n} u_{i+1}+\frac{i \delta}{n} u_{i}\right) & =f_{i}^{\prime}\left(x_{i+1}\right)-f_{i}^{\prime}\left(x_{i}\right) \\
\frac{d}{d t}\left(\frac{\delta}{2 n}\left(2 u_{i}+u_{i+1}-u_{i}-2(i+1) u_{i+1}+2 i u_{i}\right)\right) & =f_{i}^{\prime}\left(x_{i+1}\right)-f_{i}^{\prime}\left(x_{i}\right) \\
2 n & =f_{i}^{\prime}\left(x_{i+1}\right)-f_{i}^{\prime}\left(x_{i}\right)
\end{aligned}
$$

A escolha do perfil linear, entretanto, anula o lado direito da equação. A mudança no fluxo será representada pela variação descontínua em perfis adjacentes:

$$
\begin{gathered}
f_{i}^{\prime}\left(x_{i}\right)=\frac{u_{i+1}-u_{i}}{x_{i+1}-x_{i}}=\frac{u_{i+1}-u_{i}}{\delta / n}, \quad i=0 . . n-1 \\
f_{i}^{\prime}\left(x_{i+1}\right)=\frac{u_{i+2}-u_{i+1}}{x_{i+2}-x_{i+1}}=\frac{u_{i+2}-u_{i+1}}{\delta / n}, \quad i=0 . . n-1 \\
f_{i}^{\prime}\left(x_{i+1}\right)-f_{i}^{\prime}\left(x_{i}\right)=\frac{u_{i+2}-2 u_{i+1}+u_{i}}{\delta / n}, \quad i=0 . . n-1
\end{gathered}
$$

$\operatorname{com} f_{n}^{\prime}\left(x_{n+1}\right)=0$, já que $u_{x}(\delta, t)=0$. Assim,

$$
\delta \frac{d \delta}{d t}=-\frac{2 n^{2}}{2 i+1} \frac{u_{i+2}-2 u_{i+1}+u_{i}}{u_{i+1}-u_{i}}, \quad i=0 . . n-2
$$

Na equação (2.16), para $i=n-1$ tem-se:

$$
\begin{aligned}
-\frac{(2(n-1)+1)\left(u_{(n-1)+1}-u_{n-1}\right)}{2 n} \frac{d \delta}{d t} & =0-\frac{u_{(n-1)+1}-u_{n-1}}{\delta / n} \\
-\frac{(2 n-1)\left(u_{n}-u_{n-1}\right)}{2 n} \frac{d \delta}{d t} & =-\frac{u_{n}-u_{n-1}}{\delta / n} \\
\delta \frac{d \delta}{d t} & =\frac{2 n^{2}}{2 n-1}
\end{aligned}
$$


Dessa forma,

$$
\delta=\sqrt{\frac{4 n^{2} t}{2 n-1}}
$$

Combinando o resultado de (2.17) com (2.18) e eliminando o termo $\delta \frac{d \delta}{d t}$, tem-se para $i=0 . . n-2$

$$
\begin{aligned}
\frac{2 n^{2}}{2 n-1} & =-\frac{2 n^{2}}{2 i+1} \frac{u_{i+2}-2 u_{i+1}+u_{i}}{u_{i+1}-u_{i}} \\
-\left(u_{i+1}-u_{i}\right)(2 i+1) & =\left(u_{i+2}-2 u_{i+1}+u_{i}\right)(2 n-1) \\
-(2 n-2 i-2) u_{i} & =(2 n-1) u_{i+2}-(4 n-2 i-3) u_{i+1} \\
u_{i} & =-\frac{(2 n-1) u_{i+2}-(4 n-2 i-3) u_{i+1}}{(2 n-2 i-2)}
\end{aligned}
$$

Somando e subtraindo $u_{i+1}$ :

$$
\begin{aligned}
& u_{i}=-\frac{(2 n-1) u_{i+2}-(4 n-2 i-3) u_{i+1}}{(2 n-2 i-2)}+u_{i+1}-u_{i+1} \\
& u_{i}=u_{i+1}+\frac{-(2 n-2 i-2) u_{i+1}-(2 n-1) u_{i+2}+(4 n-2 i-3) u_{i+1}}{(2 n-2 i-2)} \\
& u_{i}=u_{i+1}+\frac{-(2 n-1) u_{i+2}+(2 n-1) u_{i+1}}{(2 n-2 i-2)} \\
& u_{i}=u_{i+1}-\frac{2 n-1}{2(n-i-1)}\left(u_{i+2}-u_{i+1}\right) \\
& u_{i}=u_{i+1}-\gamma_{i}\left(u_{i+2}-u_{i+1}\right)
\end{aligned}
$$

onde $\gamma_{i}:=\frac{2 n-1}{2(n-i-1)}$ e $i$ varia de 0 a $n-1$, formando um sistema de $n-1$ equações não-lineares em termos das temperaturas incógnitas $u_{i}$. Note que para $i=n-2$ e $i=n-3$, tem-se:

$$
\begin{aligned}
u_{n-2} & =u_{n-1}-\gamma_{i}\left(u_{n}-u_{n-1}\right) \\
& =u_{n-1}\left(1+\gamma_{n-2}\right) \\
u_{n-3} & =u_{n-2}-\gamma_{n-3}\left(u_{n-1}-u_{n-2}\right) \\
& =u_{n-1}\left(1+\gamma_{n-2}\right)-\gamma_{n-3}\left(u_{n-1}-u_{n-1}\left(1+\gamma_{n-2}\right)\right) \\
& =u_{n-1}\left(1+\gamma_{n-2}-\gamma_{n-3}+\gamma_{n-3}\left(1+\gamma_{n-2}\right)\right) \\
& =u_{n-1}\left(1+\gamma_{n-2}+\gamma_{n-3} \gamma_{n-2}\right)
\end{aligned}
$$

A seguinte fórmula de recorrência para $u_{i}$ é encontrada:

$$
\begin{aligned}
u_{i} & =u_{n-1}\left(1+\sum_{k=i}^{n-2} \prod_{j=k}^{n-2} \gamma_{j}\right) \\
& =u_{n-1} \sum_{k=i}^{n-1} \frac{\left(n-\frac{1}{2}\right)^{n-k-i}}{(n-k-1) !} \\
& =u_{n-1} \sum_{j=1}^{n-i} \frac{\left(n-\frac{1}{2}\right)^{j-1}}{(j-1) !}, \quad i=0,1, \ldots, n-2
\end{aligned}
$$


Como $u_{0}=1$ pela condição de contorno, obtém-se $u_{n-1}$, e a partir desse valor é possível encontrar os $n-2$ restantes através de substituição reversa. A solução aproximada é linear por partes e dada pela equação (2.15), onde

$$
u_{i}=\frac{\sum_{j=1}^{n-i}\left[\left(n-\frac{1}{2}\right)^{j-1} /(j-1) !\right]}{\sum_{j=1}^{n}\left[\left(n-\frac{1}{2}\right)^{j-1} /(j-1) !\right]}
$$

Essa técnica se assemelha com o método Variable Grid Space (VSG), pois também possui um tamanho de malha variável de acordo com o instante de tempo. Entretanto, o método VSG é um método de diferenças finitas, enquanto que o refinamento utilizando subdivisões espaciais é uma técnica semi-analítica, em que se propõem perfis de aproximações em cada subintervalo do domínio espacial. O trabalho de Bell e Abbas (1985) prova que a aproximação linear converge formalmente para a solução exata para valores grandes de $n$, usando propriedades da função Gama. Estudando o comportamento da temperatura nos pontos nodais da aproximação, prova-se a convergência pra solução exata. 


\section{Capítulo 3}

\section{O Problema Termal Revisitado}

\subsection{Condições de contorno}

No capítulo anterior foram introduzidos diferentes métodos para se aproximar soluções para um problema termal cuja condição de contorno era uma função constante. Este capítulo versa sobre a extensão dos métodos para problemas em que, por exemplo, a solução não é constante na fronteira, ou a condição se dá envolvendo o fluxo na fronteira. Também é introduzida neste capítulo a técnica conhecida por imobilização de fronteira.

\subsubsection{Fluxo constante na fronteira}

Ao se substituir a condição de fronteira no problema 2.1 por uma condição em que o fluxo $u_{x}(0, t)$ é constante e igual a -1 , o problema resulta em

$$
\begin{aligned}
\frac{\partial u}{\partial t}-\frac{\partial^{2} u}{\partial x^{2}} & =0, \quad 0<x<\infty \\
u(x, 0) & =0 \\
u_{x}(0, t) & =-1 \\
u & \rightarrow 0, \quad x \rightarrow \infty
\end{aligned}
$$

Esse problema ainda possui uma solução analítica, a saber

$$
u(x, t)=2 \sqrt{\frac{t}{\pi}} e^{-\frac{x^{2}}{4 t}}-x \operatorname{erfc} \frac{x}{2 \sqrt{t}}
$$

O perfil polinomial de aproximação assume a forma

$$
u=\frac{\delta(t)}{m}\left(1-\frac{x}{\delta(t)}\right)^{m}
$$

Para o método HBIM, a equação 2.7 se mantém. Resta substituir o novo perfil aproximado:

$$
\begin{aligned}
\frac{d}{d t} \int_{0}^{\delta} \frac{\delta}{m}\left(1-\frac{x}{\delta}\right)^{m} d x & =-\frac{\partial}{\partial x}\left(\left.\frac{\delta}{m}\left(1-\frac{x}{\delta}\right)^{m}\right|_{x=0}\right) \\
\frac{d}{d t}\left(\frac{\delta}{m} \int_{0}^{\delta}\left(1-\frac{x}{\delta}\right)^{m} d x\right) & =-\left.\frac{\delta}{m} \frac{\partial}{\partial x}\left(1-\frac{x}{\delta}\right)^{m}\right|_{x=0}
\end{aligned}
$$




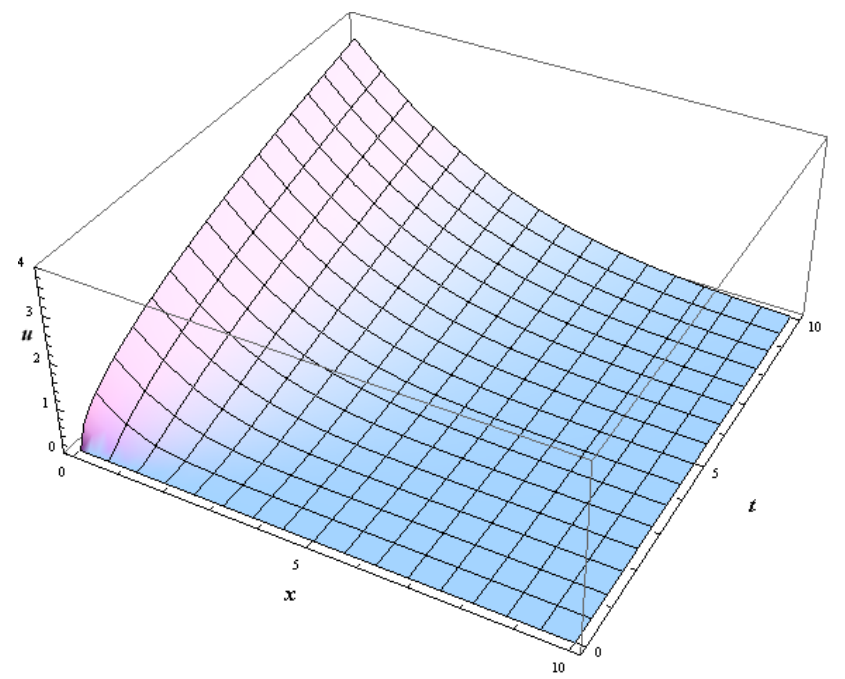

Figura 3.1: Gráfico da solução exata do problema 3.1

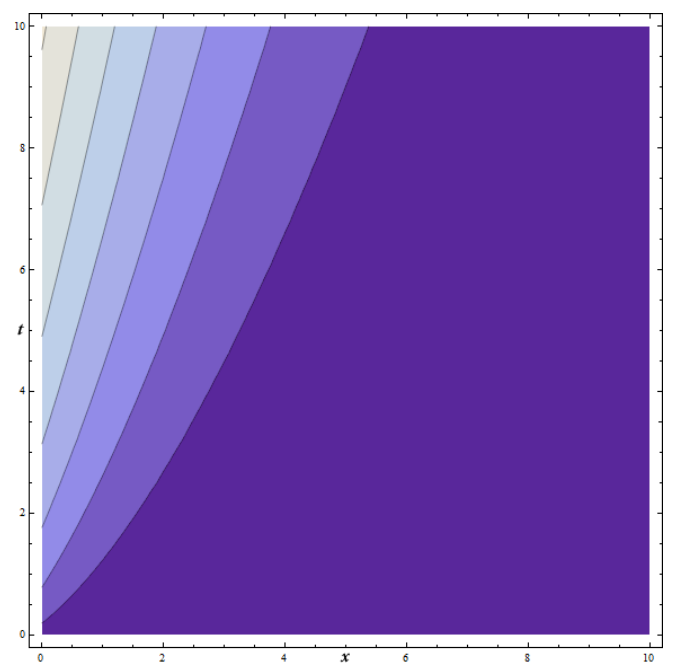

Figura 3.2: Curvas de nível da solução exata do problema 3.1

$$
\begin{aligned}
\frac{d}{d t}\left(\left.(x-\delta) \frac{\left(1-\frac{x}{\delta}\right)^{m+1}}{m+1}\right|_{0} ^{\delta} \frac{\delta}{m}\right) & =\left.\frac{\delta}{m} \frac{m}{\delta}\left(1-\frac{x}{\delta}\right)^{m-1}\right|_{x=0} \\
\frac{d}{d t}\left(\frac{\delta^{2}}{m(m+1)}\right) & =1
\end{aligned}
$$

cuja família de soluções a um parâmetro $k$ é $\sqrt{m t+m^{2} t+2 k}$. Como é imposto $\delta(0)=0$, a solução será $\delta(t)=\sqrt{m(m+1) t}$.

Para o método RIM, tem-se de maneira similar:

$$
\begin{aligned}
\frac{d}{d t} \int_{0}^{\delta} x u d x & =\left.u\right|_{x=0} \\
\frac{d}{d t} \int_{0}^{\delta} x \frac{\delta}{m}\left(1-\frac{x}{\delta}\right)^{m} d x & =\left.\frac{\delta}{m}\left(1-\frac{x}{\delta}\right)^{m}\right|_{x=0} \\
\left.\frac{d}{d t} \frac{\delta}{m}\left(\frac{\left(1-\frac{x}{\delta}\right)^{m}(x-\delta)(x+m x+\delta)}{(m+1)(m+2)}\right)\right|_{0} ^{\delta} & =\frac{\delta}{m}
\end{aligned}
$$




$$
\begin{aligned}
\frac{d}{d t} \frac{\delta}{m}\left(\frac{\delta^{2}}{(m+1)(m+2)}\right) & =\frac{\delta}{m} \\
\frac{d}{d t}\left(\frac{\delta^{3}}{(m+1)(m+2)}\right) & =\delta
\end{aligned}
$$

cuja solução é nada menos que $\delta(t)=\sqrt{\frac{2}{3}(m+1)(m+2) t}$.

Bem como foi discutido sobre a escolha de $m$ anteriormente, igualam-se $\delta_{\text {HBIM }}$ e $\delta_{\text {RIM }}$ de modo a se obter:

$$
\begin{aligned}
\delta_{\mathrm{HBIM}}(t) & =\delta_{\mathrm{RIM}}(t) \\
\sqrt{m(m+1) t} & =\sqrt{\frac{2}{3}(m+1)(m+2) t} \\
\sqrt{m} & =\sqrt{\frac{2}{3}(m+2)} \\
m & =4
\end{aligned}
$$

Caso a técnica de minimização do erro de Langford seja aplicada, Mitchell e Myers (2010b) encontram os valores $m=3.584$ para o HBIM e $m=3.822$ para o RIM.

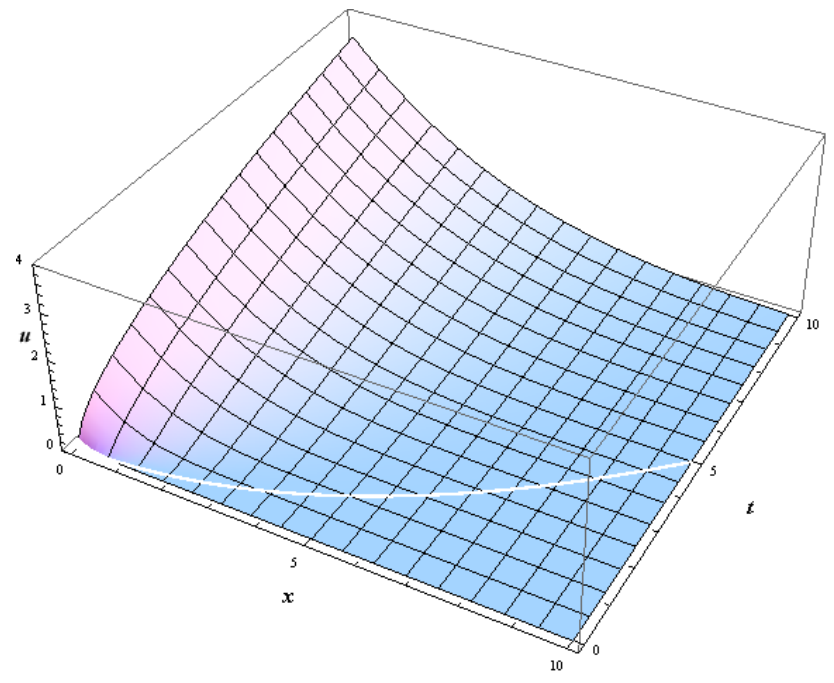

Figura 3.3: Aproximação via HBIM com fluxo constante na fronteira e $m=4$ obtido via CIM 


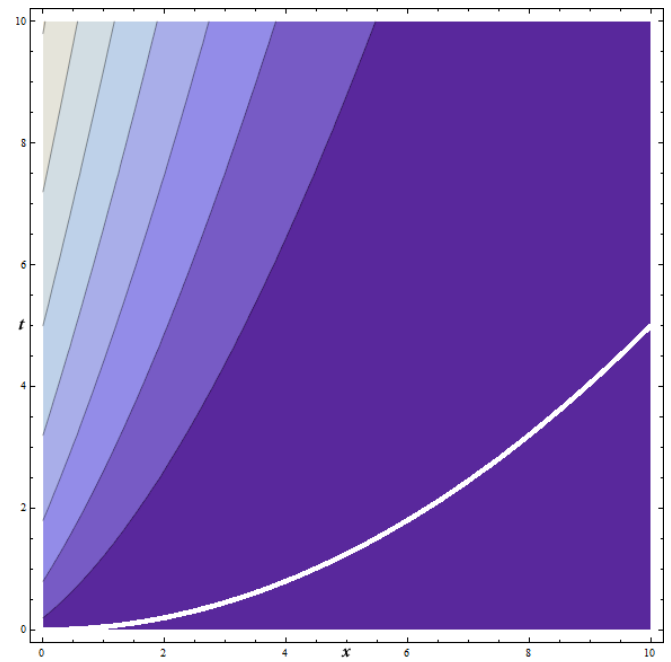

Figura 3.4: Curvas de nível da aproximação via HBIM com fluxo constante na fronteira e $m=4$

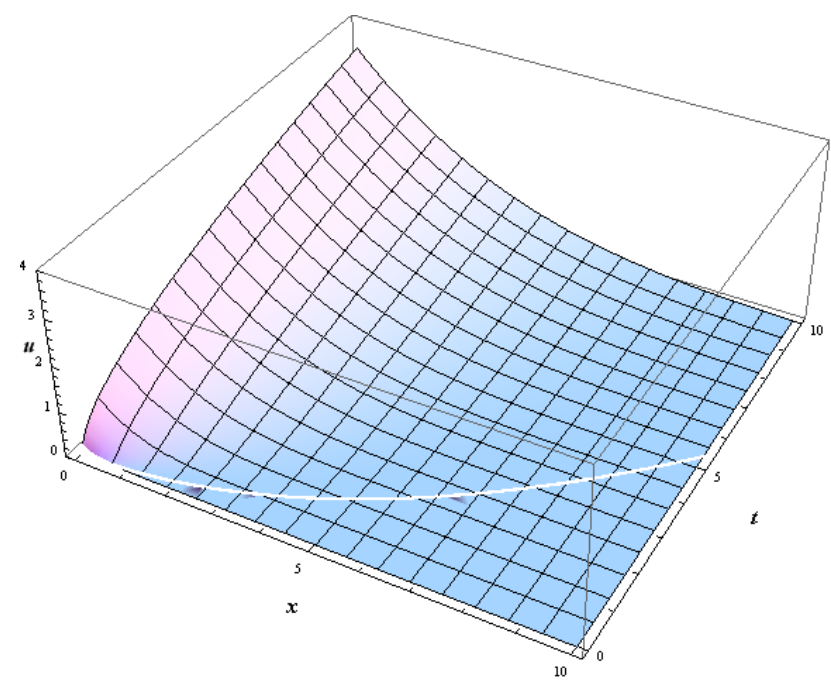

Figura 3.5: Aproximação via RIM com fluxo constante na fronteira e $m=3.822$ obtido via minimização do erro de Langford

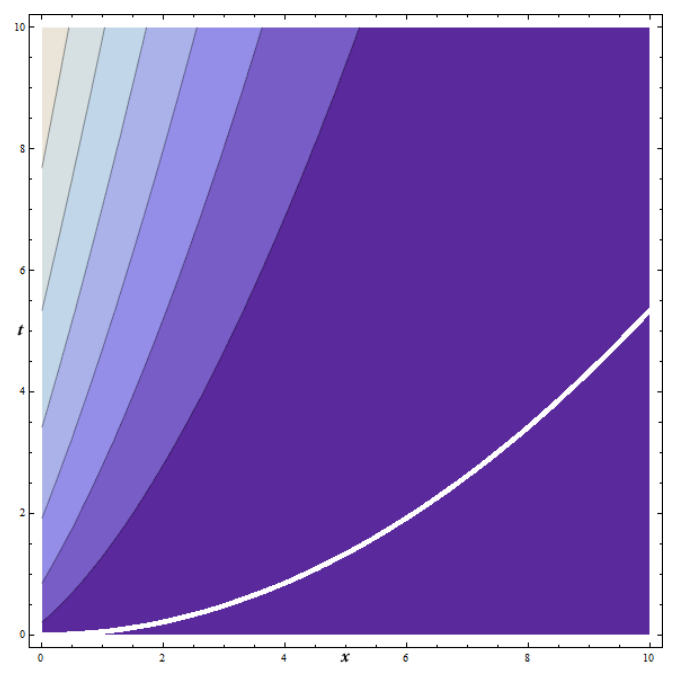

Figura 3.6: Curvas de nível da aproximação via RIM com fluxo constante na fronteira e $m=3.822$ 


\subsubsection{Condição de Resfriamento}

Substituindo agora a condição de fronteira no problema 2.1 por uma condição conhecida por condição de resfriamento $\left(u_{x}(0, t)=u(0, t)-1\right)$, obtém-se o seguinte problema

$$
\begin{aligned}
\frac{\partial u}{\partial t}-\frac{\partial^{2} u}{\partial x^{2}} & =0, \quad 0<x<\infty \\
u(x, 0) & =0 \\
u_{x}(0, t) & =u(0, t)-1 \\
u & \rightarrow 0, \quad x \rightarrow \infty
\end{aligned}
$$

Cuja solução analítica é :

$$
u(x, t)=\operatorname{erfc} \frac{x}{2 \sqrt{t}}-e^{x+t} \operatorname{erfc} \frac{x+2 t}{2 \sqrt{t}}
$$

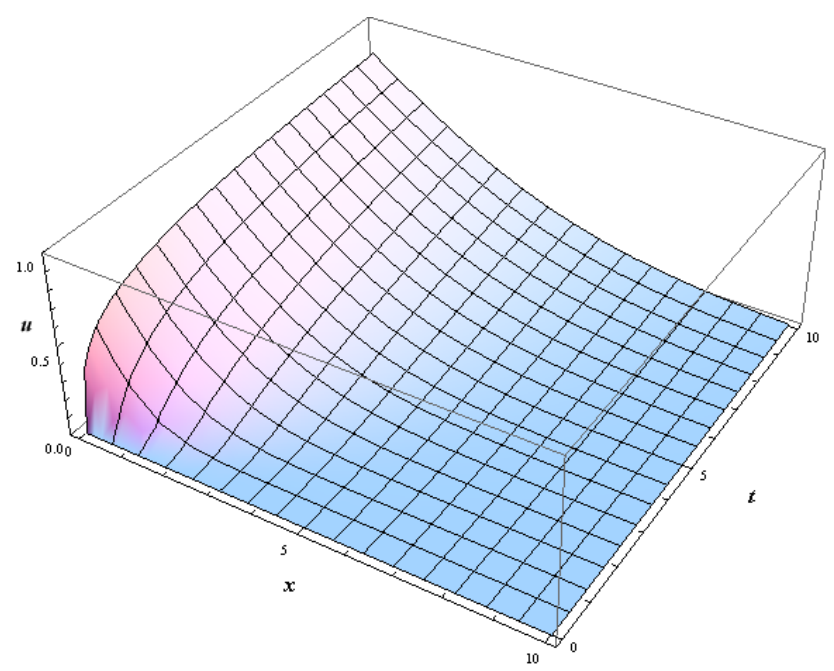

Figura 3.7: Gráfico da solução do problema 3.3

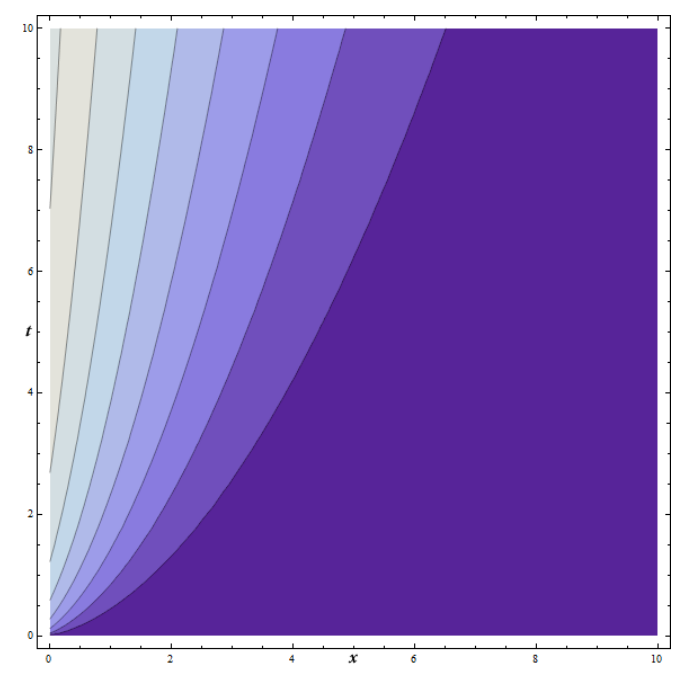

Figura 3.8: Curvas de nivel da solução do problema 3.3 
Se faz necessário o uso do seguinte perfil aproximado para a temperatura

$$
u=\frac{\delta(t)}{m+\delta(t)}\left(1-\frac{x}{\delta(t)}\right)^{m}
$$

Novamente substitui-se o perfil aproximado na equação do método HBIM

$$
\begin{aligned}
\frac{d}{d t} \int_{0}^{\delta} \frac{\delta}{m+\delta}\left(1-\frac{x}{\delta}\right)^{m} d x & =-\frac{\partial}{\partial x}\left(\left.\frac{\delta}{m+\delta}\left(1-\frac{x}{\delta}\right)^{m}\right|_{x=0}\right) \\
\frac{d}{d t}\left(\frac{\delta}{m+\delta} \int_{0}^{\delta}\left(1-\frac{x}{\delta}\right)^{m} d x\right) & =-\left.\frac{\delta}{m+\delta} \frac{\partial}{\partial x}\left(1-\frac{x}{\delta}\right)^{m}\right|_{x=0} \\
\frac{d}{d t}\left(\left.(x-\delta) \frac{\left(1-\frac{x}{\delta}\right)^{m+1}}{m+1}\right|_{0} ^{\delta} \frac{\delta}{m+\delta}\right) & =\left.\frac{\delta}{m+\delta} \frac{m}{\delta}\left(1-\frac{x}{\delta}\right)^{m-1}\right|_{x=0} \\
\frac{d}{d t}\left(\frac{\delta^{2}}{(m+1)(m+\delta)}\right) & =\frac{m}{m+\delta}
\end{aligned}
$$

Essa equação diferencial não tem solução analítica.

Para o método RIM, tem-se

$$
\begin{aligned}
\frac{d}{d t} \int_{0}^{\delta} x u d x & =\left.u\right|_{x=0} \\
\frac{d}{d t} \int_{0}^{\delta} x \frac{\delta}{m+\delta}\left(1-\frac{x}{\delta}\right)^{m} d x & =\left.\frac{\delta}{m+\delta}\left(1-\frac{x}{\delta}\right)^{m}\right|_{x=0} \\
\left.\frac{d}{d t} \frac{\delta}{m+\delta}\left(\frac{\left(1-\frac{x}{\delta}\right)^{m}(x-\delta)(x+m x+\delta)}{(m+1)(m+2)}\right)\right|_{0} ^{\delta} & =\frac{\delta}{m+\delta} \\
\frac{d}{d t} \frac{\delta}{m+\delta}\left(\frac{\delta^{2}}{(m+1)(m+2)}\right) & =\frac{\delta}{m+\delta} \\
\frac{d}{d t}\left(\frac{\delta^{3}}{(m+1)(m+2)(m+\delta)}\right) & =\frac{\delta}{m+\delta}
\end{aligned}
$$

que também não possui solução analítica. Assumindo $m$ constante e igualando a derivada $\delta^{\prime}$ das equações diferenciais para os métodos acima,

$$
\begin{aligned}
\frac{2 \delta \delta^{\prime}}{(m+1)(m+\delta)}-\frac{\delta^{2} \delta^{\prime}}{(m+1)(m+\delta)^{2}} & =\frac{m}{m+\delta} \\
\delta^{\prime} & =\frac{m(m+1)(m+\delta)}{\delta(2 m+\delta)} \\
\frac{3 \delta^{2} \delta^{\prime}}{(m+1)(m+2)(m+\delta)}-\frac{\delta^{3} \delta^{\prime}}{(m+1)(m+2)(m+\delta)^{2}} & =\frac{\delta}{m+\delta} \\
\delta^{\prime} & =\frac{(m+1)(m+2)(m+\delta)}{\delta(3 m+2 \delta)}
\end{aligned}
$$

E portanto

$$
\begin{aligned}
\frac{m(m+1)(m+\delta)}{\delta(2 m+\delta)} & =\frac{(m+1)(m+2)(m+\delta)}{\delta(3 m+2 \delta)} \\
\frac{(m+1)(m+\delta)((m-2) \delta+(m-4) m)}{\delta(2 m+\delta)(3 m+2 \delta)} & =0
\end{aligned}
$$


cujo resultado é

$$
m=\frac{4-\delta \pm \sqrt{16+\delta^{2}}}{2}
$$

E assim fica claro que assumir $m$ constante é incorreto e $m=m(\delta(t))=m(t)$. Nesse caso o problema se reduz a resolver numericamente em conjunto as duas equações diferenciais resultantes dos métodos RIM e HBIM para $m(t)$ e $\delta(t)$ (método CIM). As aproximações para as soluções das equações 3.7 e 3.12 são mostradas nas figuras 3.9 e 3.10. As condições iniciais para resolver essas equações são $\delta(0)=0$ e $m(0)=4$, uma vez que a condição de resfriamento no limite $t \rightarrow 0$ recai sobre a condição de fluxo constante na fronteira.

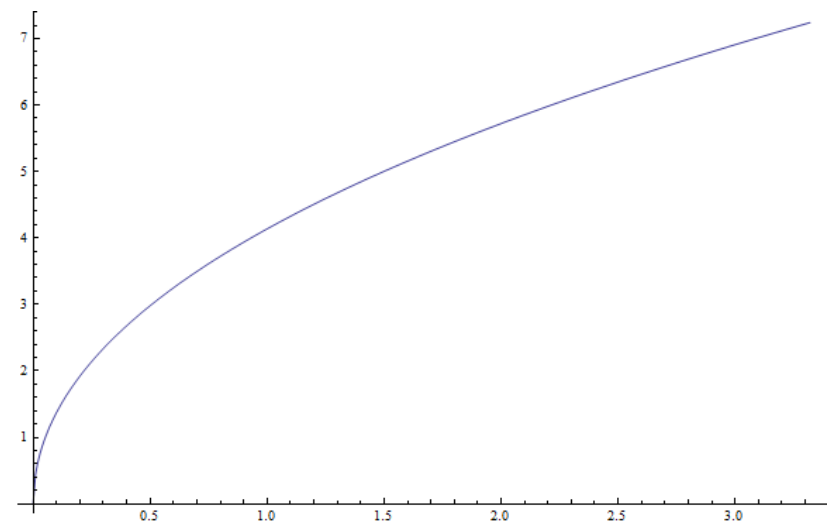

Figura 3.9: Gráfico de $\delta(t)$ para o problema termal com condição de resfriamento (obtido numericamente a partir do método CIM)

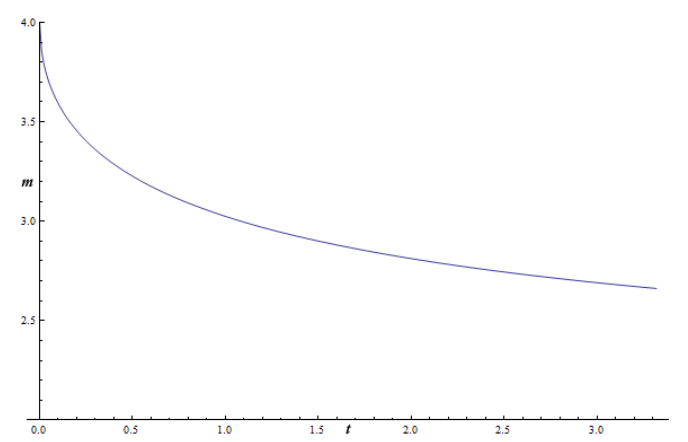

Figura 3.10: Curva do expoente $m$ dependente do tempo obtido numericamente a partir do método CIM para o problema termal com condição de resfriamento

A seguir, o gráfico das aproximações obtidas a partir de $\delta(t)$ e $m(t)$

Usando a técnica de minimização do erro de Langford, Myers (2009) também encontra que nesse caso $m$ é dependente do tempo. Por causa de $m=m(t)$, toda a fórmula de minimização requer um novo cálculo, uma vez que o termo $u_{t}$ envolveria derivadas em $m$. O resultado é um sistema de equações diferenciais não lineares para determinar os valores de $m(t)$ e $\delta(t)$. Uma sugestão feita a fim de se evitar este problema é substituir $m(t)$ por $m(0)$, pois o erro $E_{L}$ é máximo em $m=0$. 


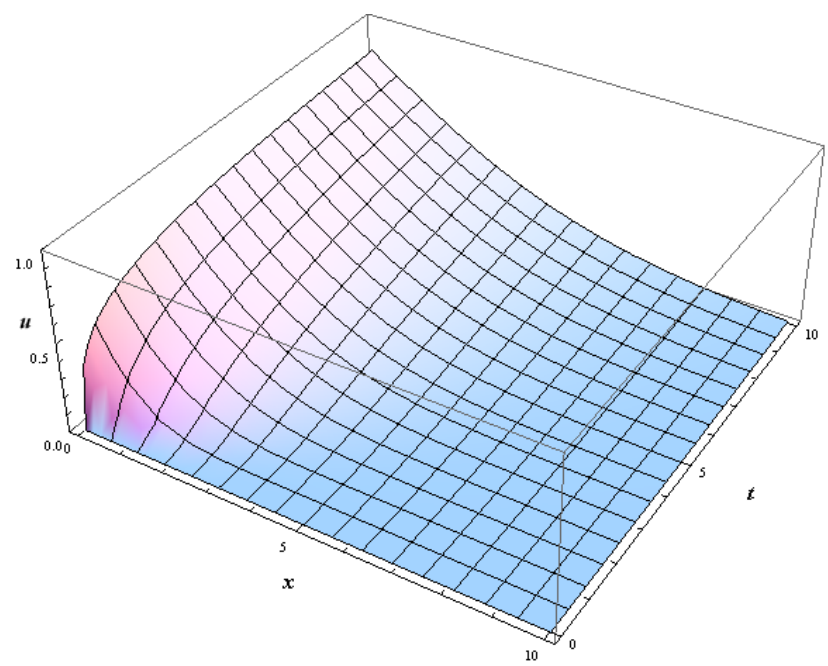

Figura 3.11: Gráfico da aproximação via CIM do problema 2.1 com condição de resfriamento

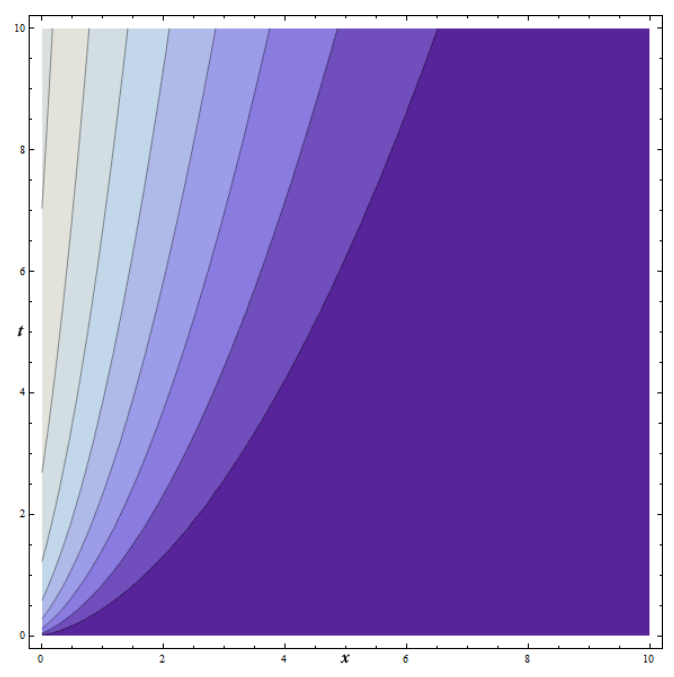

Figura 3.12: Curvas de nível da aproximação via CIM problema 2.1 com condição de resfriamento

\subsection{Imobilização de fronteira}

Para problemas termais, a técnica de imobilização de fronteira consiste basicamente em introduzir uma mudança de coordenadas

$$
\xi=\frac{x}{\delta(t)}
$$

que transforma o domínio móvel $0<x<\delta(t)$ e um domínio fixo $0<\xi<1$. Voltando ao problema 2.1, em que a condição de fronteira é constante e igual a 1 , e denotando $u(x, t)=U(\xi, t)$, tem-se

$$
\begin{aligned}
u(x, t) & =U(\xi(x, t), t) \\
u_{t}(x, t) & =\frac{\partial U}{\partial \xi}(\xi(x, t), t) \frac{\partial \xi}{\partial t}(x, t)+\frac{\partial U}{\partial t}(\xi(x, t), t) \\
u_{x}(x, t) & =\frac{\partial U}{\partial \xi}(\xi(x, t), t) \frac{\partial \xi}{\partial x}(x, t) \\
u_{x x}(x, t) & =\frac{\partial^{2} U}{\partial \xi}(\xi(x, t), t) \frac{\partial \xi}{\partial x}(x, t) \frac{\partial \xi}{\partial x}(x, t)+\frac{\partial U}{\partial \xi}(\xi(x, t), t) \frac{\partial^{2} \xi}{\partial x^{2}}(x, t) \\
u_{t}(x, t)-u_{x x}(x, t) & =\left(\frac{\partial U}{\partial \xi}(\xi(x, t), t) \frac{\partial \xi}{\partial t}(x, t)+\frac{\partial U}{\partial t}(\xi(x, t), t)\right)
\end{aligned}
$$




$$
-\left(\frac{\partial^{2} U}{\partial \xi^{2}}(\xi(x, t), t)\left(\frac{\partial \xi}{\partial x}(x, t)\right)^{2}+\frac{\partial U}{\partial \xi}(\xi(x, t), t) \frac{\partial^{2} \xi}{\partial x^{2}}(x, t)\right)
$$

Como $\xi(x, t)=\frac{x}{\delta(t)}$, tem-se que

$$
\begin{aligned}
& \frac{\partial \xi}{\partial x}(x, t)=\frac{1}{\delta(t)} \\
& \frac{\partial \xi}{\partial t}(x, t)=-\frac{x}{\delta(t)^{2}} \delta^{\prime}(t)=-\frac{\xi(x, t) \delta^{\prime}(t)}{\delta(t)}
\end{aligned}
$$

Logo,

$u_{t}(x, t)-u_{x x}(x, t)=\left(-\frac{\partial U}{\partial \xi}(\xi(x, t), t) \frac{\xi(x, t) \delta^{\prime}(t)}{\delta(t)}+\frac{\partial U}{\partial t}(\xi(x, t), t)\right)-\left(\frac{\partial^{2} U}{\partial \xi^{2}}(\xi(x, t), t)\left(\frac{1}{\delta(t)}\right)^{2}+0\right)$ $u_{t}(x, t)-u_{x x}(x, t)=U_{t}-\frac{\delta^{\prime} \xi}{\delta} U_{\xi}-\frac{1}{\delta^{2}} U_{\xi \xi}$

E, portanto, a equação do calor se torna

$$
\frac{\partial U}{\partial t}-\frac{\delta^{\prime}(t) \xi}{\delta(t)} \frac{\partial U}{\partial \xi}=\frac{1}{\delta(t)^{2}} \frac{\partial^{2} U}{\partial \xi^{2}}
$$

e as novas condições de contorno são

$$
U(0, t)=1, \quad U(1, t)=\frac{\partial U}{\partial \xi}(1, t)=0
$$

A aproximação polinomial 2.6 é agora $U=(1-\xi)^{m}$. Este perfil possui a característica de ser independente de $\delta$, ou seja, a solução tem a forma constante no tempo.

Análogo à definição da função $f$, tem-se

$$
\begin{aligned}
F(\xi, t) & =\delta(t)^{2} \frac{\partial U}{\partial t}-\delta(t) \delta^{\prime}(t) \xi \frac{\partial U}{\partial \xi}-\frac{\partial^{2} U}{\partial \xi^{2}} \\
& =-\delta(t) \delta^{\prime}(t) \xi \frac{\partial U}{\partial \xi}-\frac{\partial^{2} U}{\partial \xi^{2}}
\end{aligned}
$$

A formulação do método HBIM é substituída por

$$
\begin{aligned}
\int_{0}^{1} F(\xi, t) d \xi & =0 \\
\int_{0}^{1}-\delta(t) \delta^{\prime}(t) \xi \frac{\partial U}{\partial \xi}-\frac{\partial^{2} U}{\partial \xi^{2}} d \xi & =0 \\
\int_{0}^{1} \delta(t) \delta^{\prime}(t) m \xi(1-\xi)^{m-1}-m(m-1)(1-\xi)^{m-2} d \xi & =0 \\
-\left.\delta(t) \delta^{\prime}(t) m \frac{(1-x)^{m}(m x+1)}{m(m+1)}\right|_{0} ^{1}+\left.m(1-x)^{m-1}\right|_{0} ^{1} & =0 \\
\frac{\delta(t) \delta^{\prime}(t)}{m+1}-m & =0
\end{aligned}
$$

E obtém-se, novamente, a solução $\delta(t)=\sqrt{2 m(m+1) t}$. 
Para o método RIM,

$$
\begin{aligned}
\int_{0}^{1} \xi F(\xi, t) d \xi & =0 \\
-\left.\delta(t) \delta^{\prime}(t) m \frac{(1-x)^{m}\left(m^{2} x^{2}+m(x+2) x+2\right)}{m(m+1)(m+2)}\right|_{0} ^{1}+\left.(1-x)^{m-1}((m-1) x+1)\right|_{0} ^{1} & =0 \\
\frac{2 \delta(t) \delta^{\prime}(t)}{(m+1)(m+2)}-1 & =0 \\
2 \delta(t) \delta^{\prime}(t) & =(m+1)(m+2)
\end{aligned}
$$

Portanto, $\delta(t)=\sqrt{(m+1)(m+2) t}$.

A técnica de imobilização de fronteira apresentada até o momento não introduziu novidades com relação a soluções do problema. Entretanto, uma melhor estimativa de erro pode ser obtida da equação de mínimos quadrados da equação imobilizada:

$$
E_{M}=\int_{0}^{1} F(\xi, t)^{2} d \xi=\int_{0}^{1}\left(\delta(t) \delta^{\prime}(t) \xi \frac{\partial U}{\partial \xi}+\frac{\partial^{2} U}{\partial \xi^{2}}\right)^{2} d \xi
$$

Teorema 3.2.1 Para o novo perfil polinomial $U=(1-\xi)^{m}$, a seguinte igualdade é satisfeita

$$
E_{M}=\frac{\left(\delta \delta^{\prime}\right)^{2} m}{(2 m-1)(2 m+1)}-\frac{\delta \delta^{\prime} m^{2}}{(2 m-1)}+\frac{m^{2}(m-1)^{2}}{(2 m-3)}
$$

\section{Demonstração}

$$
\begin{aligned}
E_{M} & =\int_{0}^{1}\left(\delta \delta^{\prime} \xi u_{\xi}+u_{\xi \xi}\right)^{2} d \xi \\
& =\int_{0}^{1}\left(\delta \delta^{\prime}\right)^{2} \xi^{2} u_{\xi}^{2}+2 \delta \delta^{\prime} \xi \mathrm{u}_{\xi} u_{\xi \xi}+u_{\xi \xi}{ }^{2} d \xi \\
& =\left(\delta \delta^{\prime}\right)^{2} \int_{0}^{1} \xi^{2}\left(m(1-\xi)^{m-1}\right)^{2} d \xi-2 \delta \delta^{\prime} \int_{0}^{1} \xi m(1-\xi)^{m-1} m(m-1)(1-\xi)^{m-2} d \xi \\
& +\int_{0}^{1}\left(m(m-1)(1-\xi)^{m-2}\right)^{2} d \xi \\
& =\left(\delta \delta^{\prime}\right)^{2} m^{2} \int_{0}^{1} \xi^{2}(1-\xi)^{2 m-2} d \xi-2 \delta \delta^{\prime} m^{2}(m-1) \int_{0}^{1} \xi(1-\xi)^{2 m-3} d \xi \\
& +m^{2}(m-1)^{2} \int_{0}^{1}(1-\xi)^{2 m-4} d \xi \\
& =\left.\left(\delta \delta^{\prime}\right)^{2} m^{2}\left(-\frac{(1-\xi)^{2 m-1}\left(m(2 m-1) \xi^{2}+(2 m-1) \xi+1\right)}{m\left(4 m^{2}-1\right)}\right)\right|_{0} ^{1} \\
& -\left.2 \delta \delta^{\prime} m^{2}(m-1)\left(-\frac{(1-\xi)^{2(m-1)}(2(m-1) \xi+1)}{2(m-1)(2 m-1)}\right)\right|_{0} ^{1}+\left.m^{2}(m-1)^{2}\left(-\frac{(1-\xi)^{2 m-3}}{2 m-3}\right)\right|_{0} ^{1} \\
& =\left(\delta \delta^{\prime}\right)^{2} m^{2}\left(\frac{1}{m(2 m-1)(2 m+1)}\right)-2 \delta \delta^{\prime} m^{2}(m-1)\left(\frac{\delta \delta^{\prime} m^{2}}{2(m-1)(2 m-1)}+\frac{m^{2}(m-1)^{2}}{(2 m-3)}\right. \\
& \left.=\frac{\left(\delta \delta^{\prime}\right)^{2} m}{(2 m-1)(2 m+1)}-\frac{m^{2}(m-1)^{2}\left(\frac{1}{2 m-3}\right)}{(2 m-1)}\right)
\end{aligned}
$$


Corolário 3.2.2 Para o método HBIM,

$$
E_{M}=\frac{m^{2}\left(2 m^{4}-7 m^{3}+6 m^{2}+2 m-1\right)}{(2 m-1)(2 m+1)(2 m-3)}
$$

Demonstração Basta notar que $\delta \delta^{\prime}=m(m+1)$ no HBIM.

Corolário 3.2.3 Para o método RIM,

$$
E_{M}=\frac{m(m-1)\left(10 m^{4}-29 m^{3}+5 m^{2}+32 m+12\right)}{4(2 m-3)(2 m-1)(2 m+1)}
$$

Demonstração Basta notar que $\delta \delta^{\prime}=\frac{(m+1)(m+2)}{2}$ no RIM.

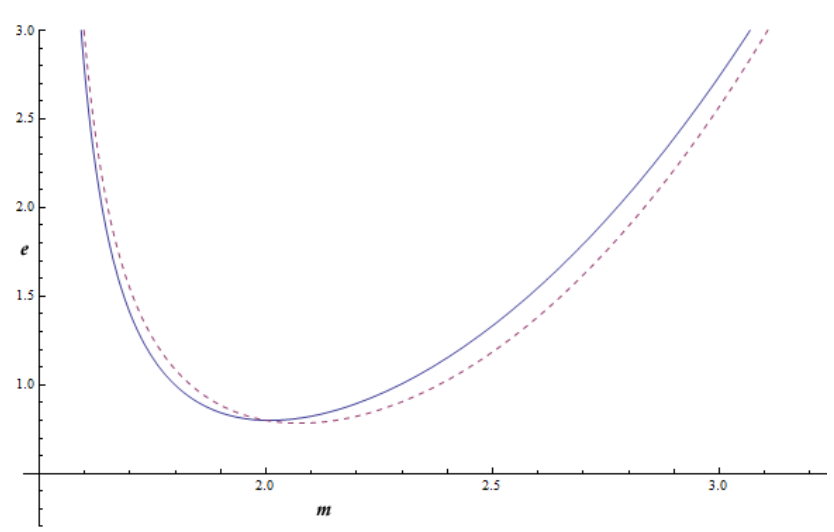

Figura 3.13: Gráfico de $E_{M}$ para HBIM e RIM. Os pontos de mínimo são $m=2.008$ para o HBIM e $m=2.074$ para o $R I M$

É importante notar que para esse problema foi definido um erro que é independente do tempo e, em particular, não singular em $t=0$. É relacionado ao erro definido por Langford por $E_{M} \sim \delta^{3} E_{L}$.

\subsubsection{Fluxo constante na fronteira}

Revendo o problema com fluxo constante na fronteira

$$
\begin{aligned}
\frac{\partial u}{\partial t}-\frac{\partial^{2} u}{\partial x^{2}} & =0, \quad 0<x<\infty \\
u(x, 0) & =0 \\
u_{x}(0, t) & =-1 \\
u & \rightarrow 0, \quad x \rightarrow \infty
\end{aligned}
$$

mas agora utilizando-se da técnica de imobilização, o perfil de aproximação 3.2 assume a forma

$$
U(\xi, t)=\frac{1}{m}(1-\xi)^{m}
$$


independente de $\delta$. Aqui é feito uma reescala de $u=\delta U$, que não foi necessária na condição de fronteira constante pois $u(0, t)$ era 1 . A equação do calor é dada por

$$
\frac{\partial}{\partial t}(\delta U)-\delta_{t} \xi \frac{\partial U}{\partial \xi}=\frac{1}{\delta} \frac{\partial^{2} U}{\partial \xi^{2}}
$$

e como $U_{t}=0$, isso se reduz a

$$
\delta \delta_{t} U-\delta \delta_{t} \xi \frac{\partial U}{\partial \xi}=\frac{\partial^{2} U}{\partial \xi^{2}}
$$

A função $F(\xi, t)$ é portanto dada por

$$
F(\xi, t)=\delta \delta_{t} U-\delta \delta_{t} \xi \frac{\partial U}{\partial \xi}-\frac{\partial^{2} U}{\partial \xi^{2}}
$$

Em Mitchell e Myers (2012), são exibidas as formulações de HBIM e RIM para este caso, bem como os valores $E_{M}$. A formulação HBIM exibe $\delta=\sqrt{m(m+1) t}$ e

$$
E_{M}=\frac{(n-1)\left(2 n^{3}-13 n^{2}+19 n+10\right)}{4(2 n-1)(2 n+1)(2 n-3)}
$$

Similarmente, o RIM exibe $\delta=\sqrt{\frac{2}{3}(n+1)(n+2) t}$ e

$$
E_{M}=\frac{10 n^{6}-79 n^{5}+160 n^{4}+38 n^{3}-167 n^{2}-40 n+24}{9 n^{2}(2 n-1)(2 n+1)(2 n-3)}
$$

Em ambos os casos, $E_{M}$ é independente de $t$. A expressão correspondente utilizando-se do método de Langford possui $E_{L} \sim t^{-1 / 2}$, como no problema de temperatura constante: ela explode à medida que $t \rightarrow 0$.

\subsubsection{Condição de Resfriamento}

Fazendo $\xi=\frac{x}{\delta}$ e $u(x, t)=\delta U(\xi, t)$, obtém-se a seguinte aproximação polinomial

$$
U=\frac{1}{m+\delta}(1-\xi)^{m}
$$

Novamente, conclui-se que $m$ deve depender do tempo $t$. Neste caso, ao integrar a equação 3.16, as formulações do HBIM e do RIM são

$$
\begin{gathered}
\text { HBIM: } \delta \delta_{t} \int_{0}^{1} U d \xi+\delta \frac{d}{d t} \int_{0}^{1} \delta U d \xi=-\left.\frac{\partial U}{\partial \xi}\right|_{\xi=0} \\
\text { RIM: } 2 \delta \delta_{t} \int_{0}^{1} \xi U d \xi+\delta \frac{d}{d t} \int_{0}^{1} \delta \xi U d \xi=\left.U\right|_{\xi=0}
\end{gathered}
$$

Definindo $F(\xi, t)$ como anteriormente, o erro $E_{M}=\int_{0}^{1} F(\xi, t)^{2} d \xi$ é

$$
\begin{aligned}
E_{M}=\int_{0}^{1} & {\left[\frac{m \delta^{2} \delta_{t}}{(m+\delta)^{2}}(1-\xi)^{m}+\frac{\delta^{3} m_{t}}{m+\delta}(1-\xi)^{m} \ln (1-\xi)-\frac{\delta^{3} m_{t}}{(m+\delta)^{2}}(1-\xi)^{m}\right.} \\
& \left.+\frac{m \delta^{2} \delta_{t}}{m+\delta}(1-\xi)^{m-1}-\frac{m(m-1) \delta}{m+\delta}(1-\xi)^{m-2}\right]^{2} d \xi
\end{aligned}
$$


Essa expressão, apesar de parecer extremamente complicada, pode ser integrada analiticamente, como nota Mitchell e Myers (2012). Como nesse caso $m=m(t)$ e $E_{M}=E_{M}(m)$, conclui-se que $E_{M}=E_{M}(t)$. Mas não há singularidade em $t=0\left(E_{M}(0)=0\right)$. Para evitar as dificuldades de se analisar um expoente dependente do tempo, no estudo de Myers (2010), a solução foi calculada com $m(t)=m(0)$. Esse valor foi escolhido pois, dada a natureza singular de $E_{L}$, o maior valor do erro ocorre em $t=0$. 


\subsection{Imobilização de Fronteira e Subdivisões Espaciais}

O trabalho de Mitchell (2011) propõe um aperfeiçoamento na técnica de Bell e Abbas (1985) apresentada na seção 2.2.4 ao utilizar perfis de aproximações locais não necessariamente lineares. O uso de perfis quadráticos ou cúbicos proporciona melhoras significativas com relação ao perfil linear. É necessário apenas combinar o número apropriado de derivadas para determinar os coeficientes extras desconhecidos. Na técnica de Bell e Abbas, havia a questão de o lado direito da equação 2.16 se anular. Isso não ocorre em perfis de ordens maiores. Entretanto, como os $u_{i}$ 's eram supostos constantes, aquele método não podia ser aplicado a outros tipos de condições de fronteira, como fluxo constante e condições de resfriamento.

Um perfil quadrático a ser estudado por exemplo seria

$$
f_{i}(x)=u_{i}+\alpha_{i} \frac{\left(x-x_{i}\right)}{x_{i+1}-x_{i}}+\beta_{i} \frac{\left(x-x_{i}\right)^{2}}{\left(x_{i+1}-x_{i}\right)^{2}}, \quad i=0, \ldots, n-1
$$

em que os coeficientes $\alpha_{i}$ e $\beta_{i}$ são provenientes da exigência $f_{i}\left(x_{i+1}\right)=u_{i+1}$ e $f_{i}^{\prime}\left(x_{i+1}\right)=f_{i+1}^{\prime}\left(x_{i+1}\right)$. Se a condição de fronteira em $x=0$ é a condição de fluxo constante $u_{x}=-1$, então isso significa que $f^{\prime}\left(x_{0}\right)=-1$. Usando o perfil quadrático acima e a definição de $x_{i}$, tem-se $\alpha_{0}=-\frac{\delta}{n}$; Portanto, $\alpha_{0}$ é dependente do tempo, o que não pode ser tratado com o método de Bell e Abbas.

Através da imobilização de fronteira e do perfil generalizado ${ }^{1}$

$$
\xi=\frac{x}{\delta(t)}, \quad u(x, t)=\phi(t) U(\xi, t)
$$

as equações do problema termal se tornam:

$$
\begin{gathered}
\phi U_{\xi \xi}=\delta\left(\delta \phi_{t} U+\delta \phi U_{t}-\xi \delta_{t} \phi U_{\xi}\right) \\
U=U_{\xi}=0, \quad \text { em } \xi=1
\end{gathered}
$$

com as condições de fronteira exibidas na tabela 3.1. Nesta tabela também são apresentados os perfis aproximados e as equações do problema após a escolha de $\phi$. Essa escolha garante que sejam recuperadas as condições conhecidas $U(0, t)=1$ (fronteira com temperatura constante), $U_{\xi}(0, t)=$ -1 (fluxo constante na fronteira), $U_{\xi}(0, t)=\delta U(0, t)-1$ (condição de resfriamento).

\begin{tabular}{|c|c|c|c|c|c|}
\hline Condição & em $\xi=0$ & $\phi$ & após escolha de $\phi$ & Equação & Perfil \\
\hline temp. constante & $U=\frac{1}{\phi}$ & 1 & $U=1$ & $U_{\xi \xi}=\delta^{2} U_{t}-\xi \delta \delta_{t} U_{\xi}$ & $(1-\xi)^{m}$ \\
fluxo constante & $U=-\frac{\delta}{\phi}$ & $\delta$ & $U_{\xi}=-1$ & $U_{\xi \xi}=\delta \delta_{t} U+\delta^{2} U_{t}-\xi \delta \delta_{t} U_{\xi}$ & $\frac{1}{m}(1-\xi)^{m}$ \\
resfriamento & $U=\delta\left(U-\frac{1}{\phi}\right)$ & $\delta$ & $U_{\xi}=\delta U-1$ & $U_{\xi \xi}=\delta \delta_{t} U+\delta^{2} U_{t}-\xi \delta \delta_{t} U_{\xi}$ & $\frac{1}{m+\delta}(1-\xi)^{m}$ \\
\hline
\end{tabular}

Tabela 3.1: Imobilização de Fronteira para diferentes condições

\footnotetext{
${ }^{1}$ A função $\phi$ é introduzida para representar de maneira genérica todos os perfis de aproximação apresentados. Para cada perfil $\phi$ assume um valor distinto
} 


\subsubsection{Condição de temperatura constante na fronteira}

Aplicando o método de balanceamento integral nodal para o problema transformado, o domínio de $\xi$ é fixo, e portanto, pode-se subdividir $\xi$ em partes iguais e independentes de $\delta$. Fazendo

$$
U\left(\xi_{i}\right)=U_{i}, \quad \xi_{i}=\frac{i}{n}, \quad i=0, \ldots, n
$$

as condições de fronteira se tornam $U_{0}=1$ e $U_{n}=0$. Neste caso a aproximação polinomial é $(1-\xi)^{m}$ e a equação diferencial se torna apenas (uma vez que $U_{t}=0$ ):

$$
U_{\xi \xi}=-\xi \delta \delta_{t} U_{\xi}
$$

Note que

$$
\int_{\xi_{i}}^{\xi_{i+1}} \xi \frac{\partial U}{\partial \xi} d \xi=\left.\xi U\right|_{\xi_{i}} ^{\xi_{i+1}}-\int_{\xi_{i}}^{\xi_{i+1}} U d \xi
$$

Portanto a equação de balanceamento integral se torna

$$
-\delta \delta_{t}\left(\xi_{i+1} U_{i+1}-\xi_{i} U_{i}-\int_{\xi_{i}}^{\xi_{i+1}} U d \xi\right)=\left.\frac{\partial U}{\partial \xi}\right|_{\xi=\xi_{i+1}}-\left.\frac{\partial U}{\partial \xi}\right|_{\xi=\xi_{i}}
$$

Como mencionado, é possível utilizar-se de um perfil polinomial quadrático fazendo as correspondências das derivadas em cada ponto nodal. Contudo, isso é essencialmente uma interpolação de spline, e splines quadráticas são conhecidas por apresentarem efeitos oscilatórios severos. Mitchell (2011) lida com essa questão utilizando-se de splines cúbicas, mas também faz uma análise para splines quadráticas. Como splines cúbicas exigem correspondências nos pontos nodais não só das primeiras, quanto das segundas derivadas, é necessário mais trabalho que para o perfil quadrático. Mas garante-se uma aproximação suave para a derivada da temperatura, bem como para a própria temperatura.

Em cada subintervalo $\left[\xi_{i}, \xi_{i+1}\right], U$ é aproximado pelo seguinte perfil cúbico

$$
f_{i}(\xi)=U_{i}+\alpha_{i} \frac{\left(\xi-\xi_{i}\right)}{\left(\xi_{i+1}-\xi_{i}\right)}+\beta_{i} \frac{\left(\xi-\xi_{i}\right)^{2}}{\left(\xi_{i+1}-\xi_{i}\right)^{2}}+\gamma_{i} \frac{\left(\xi-\xi_{i}\right)^{3}}{\left(\xi_{i+1}-\xi_{i}\right)^{3}}
$$

Os coeficientes $\alpha_{i}, \beta_{i}, \gamma_{i}$ são determinados como parte do processo de solução ao corresponder os $f_{i}$ 's, suas derivadas e segundas derivadas nos pontos nodais. Essa forma já garante que $f_{i}\left(\xi_{i}\right)=U_{i}$. Para garantir $f_{i}\left(\xi_{i+1}\right)=U_{i+1}$, deve-se ter

$$
\alpha_{i}+\beta_{i}+\gamma_{i}=U_{i+1}-U_{i}
$$

para $i=0,1, \ldots, n-1$.

Correspondendo a derivada e a segunda derivada, isto é, $f_{i}^{\prime}\left(\xi_{i+1}\right)=f_{i+1}^{\prime}\left(\xi_{i+1}\right)$ e $f_{i}^{\prime \prime}\left(\xi_{i+1}\right)=$ $f_{i+1}^{\prime \prime}\left(\xi_{i+1}\right)$ tem-se

$$
\alpha_{i}+2 \beta_{i}+3 \gamma_{i}=\alpha_{i+1}, \quad 2 \beta_{i}+6 \gamma_{i}=2 \beta_{i+1}
$$

para $i=0,1, \ldots, n-2$.

Como $U_{\xi}(1, t)=0$, segue que $f_{n-1}^{\prime}\left(\xi_{n}\right)=0$. Como também $U_{\xi \xi}(1, t)=0$, segue também que 
$f_{n-1}^{\prime \prime}\left(\xi_{n}\right)=0$. E portanto, pelas últimas expressões

$$
\alpha_{n-1}+2 \beta_{n-1}+3 \gamma_{n-1}=0, \quad 2 \beta_{n-1}+6 \gamma_{n-1}=0
$$

Combinando as duas últimas equações com a equação $n-1$ de 3.17 , utilizando-se do fato que $U_{n}=0$, obtém-se:

$$
\alpha_{n-1}=-3 U_{n-1}, \quad \beta_{n-1}=3 U_{n-1}, \quad \gamma_{n-1}=-U_{n-1}
$$

Agora, ao substituir o perfil cúbico aproximado na equação de balanceamento integral, notando que

$$
\begin{aligned}
\int_{\xi_{i}}^{\xi_{i+1}} U d \xi & =\int_{\xi_{i}}^{\xi_{i+1}} U_{i}+\alpha_{i} \frac{\left(\xi-\xi_{i}\right)}{\left(\xi_{i+1}-\xi_{i}\right)}+\beta_{i} \frac{\left(\xi-\xi_{i}\right)^{2}}{\left(\xi_{i+1}-\xi_{i}\right)^{2}}+\gamma_{i} \frac{\left(\xi-\xi_{i}\right)^{3}}{\left(\xi_{i+1}-\xi_{i}\right)^{3}} d \xi \\
& =U_{i}\left(\xi_{i+1}-\xi_{i}\right)+\alpha_{i} \frac{\xi_{i+1}-\xi_{i}}{2}+\beta_{i} \frac{\xi_{i+1}-\xi_{i}}{3}+\gamma_{i} \frac{\xi_{i+1}-\xi_{i}}{4} \\
& =\left(\xi_{i+1}-\xi_{i}\right)\left(U_{i}+\frac{\alpha_{i}}{2}+\frac{\beta_{i}}{3}+\frac{\gamma_{i}}{4}\right)
\end{aligned}
$$

que

$$
\begin{aligned}
\left.\frac{\partial U}{\partial \xi}\right|_{\xi=\xi_{i+1}} & =\left.\frac{\partial}{\partial \xi}\left(U_{i}+\alpha_{i} \frac{\left(\xi-\xi_{i}\right)}{\left(\xi_{i+1}-\xi_{i}\right)}+\beta_{i} \frac{\left(\xi-\xi_{i}\right)^{2}}{\left(\xi_{i+1}-\xi_{i}\right)^{2}}+\gamma_{i} \frac{\left(\xi-\xi_{i}\right)^{3}}{\left(\xi_{i+1}-\xi_{i}\right)^{3}}\right)\right|_{\xi=\xi_{i+1}} \\
& =\left.\left(\frac{\alpha_{i}}{\left(\xi_{i+1}-\xi_{i}\right)}+\beta_{i} \frac{2\left(\xi-\xi_{i}\right)}{\left(\xi_{i+1}-\xi_{i}\right)^{2}}+\gamma_{i} \frac{3\left(\xi-\xi_{i}\right)^{2}}{\left(\xi_{i+1}-\xi_{i}\right)^{3}}\right)\right|_{\xi=\xi_{i+1}} \\
& =\frac{\alpha_{i}}{\left(\xi_{i+1}-\xi_{i}\right)}+\beta_{i} \frac{2\left(\xi_{i+1}-\xi_{i}\right)}{\left(\xi_{i+1}-\xi_{i}\right)^{2}}+\gamma_{i} \frac{3\left(\xi_{i+1}-\xi_{i}\right)^{2}}{\left(\xi_{i+1}-\xi_{i}\right)^{3}} \\
& =\frac{\alpha_{i}}{\left(\xi_{i+1}-\xi_{i}\right)}+\frac{2 \beta_{i}}{\left(\xi_{i+1}-\xi_{i}\right)}+\frac{3 \gamma_{i}}{\left(\xi_{i+1}-\xi_{i}\right)} \\
& =\frac{\alpha_{i}+2 \beta_{i}+3 \gamma_{i}}{\left(\xi_{i+1}-\xi_{i}\right)}
\end{aligned}
$$

e que

$$
\begin{aligned}
\left.\frac{\partial U}{\partial \xi}\right|_{\xi=\xi_{i}} & =\left.\frac{\partial}{\partial \xi}\left(U_{i}+\alpha_{i} \frac{\left(\xi-\xi_{i}\right)}{\left(\xi_{i+1}-\xi_{i}\right)}+\beta_{i} \frac{\left(\xi-\xi_{i}\right)^{2}}{\left(\xi_{i+1}-\xi_{i}\right)^{2}}+\gamma_{i} \frac{\left(\xi-\xi_{i}\right)^{3}}{\left(\xi_{i+1}-\xi_{i}\right)^{3}}\right)\right|_{\xi=\xi_{i}} \\
& =\left.\left(\frac{\alpha_{i}}{\left(\xi_{i+1}-\xi_{i}\right)}+\beta_{i} \frac{2\left(\xi-\xi_{i}\right)}{\left(\xi_{i+1}-\xi_{i}\right)^{2}}+\gamma_{i} \frac{3\left(\xi-\xi_{i}\right)^{2}}{\left(\xi_{i+1}-\xi_{i}\right)^{3}}\right)\right|_{\xi=\xi_{i}} \\
& =\frac{\alpha_{i}}{\left(\xi_{i+1}-\xi_{i}\right)}
\end{aligned}
$$

e portanto

$$
\begin{aligned}
-\delta \delta_{t}\left(\xi_{i+1} U_{i+1}-\xi_{i} U_{i}-\int_{\xi_{i}}^{\xi_{i+1}} U d \xi\right) & =\left.\frac{\partial U}{\partial \xi}\right|_{\xi=\xi_{i+1}}-\left.\frac{\partial U}{\partial \xi}\right|_{\xi=\xi_{i}} \\
-\delta \delta_{t}\left(\xi_{i+1} U_{i+1}-\xi_{i} U_{i}-\left(\xi_{i+1}-\xi_{i}\right)\left(U_{i}+\frac{\alpha_{i}}{2}+\frac{\beta_{i}}{3}+\frac{\gamma_{i}}{4}\right)\right) & =\frac{\alpha_{i}+2 \beta_{i}+3 \gamma_{i}}{\left(\xi_{i+1}-\xi_{i}\right)}-\frac{\alpha_{i}}{\left(\xi_{i+1}-\xi_{i}\right)} \\
-\delta \delta_{t}\left(\xi_{i+1}\left(U_{i+1}-U_{i}\right)-\xi_{i+1}\left(\frac{\alpha_{i}}{2}+\frac{\beta_{i}}{3}+\frac{\gamma_{i}}{4}\right)+\xi_{i}\left(\frac{\alpha_{i}}{2}+\frac{\beta_{i}}{3}+\frac{\gamma_{i}}{4}\right)\right) & =\frac{2 \beta_{i}+3 \gamma_{i}}{\left(\xi_{i+1}-\xi_{i}\right)} \\
-\delta \delta_{t}\left(\frac{(i+1)}{n}\left(U_{i+1}-U_{i}\right)-\frac{1}{n}\left(\frac{\alpha_{i}}{2}+\frac{\beta_{i}}{3}+\frac{\gamma_{i}}{4}\right)\right) & =\frac{2 \beta_{i}+3 \gamma_{i}}{1 / n}
\end{aligned}
$$




$$
-\delta \delta_{t}\left((i+1)\left(U_{i+1}-U_{i}\right)-\left(\frac{\alpha_{i}}{2}+\frac{\beta_{i}}{3}+\frac{\gamma_{i}}{4}\right)\right)=n^{2}\left(2 \beta_{i}+3 \gamma_{i}\right)
$$

Logo,

$$
\delta \delta_{t}=\frac{n^{2}\left(2 \beta_{i}+3 \gamma_{i}\right)}{\frac{\alpha_{i}}{2}+\frac{\beta_{i}}{3}+\frac{\gamma_{i}}{4}-(i+1)\left(U_{i+1}-U_{i}\right)}
$$

Substituindo $i=n-1$ e usando as expressões de $\alpha_{n-1}, \beta_{n-1}$ e $\gamma_{n-1}$, obtém-se

$$
\delta \delta_{t}=\frac{12 n^{2}}{4 n-3}
$$

E eliminando $\delta \delta_{t}$ ao igualar as duas últimas expressões, obtém-se

$$
\begin{aligned}
\frac{n^{2}\left(2 \beta_{i}+3 \gamma_{i}\right)}{(i+1)\left(U_{i+1}-U_{i}\right)-\left(\frac{\alpha_{i}}{2}+\frac{\beta_{i}}{3}+\frac{\gamma_{i}}{4}\right)} & =\frac{12 n^{2}}{4 n-3} \\
12\left((i+1)\left(U_{i+1}-U_{i}\right)-\left(\frac{\alpha_{i}}{2}+\frac{\beta_{i}}{3}+\frac{\gamma_{i}}{4}\right)\right) & =(4 n-3)\left(2 \beta_{i}+3 \gamma_{i}\right) \\
12(i+1)\left(U_{i+1}-U_{i}\right)-6 \alpha_{i}-4 \beta_{i}-3 \gamma_{i} & =2(4 n-3) \beta_{i}+3(4 n-3) \gamma_{i} \\
6 \alpha_{i}+(10-8 n) \beta_{i}+(12-12 n) \gamma_{i}-12(i+1)\left(U_{i+1}-U_{i}\right) & =0
\end{aligned}
$$

Logo,

$$
3 \alpha_{i}+(5-4 n) \beta_{i}+6(1-n) \gamma_{i}-6(i+1)\left(U_{i+1}-U_{i}\right)=0
$$

As equações 3.17, 3.18 e 3.19 permitem encontrar as $4 n-1$ incógnitas $\alpha_{i}, \beta_{i}, \gamma_{i}$ e $U_{i}$; e $U_{0}$ já é conhecido. Isto pode ser obtido ao escrever o sistema $A x=b$, onde

$$
x=\left[U_{0}, \alpha_{0}, \beta_{0}, \gamma_{0}, U_{1}, \alpha_{1}, \beta_{1}, \gamma_{1}, \ldots, U_{n-1}, \alpha_{n-1}, \beta_{n-1}, \gamma_{n-1}\right]^{T}
$$

a matriz $A$ contém os coeficientes das equações $3.17,3.18$ e 3.19 e o vetor-coluna $b$ tem a última entrada igual a 1 e as restantes 0 (para dar a condição de fronteira $U_{0}=1$ ).

Por exemplo, para $n=4$, as equações 3.17 ficam

$$
\begin{array}{r}
U_{0}-U_{1}+\alpha_{0}+\beta_{0}+\gamma_{0}=0 \\
U_{1}-U_{2}+\alpha_{1}+\beta_{1}+\gamma_{1}=0 \\
U_{2}-U_{3}+\alpha_{2}+\beta_{2}+\gamma_{2}=0 \\
U_{3}+\alpha_{3}+\beta_{3}+\gamma_{3}=0
\end{array}
$$

as equações 3.18 ficam

$$
\begin{aligned}
\alpha_{0}-\alpha_{1}+2 \beta_{0}+3 \gamma_{0} & =0 \\
\alpha_{1}-\alpha_{2}+2 \beta_{1}+3 \gamma_{1} & =0 \\
\alpha_{2}-\alpha_{3}+2 \beta_{2}+3 \gamma_{2} & =0 \\
2 \beta_{0}-2 \beta_{1}+6 \gamma_{0} & =0 \\
2 \beta_{1}-2 \beta_{2}+6 \gamma_{1} & =0 \\
2 \beta_{2}-2 \beta_{3}+6 \gamma_{2} & =0
\end{aligned}
$$


as equações 3.19 ficam

$$
\begin{array}{r}
6 U_{0}-6 U_{1}+3 \alpha_{0}-11 \beta_{0}-18 \gamma_{0}=0 \\
12 U_{1}-12 U_{2}+3 \alpha_{1}-11 \beta_{1}-18 \gamma_{1}=0 \\
18 U_{2}-18 U_{3}+3 \alpha_{2}-11 \beta_{2}-18 \gamma_{2}=0
\end{array}
$$

e as equações para $n-1$ ficam

$$
\begin{aligned}
3 U_{3}+\alpha_{3} & =0 \\
-3 U_{3}+\beta_{3} & =0 \\
U_{3}+\gamma_{3} & =0
\end{aligned}
$$

Unindo todas essas equações com $U_{0}=1$ na forma $A x=b$, tem-se o sistema esparso:

$$
\left(\begin{array}{cccccccccccccccc}
1 & 1 & 1 & 1 & -1 & 0 & 0 & 0 & 0 & 0 & 0 & 0 & 0 & 0 & 0 & 0 \\
0 & 0 & 0 & 0 & 1 & 1 & 1 & 1 & -1 & 0 & 0 & 0 & 0 & 0 & 0 & 0 \\
0 & 0 & 0 & 0 & 0 & 0 & 0 & 0 & 1 & 1 & 1 & 1 & -1 & 0 & 0 & 0 \\
0 & 0 & 0 & 0 & 0 & 0 & 0 & 0 & 0 & 0 & 0 & 0 & 1 & 1 & 1 & 1 \\
0 & 1 & 2 & 3 & 0 & -1 & 0 & 0 & 0 & 0 & 0 & 0 & 0 & 0 & 0 & 0 \\
0 & 0 & 0 & 0 & 0 & 1 & 2 & 3 & 0 & -1 & 0 & 0 & 0 & 0 & 0 & 0 \\
0 & 0 & 0 & 0 & 0 & 0 & 0 & 0 & 0 & 1 & 2 & 3 & 0 & -1 & 0 & 0 \\
0 & 0 & 2 & 6 & 0 & 0 & -2 & 0 & 0 & 0 & 0 & 0 & 0 & 0 & 0 & 0 \\
0 & 0 & 0 & 0 & 0 & 0 & 2 & 6 & 0 & 0 & -2 & 0 & 0 & 0 & 0 & 0 \\
0 & 0 & 0 & 0 & 0 & 0 & 0 & 0 & 0 & 0 & 2 & 6 & 0 & 0 & -2 & 0 \\
6 & 3 & -11 & -18 & -6 & 0 & 0 & 0 & 0 & 0 & 0 & 0 & 0 & 0 & 0 & 0 \\
0 & 0 & 0 & 0 & 12 & 3 & -11 & -18 & -12 & 0 & 0 & 0 & 0 & 0 & 0 & 0 \\
0 & 0 & 0 & 0 & 0 & 0 & 0 & 0 & 18 & 3 & -11 & -18 & -18 & 0 & 0 & 0 \\
0 & 0 & 0 & 0 & 0 & 0 & 0 & 0 & 0 & 0 & 0 & 0 & 3 & 1 & 0 & 0 \\
0 & 0 & 0 & 0 & 0 & 0 & 0 & 0 & 0 & 0 & 0 & 0 & -3 & 0 & 1 & 0 \\
0 & 0 & 0 & 0 & 0 & 0 & 0 & 0 & 0 & 0 & 0 & 0 & 1 & 0 & 0 & 1 \\
1 & 0 & 0 & 0 & 0 & 0 & 0 & 0 & 0 & 0 & 0 & 0 & 0 & 0 & 0 & 0 \\
\alpha_{1} \\
\beta_{1} \\
\gamma_{1} \\
U_{2} \\
\alpha_{2} \\
\beta_{2} \\
\gamma_{2} \\
U_{3} \\
\alpha_{3} \\
\beta_{3} \\
\gamma_{3}
\end{array}\right)
$$

Cuja solução é

$$
\begin{gathered}
U_{0}=1, \quad \alpha_{0}=-\frac{405}{527}, \quad \beta_{0}=\frac{15}{527}, \quad \gamma_{0}=\frac{40}{527}, \\
U_{1}=\frac{177}{527}, \quad \alpha_{1}=-\frac{15}{31}, \quad \beta_{1}=\frac{135}{527}, \quad \gamma_{1}=-\frac{27}{527}, \\
U_{2}=\frac{30}{527}, \quad \alpha_{2}=-\frac{66}{527}, \quad \beta_{2}=\frac{54}{527}, \quad \gamma_{2}=-\frac{16}{527}, \\
U_{3}=\frac{2}{527}, \quad \alpha_{3}=-\frac{6}{527}, \quad \beta_{3}=\frac{6}{527}, \quad \gamma_{3}=-\frac{2}{527}
\end{gathered}
$$

E portanto a solução do problema é

$$
U(x, t)= \begin{cases}f_{0}\left(\frac{x}{\delta[t]}\right) & 0<\frac{x}{\delta[t]}<1 / 4 \\ f_{1}\left(\frac{x}{\delta[t]}\right) & 1 / 4<\frac{x}{\delta[t]}<1 / 2 \\ f_{2}\left(\frac{x}{\delta[t]}\right) & 1 / 2<\frac{x}{\delta[t]}<3 / 4 \\ f_{3}\left(\frac{x}{\delta[t]}\right) & 3 / 4<\frac{x}{\delta[t]}<1 \\ 0 & \text { c.c }\end{cases}
$$


para $\delta(t)=8 \sqrt{\frac{6}{13}} \sqrt{t}$ e $f_{i}$ dada por

$$
f_{i}(\xi)=U_{i}+\alpha_{i} \frac{\left(\xi-\xi_{i}\right)}{\left(\xi_{i+1}-\xi_{i}\right)}+\beta_{i} \frac{\left(\xi-\xi_{i}\right)^{2}}{\left(\xi_{i+1}-\xi_{i}\right)^{2}}+\gamma_{i} \frac{\left(\xi-\xi_{i}\right)^{3}}{\left(\xi_{i+1}-\xi_{i}\right)^{3}}, \quad i=0,1,2,3
$$

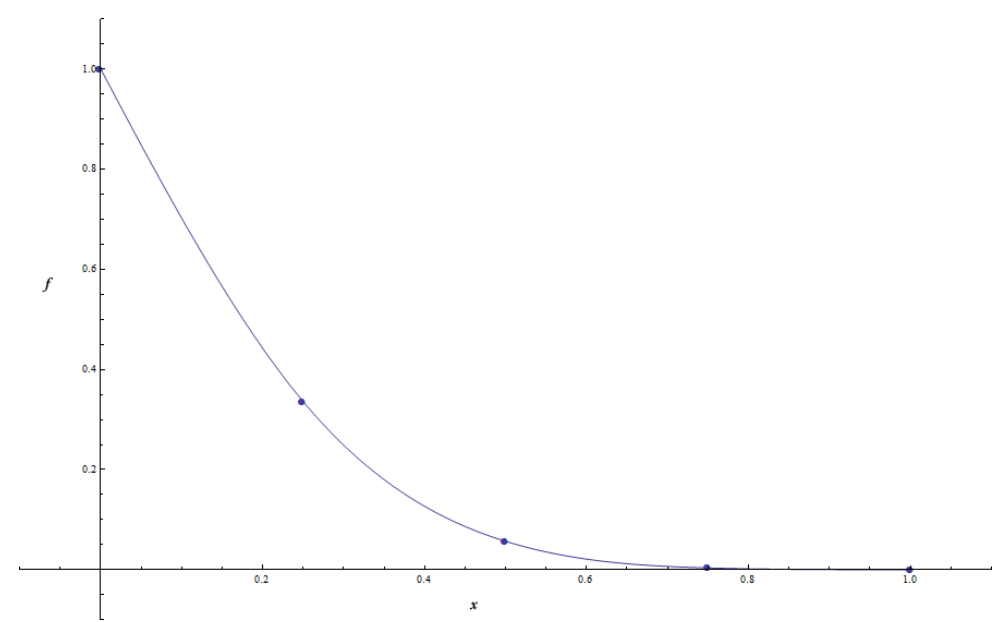

Figura 3.14: Gráfico das funções $f_{i}(\xi)$, com $\xi \in[0,1]$. Estão destacados os cinco pontos nodais $U_{i}$

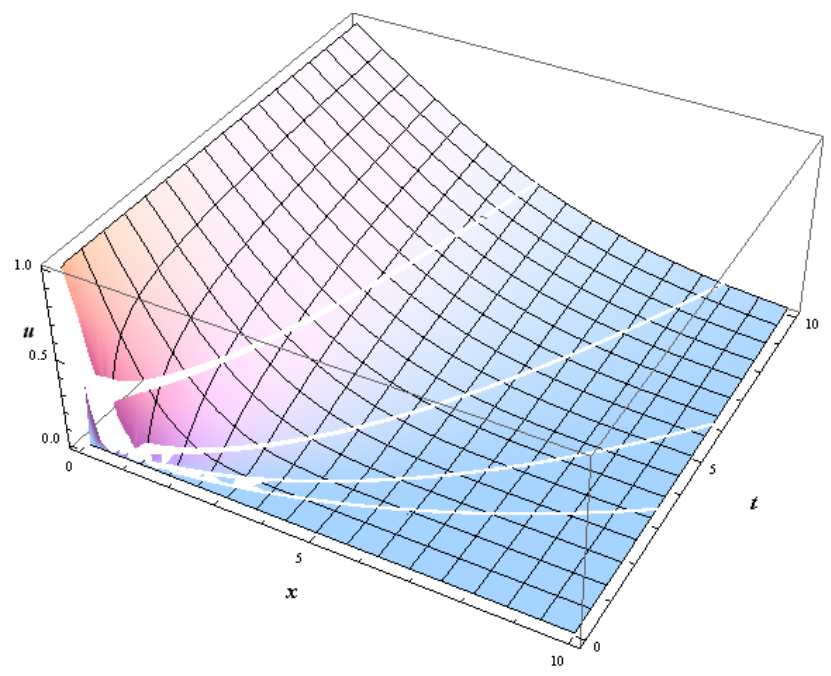

Figura 3.15: Gráfico da aproximação via HBIM nodal $(n=4)$ para a solução do problema 2.1 com temperatura constante na fronteira. Nesta imagem estão destacadas quatro curvas sobre os pontos nodais dos perfis cúbicos propostos para aproximação.

Em $t=1$, o erro máximo entre essa aproximação e a solução exata é menor que $3 \times 10^{-3}$.

\subsubsection{Fluxo constante}

A condição de fronteira de fluxo constante é muito similar à condição anterior. Após a imobilização de variáveis, com $\phi=\delta$, tem-se $U=\frac{1}{m}(1-\xi)^{m}$. Novamente, essa aproximação é independente do tempo, e portanto a spline que aproximará nos pontos nodais também será independente do tempo. Logo, $U_{t}=0$, e a equação diferencial é

$$
U_{\xi \xi}=\delta \delta_{t}\left(U-\xi U_{\xi}\right)
$$




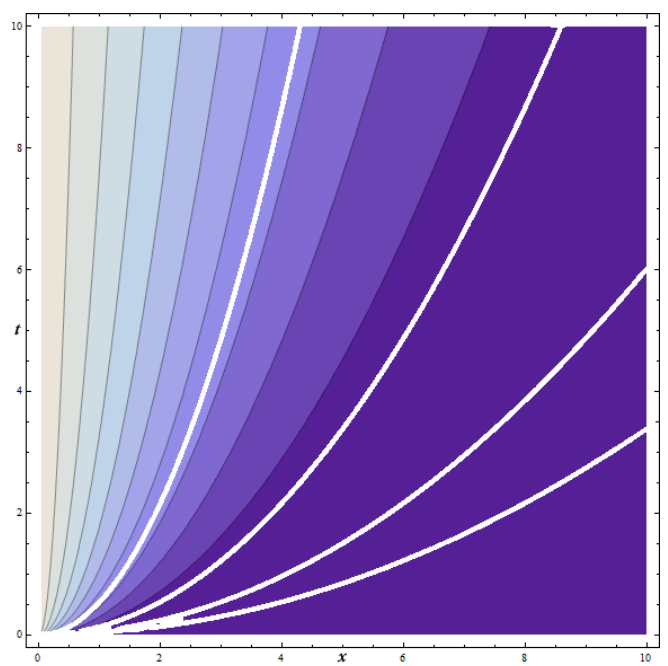

Figura 3.16: Curvas de nível da aproximação via HBIM nodal $(n=4)$ para a solução do problema 2.1 com temperatura constante na fronteira. Nesta imagem estão destacadas quatro curvas sobre os pontos nodais dos perfis cúbicos propostos para aproximação.

Em primeiro lugar, note que

$$
\int_{\xi_{i}}^{\xi_{i+1}} U-\xi \frac{\partial U}{\partial \xi} d \xi=\int_{\xi_{i}}^{\xi_{i+1}} U d \xi-\xi U+\int_{\xi_{i}}^{\xi_{i+1}} U d \xi=-\xi U+2 \int_{\xi_{i}}^{\xi_{i+1}} U d \xi
$$

Portanto a equação de balanceamento integral fica

$$
-\delta \delta_{t}\left(\xi_{i+1} U_{i+1}-\xi_{i} U_{i}-2 \int_{\xi_{i}}^{\xi_{i+1}} U d \xi\right)=\left.\frac{\partial U}{\partial \xi}\right|_{\xi=\xi_{i+1}}-\left.\frac{\partial U}{\partial \xi}\right|_{\xi=\xi_{i}}
$$

Substituindo o perfil cúbico, tem-se

$$
\begin{aligned}
-\delta \delta_{t}\left(\xi_{i+1} U_{i+1}-\xi_{i} U_{i}-2\left(\xi_{i+1}-\xi_{i}\right)\left(U_{i}+\frac{\alpha_{i}}{2}+\frac{\beta_{i}}{3}+\frac{\gamma_{i}}{4}\right)\right) & =n\left(2 \beta_{i}+3 \gamma_{i}\right) \\
-\delta \delta_{t}\left(\xi_{i+1} U_{i+1}+\left(\xi_{i}-2 \xi_{i+1}\right) U_{i}-\frac{2}{n}\left(\frac{\alpha_{i}}{2}+\frac{\beta_{i}}{3}+\frac{\gamma_{i}}{4}\right)\right) & =n\left(2 \beta_{i}+3 \gamma_{i}\right)-\delta \delta \\
t\left(\frac{i+1}{n} U_{i+1}-\frac{i+2}{n} U_{i}-\frac{2}{n}\left(\frac{\alpha_{i}}{2}+\frac{\beta_{i}}{3}+\frac{\gamma_{i}}{4}\right)\right) & =n\left(2 \beta_{i}+3 \gamma_{i}\right) \\
-\delta \delta_{t}\left((i+1) U_{i+1}-(i+2) U_{i}-2\left(\frac{\alpha_{i}}{2}+\frac{\beta_{i}}{3}+\frac{\gamma_{i}}{4}\right)\right) & =n^{2}\left(2 \beta_{i}+3 \gamma_{i}\right)
\end{aligned}
$$

E portanto,

$$
\delta \delta_{t}=\frac{n^{2}\left(2 \beta_{i}+3 \gamma_{i}\right)}{\alpha_{i}+\frac{2 \beta_{i}}{3}+\frac{\gamma_{i}}{2}-(i+1) U_{i+1}+(i+2) U_{i}}
$$

Fazendo $i=n-1$, tem-se:

$$
\delta \delta_{t}=\frac{2 n^{2}}{2 n-1}
$$

Igualando as duas últimas equações,

$$
3 \alpha_{i}+(3-2 n) \beta_{i}+3(1-n) \gamma_{i}-3(i+1) U_{i+1}+3(i+2) U_{i}=0
$$

Combinando a equação 3.20 com as equações 3.17 e 3.18, determinam-se as incógnitas. A con- 
dição de contorno $U_{\xi}=-1$ implica que $f_{0}^{\prime}\left(\xi_{0}\right)=-1$, ou seja, que $\alpha_{0}=-\frac{1}{n}$.

\subsubsection{Condição de Resfriamento}

Para a condição de resfriamento, tem-se o perfil aproximado $\frac{1}{m+\delta}(1-\xi)^{m}$ para a seguinte equação

$$
U_{\xi \xi}=\delta \delta_{t} U+\delta^{2} U_{t}-\xi \delta \delta_{t} U_{\xi}
$$

Esse perfil dependente do tempo sugere que as incógnitas $\alpha_{i}, \beta_{i}, \gamma_{i}$ e $U_{i}$ não sejam constantes no tempo. Integrando a equação sobre o intervalo $\left[\xi_{i}, \xi_{i+1}\right]$

$$
-\delta \delta_{t}\left(\xi_{i+1} U_{i+1}-\xi_{i} U_{i}-2 \int_{\xi_{i}}^{\xi_{i+1}} U d \xi\right)+\delta^{2} \frac{d}{d t} \int_{\xi_{i}}^{\xi_{i+1}} U d \xi=\left.\frac{\partial U}{\partial \xi}\right|_{\xi=\xi_{i+1}}-\left.\frac{\partial U}{\partial \xi}\right|_{\xi=\xi_{i}}
$$

e substituindo o perfil cúbico tem-se

$$
\delta \delta_{t}\left(\alpha_{i}+\frac{2 \beta_{i}}{3}+\frac{\gamma_{i}}{2}-(i+1) U_{i+1}+(i+2) U_{i}\right)+\delta^{2} \frac{d}{\mathrm{dt}}\left(\frac{\alpha_{i}}{2}+\frac{\beta_{i}}{3}+\frac{\gamma_{i}}{4}+U_{i}\right)=n^{2}\left(2 \beta_{i}+3 \gamma_{i}\right)
$$

A condição de fronteira $U_{\xi}=\delta U-1$ implica que $f_{0}^{\prime}\left(\xi_{0}\right)=\delta f_{0}\left(\xi_{0}\right)-1$, e portanto $n \alpha_{0}=\delta U_{0}-1$. Tem-se aqui um conjunto de $n$ EDOs, embora de fato sejam necessárias $4 n$ equações a fim de determinar todas as incógnitas. As outras EDOs são encontradas derivando as equações 3.17 e 3.18 com respeito a $t$. O sistema matricial é escrito $A x_{t}=b$, onde $x$ é definido como anteriormente, mas com uma entrada extra para $\delta$. Além disso, a condição de fronteira adicional para $\alpha_{0}$ é derivada com respeito a $t$ para dar a equação restante. No limite, à medida que $t \rightarrow 0$, as equações da condição de resfriamento se reduzem às de fluxo constante. Portanto, as condições iniciais para $x$ são justamente os valores encontrados para o caso de fluxo constante, junto de $\delta(0)=0$. As EDOs são resolvidas numericamente.

\subsubsection{Condição de Dirichlet}

Este método pode ser estendido para problemas com condição de Dirichlet arbitrária. Se impusermos $u(0, t)=h(t)$, a condição de fronteira da equação após a imobilização se torna $U=\frac{h}{\phi} \mathrm{e}$ portanto uma escolha natural de $\phi$ é $\phi=h(t)$, de modo que a nova condição seja $U(0, t)=1$, e a equação se torne

$$
h U_{\xi \xi}=\delta^{2} h_{t} U+\delta^{2} h U_{t}-\xi \delta \delta_{t} h U_{\xi}
$$

Depois de integrar a equação sobre $\left[\xi_{i}, \xi i+1\right]$, e substituir o perfil cúbico de aproximação, tem-se algo muito similar à condição de resfriamento.

$$
\begin{array}{r}
h \delta \delta_{t}\left(\frac{\alpha_{i}}{2}+\frac{\beta_{i}}{3}+\frac{\gamma_{i}}{4}-(i+1)\left(U_{i+1}-U_{i}\right)\right)+\delta^{2} h \frac{d}{\mathrm{dt}}\left(\frac{\alpha_{i}}{2}+\frac{\beta_{i}}{3}+\frac{\gamma_{i}}{4}+U_{i}\right) \\
=n^{2}\left(2 \beta_{i}+3 \gamma_{i}\right)-\delta^{2} h_{t}\left(\frac{\alpha_{i}}{2}+\frac{\beta_{i}}{3}+\frac{\gamma_{i}}{4}+U_{i}\right)
\end{array}
$$

Novamente, deve-se resolver o sistema $A x_{t}=b$, onde $A$ são as equações 3.21 junto de 3.17 e 3.18 , e a condição de fronteira $U_{1}=h$ (depois de diferenciar todas elas com respeito a $t$ ). Como $h(0)=1$, as equações para essa condição de fronteira se reduzem àquelas para a condição de fronteira de temperatura constante, no limite $t \rightarrow 0$. Portanto, estes coeficientes serão as condições iniciais. 


\section{Capítulo 4}

\section{O Problema de Stefan}

Atualmente, uma ampla classe de problemas contendo fronteiras móveis ou fronteiras livres é conhecida por problemas de Stefan (referente a Jozef Stefan, 1835-1893). O seguinte resumo histórico é baseado nas ideias do trabalho Crepeau (2007) que possui uma concisa análise da modelagem e do estudo desses problemas.

\subsection{Apresentação do problema e o Problema unidimensional de uma fase.}

Stefan iniciou sua pesquisa investigando a formação de gelo nos mares polares. Em vez de calcular a transferência de calor através de uma fronteira fixa, a modelagem da taxa de crescimento de gelo carecia uma dependência no tempo ou uma fronteira móvel. Ao aplicar um modelo simples de conservação de energia na interface sólido-líquido, Stefan pôde perceber que, à medida que o líquido se tornava sólido, uma quantidade de calor por unidade de área era liberada, $L_{m} \rho d s$. E ao assumir um perfil linear de temperatura no gelo, aplicou a lei de Fourier para calcular a quantidade de calor conduzido para fora da frente de congelamento por unidade de área, $k \frac{\Delta u}{s(t)} d t$, onde $\Delta u$ era a diferença de temperatura entre a interface água-gelo e a a interface gelo-ar. Em uma primeira análise, a temperatura da interface gelo-ar permanecia constante. A experiência de Stefan para medir a condutividade térmica usando métodos que dependiam do tempo serviram bem para seu modelo. Equacionando a liberação do calor latente com a quantidade conduzida para fora da frente de congelamento, Stefan mostrou que o quadrado da espessura do gelo era uma função linear do tempo,

$$
s^{2}(t)=\frac{2}{\rho} \frac{k \Delta u}{L_{m}} t
$$

Sabendo que essa era apenas uma simplificação do modelo físico, Stefan modelou a temperatura do ar acima da interface gelo-ar como uma função do tempo e assumiu a ela a função de temperatura de interface. A solução desse problema foi

$$
s^{2}(t)=\frac{2 k}{\rho L_{m}} \int_{0}^{t} \Delta u d t
$$

Este modelo concordava melhor com os dados coletados disponíveis. Surgia, então, o modelo matemático para o problema de fronteira móvel, hoje conhecido por problema de Stefan. Dada a 
equação de difusão

$$
\frac{\partial u(x, t)}{\partial u}=\frac{k}{\rho c_{p}} \frac{\partial^{2} u(x, t)}{\partial x^{2}}
$$

Stefan definiu o local da interface gelo-ar em $x=0$ e o local da interface gelo-líquido em $x=s(t)$. A temperatura na interface gelo-líquido era zero e a a temperatura na interface gelo-ar era $u=f(t)$, sendo $f$ uma distribuição de temperatura qualquer. O balanço do calor na interface gelo-líquido era

$$
\rho L_{m} \frac{d s}{d t}=-\left.k \frac{\partial u}{\partial x}\right|_{x=s}
$$

Expressando a temperatura como um diferencial total

$$
d T=\frac{\partial u}{\partial t}+\left.\frac{\partial u}{\partial x}\right|_{x=s} \frac{d s}{d t}=0
$$

é possível eliminar o termo $\frac{d s}{d t}$ e obter

$$
\frac{\partial u}{\partial t}=\frac{k}{\rho L_{m}}\left(\left.\frac{\partial u}{\partial x}\right|_{x=s}\right)^{2}
$$

cuja solução com constantes de integração $C$ e $\alpha$ é

$$
u(x, t)=C \int_{\frac{x}{2 \sqrt{\frac{k}{\rho c p}} t}}^{\alpha} e^{-z^{2}} d z
$$

Aplicando a condição de contorno $u(x=0, t)=f(t)=\Delta u$, Stefan obteve

$$
\Delta u=C \int_{0}^{\alpha} e^{-z^{2}} d z
$$

E na interface gelo-líquido, $u(x=s(t), t)=0$, obteve

$$
s(t)=2 \alpha \sqrt{\frac{k}{\rho c_{p}} t}
$$

Para eliminar a constante $C$, Stefan calculou a derivada temporal e a espacial da equação 4.2

$$
\begin{aligned}
& \frac{\partial u}{\partial t}=\frac{C x}{4 t \sqrt{\frac{k}{\rho c_{p}} t} \exp \left[-\frac{x^{2}}{4\left(k / \rho c_{p}\right) t}\right]} \\
& \frac{\partial u}{\partial x}=\frac{-C}{2 \sqrt{\frac{k}{\rho c_{p}} t}} \exp \left[-\frac{x^{2}}{4\left(k / \rho c_{p}\right) t}\right]
\end{aligned}
$$

Substituindo de volta na equação 4.1, obteve

$$
\frac{C x}{4 t \sqrt{\frac{k}{\rho c_{p}} t}} \exp \left[-\frac{x^{2}}{4\left(k / \rho c_{p}\right) t}\right]==\frac{k}{\rho L_{m}}\left(\frac{-C}{2 \sqrt{\frac{k}{\rho c_{p}} t}} \exp \left[-\frac{x^{2}}{4\left(k / \rho c_{p}\right) t}\right]\right)^{2}
$$

E portanto, 


$$
\frac{C e^{-\frac{x^{2}}{4\left(k / \rho c_{p}\right) t}} x}{4 t \sqrt{\frac{k t}{\rho c_{p}}}}=\frac{C^{2} e^{-\frac{x^{2}}{2\left(k / \rho c_{p}\right) t}} c_{p}}{4 t L_{m}}
$$

Isolando $C$ e utilizando a equação 4.3

$$
\Delta u=\int_{0}^{\alpha} e^{-z^{2}} \frac{e^{\frac{x^{2}}{4(k / \rho c p) t}} x L_{m}}{\sqrt{\frac{k t}{\rho c_{p}}} c_{p}} d z
$$

Como a análise se dá em $x=s(t)=2 \alpha \sqrt{\frac{k}{\rho c_{p}} t}, \frac{x}{2 \alpha}=\sqrt{\frac{k}{\rho c_{p}} t}$ e portanto

$$
\Delta u=\int_{0}^{\alpha} e^{-z^{2}} \frac{e^{\frac{x^{2}}{x^{2} / \alpha^{2}}} x L_{m}}{\frac{x}{2 \alpha} c_{p}} d z
$$

ou ainda,

$$
1=\int_{0}^{\alpha} e^{-z^{2}} \alpha e^{\alpha^{2}} \frac{2 L_{m}}{\Delta u c_{p}} d z
$$

resultando na equação transcendental em $\alpha$

$$
\sqrt{\pi} \beta \alpha e^{\alpha^{2}} \operatorname{erf}(\alpha)=1
$$

onde $\beta$ é o número de Stefan $\beta=\frac{L_{m}}{c_{p} \Delta u}$.

A mesma modelagem se adequa ao problema de derretimento de uma lâmina de gelo semiinfinita. Inicialmente à temperatura de derretimento (indicada por zero), a temperatura da superfície é aumentada em tempo $t=0$ a uma temperatura acima de zero, subsequentemente mantida.

Uma simplificação para esse problema é uma adimensionalização em que o único parâmetro restante é exatamente o número de Stefan. Com a seguinte condição de fronteira, um dos problemas de Stefan mais simples é o caso do material semi-infinito unidimensional em temperatura de derretimento:

$$
\begin{gathered}
\frac{\partial u}{\partial t}=\frac{\partial^{2} u}{\partial x^{2}}, 0<x<s(t) \\
u(0, t)=1, u(s, t)=0, \beta \frac{d s}{d t}=-\left.\frac{\partial u}{\partial x}\right|_{x=s}, s(0)=0 ;
\end{gathered}
$$

Este é um dos poucos problemas dessa classe que possui uma solução exata, a saber

$$
u(x, t)=1-\frac{\operatorname{erf}\left(\frac{x}{2 \sqrt{t}}\right)}{\operatorname{erf}(\alpha)}, \quad 0 \leq x \leq s(t), \quad s(t)=2 \alpha \sqrt{t}
$$

em que $\alpha$ satisfaz a equação transcendental 4.4.

A seguir, a figura 4.1 mostra um gráfico da solução do problema com $\beta=1$ e a figura 4.2 mostra as curvas de nível da solução. Nesse caso, $\alpha$ é aproximadamente 0.62. É possível notar nesses gráficos claramente a curva $s(t)=2 \alpha \sqrt{t}$. 


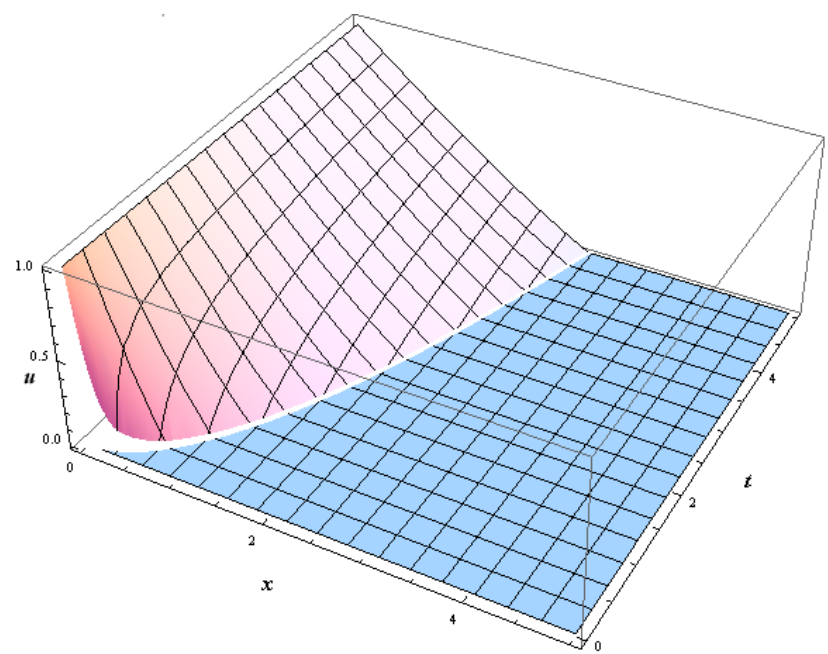

Figura 4.1: Solução do Problema de Stefan com temperatura constante na fronteira e $\beta=1$. É possível notar a lacuna da fronteira móvel, inerente ao problema.

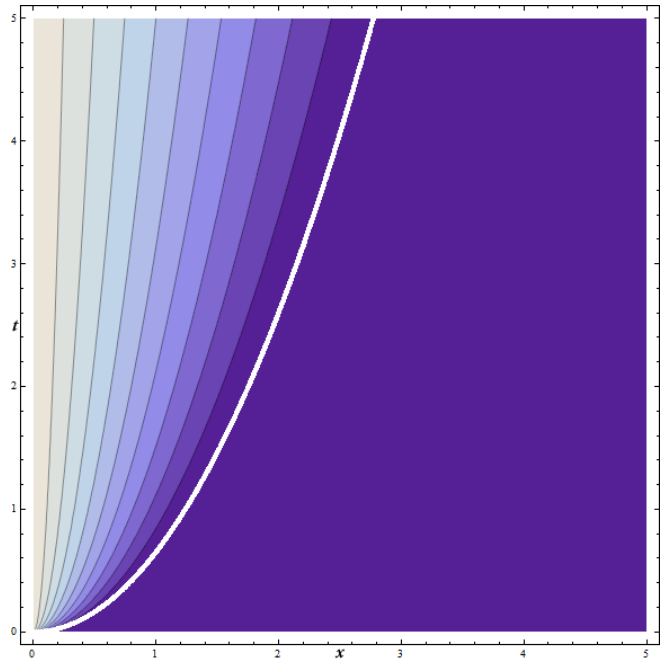

Figura 4.2: Curvas de nível da solução do Problema de Stefan com temperatura constante na fronteira e $\beta=1$

\subsection{Soluções Aproximadas}

Os métodos apresentados nos capítulos anteriores serão neste capítulo aplicados para os problemas de Stefan. As aplicações são muitas vezes imediatas, pois pode-se fazer uma correspondência direta entre a profundidade de penetração $\delta$ dos métodos estudados com a fronteira móvel dos problemas de Stefan. A diferença está no fato de a fronteira móvel também ser uma incógnita do problema. Entretanto, a condição de Stefan proporciona mais uma equação a fim de determinar a posição da fronteira móvel.

\subsubsection{O método de Balanceamento Integral}

Um perfil de aproximação típico para o problema de Stefan é

$$
u=a\left(1-\frac{x}{s}\right)+(1-a)\left(1-\frac{x}{s}\right)^{m}
$$


onde $s$ é a fronteira móvel. Nesse caso, a condição de fronteira em 0 é $u(0, t)=1$ e na fronteira móvel $u(s, t)=0$. Esse perfil difere dos perfis apresentados nos problemas termais pelo fato de não ser um monômio e apresentar um termo linear. Esse termo é necessário pois, caso contrário, tem-se $u_{x}(s, t)=0$ para $m>1$ e a frente ficaria estacionária para todo tempo. Para alguns problemas, os valores de $a$ e de $m$ podem variar com o tempo, mas para uma análise simplificada, eles serão sempre assumidos constantes. Apenas caso apareçam inconsistências devido a essa suposição eles serão dependentes do tempo.

Novamente, o problema de Stefan

$$
\begin{aligned}
\frac{\partial^{2} u}{\partial x^{2}} & =\frac{\partial u}{\partial t}, \quad 0<x<s(t) \\
\beta \frac{d s}{d t} & =-\frac{\partial u}{\partial x}, \quad \text { em } x=s \\
u & =0, \quad \text { em } x=s \\
u(x, 0) & =0, \quad \text { para } x>0
\end{aligned}
$$

com condições de fronteira

$$
u=1 \quad \text { ou } \quad \frac{\partial u}{\partial x}=-\gamma_{1}+\gamma_{2}(u-1) \quad \text { em } x=0
$$

Aqui, a segunda condição de fronteira é uma condição de Robin genérica que incorpora tanto a condição de fluxo constante $\left(\gamma_{1}=1\right.$ e $\left.\gamma_{2}=0\right)$ quanto a condição de resfriamento $\left(\gamma_{1}=0\right.$ e $\left.\gamma_{2}=1\right)$. Para a condição de temperatura constante existe uma solução exata. Para a condição de Robin não há solução analítica conhecida.

O método de balanceamento integral envolve integrar a equação 4.8 com respeito a $x$ sobre o intervalo $(0, s(t))$. Isso dá:

$$
\left.\frac{\partial u}{\partial x}\right|_{x=s}-\left.\frac{\partial u}{\partial x}\right|_{x=s}=\frac{d}{d t} \int_{0}^{s} u d x
$$

uma vez que $u=0$ em $x=s$ ao calcular a derivada do termo da integral. Uma vez definido o perfil de aproximação, a equação integral deve ser resolvida em conjunto com a condição de Stefan. Uma condição de Stefan alternativa sugerida por Goodman (1958) vem de $u(s(t), t)=0$ para todo $t$, e portanto a derivada total em $x=s(t)$ é zero, isto é:

$$
\frac{D}{D t} u(s(t), t)=0 \Rightarrow \frac{\partial u}{\partial t}+\frac{\partial u}{\partial x} \frac{d s}{d t}=0
$$

ou seja

$$
\begin{aligned}
\frac{\partial u}{\partial t}+\frac{\partial u}{\partial x} \frac{\mathrm{ds}}{\mathrm{dt}} & =0 \\
\frac{\partial^{2} u}{\partial x^{2}}+\frac{\partial u}{\partial x}\left(-\frac{1}{\beta} \frac{\partial u}{\partial x}\right) & =0 \\
\beta \frac{\partial^{2} u}{\partial x^{2}} & =\left(\frac{\partial u}{\partial x}\right)^{2}, \quad \text { em } x=s
\end{aligned}
$$




\section{Temperatura constante na fronteira}

Como notado antes, a solução para o problema com condição de fronteira $u(0, t)=1$ é

$$
u(x, t)=1-\frac{\operatorname{erf}\left(\frac{x}{2 \sqrt{t}}\right)}{\operatorname{erf}(\alpha)}, \quad s(t)=2 \alpha \sqrt{t},
$$

onde $\alpha$ satisfaz a equação transcendental

$$
\sqrt{\pi} \beta \alpha e^{\alpha^{2}} \operatorname{erf}(\alpha)=1
$$

A função originalmente proposta por Goodman (1958) era um polinômio quadrático da forma $u=a(x-s)+b(x-s)^{2}$. Contudo, Wood (2001) sugere a formulação

$$
u(x, t)=a\left(1-\frac{x}{s}\right)+b\left(1-\frac{x}{s}\right)^{2}
$$

pois esse garante coeficientes constantes no tempo. De fato, isso apenas ocorre para este tipo de condição de fronteira: para a condição de Robin, eles dependem do tempo. Ambas formulações satisfazem automaticamente $u(s, t)=0$. Aplicando $u(0, t)=1$ dá $b=1-a$ e portanto

$$
u(x, t)=a\left(1-\frac{x}{s}\right)+(1-a)\left(1-\frac{x}{s}\right)^{2}
$$

Para o perfil aproximado tem-se

$$
\frac{\partial u}{\partial x}=-\frac{a}{s}-\frac{1-a}{s}\left(1-\frac{x}{s}\right)
$$

$\mathrm{e}$

$$
\int u d x=-\frac{a s}{2}\left(1-\frac{x}{s}\right)^{2}-\frac{s(1-a)}{3}\left(1-\frac{x}{s}\right)^{3}
$$

e, portanto,

$$
\left.\frac{\partial u}{\partial x}\right|_{x=s}=-\frac{a}{s},\left.\quad \frac{\partial u}{\partial x}\right|_{x=0}=\frac{a-2}{s}, \quad \int_{0}^{s} u d x=\frac{s(a+2)}{6}
$$

enquanto a condição de Stefan fica

$$
s \frac{d t}{d s}=\frac{a}{\beta}
$$

A equação integral, então, é (assumindo $a$ constante)

$$
\begin{aligned}
-\frac{a-2}{s}-\frac{a}{s} & =\frac{(a+2)}{6} \frac{d s}{d t} \\
-12 \frac{a-1}{a+2} & =s \frac{d s}{d t}
\end{aligned}
$$

que unida com a condição de Stefan, resulta em

$$
-12 \frac{a-1}{a+2}=\frac{a}{\beta}
$$

ou ainda

$$
a=-1-6 \beta+\sqrt{1+24 \beta+36 \beta^{2}}
$$


onde a raiz positiva foi escolhida para garantir que $u_{x}(s, t)<0$. Como $a$ é suposto constante, é possível concluir que, como na solução exata, $s \sim t^{1 / 2}$,

$$
s(t)=2 \alpha \sqrt{t}, \quad \alpha=\sqrt{\frac{a}{2 \beta}}
$$

e portanto a solução aproximada pelo método HBIM é

$$
u(x, t)=a\left(1-\frac{x}{2 \alpha \sqrt{t}}\right)+(1-a)\left(1-\frac{x}{2 \alpha \sqrt{t}}\right)^{2}
$$

Goodman (1958) propõe duas diferentes formulações e Wood (2001) apresenta seis formulações distintas para o método HBIM. Cada formulação envolve diferentes escolhas para a condição de Stefan e diversas opções de aproximação da derivada $u_{x}(s, t)$ na equação integral. A eficácia destas variações depende fortemente do número de Stefan $\beta$ do problema. Mitchell e Myers (2010a) aponta que para o caso do gelo, em que $\Delta u<100 K$, o método descrito é normalmente o mais preciso.

Numa tentativa de melhorar a precisão do método, Myers et al. (2007) emprega o uso de um perfil cúbico. De uma forma geral, o perfil cúbico pode ser apresentado como

$$
u(x, t)=a\left(1-\frac{x}{s}\right)+b\left(1-\frac{x}{s}\right)^{2}+c\left(1-\frac{x}{s}\right)^{3}
$$

em que $a, b$ e $c$ são constantes para o caso de temperatura constante na fronteira. A expressão satisfaz automaticamente $u(s, t)=0$ e para que $u(0, t)=1$, é necessário $c=1-a-b$. Agora há mais um coeficiente que no caso quadrático, e se faz uso da forma alternativa da condição de Stefan para encontrar $b=\frac{a^{2}}{2 \beta}$. O perfil aproximado se torna

$$
u(x, t)=a\left(1-\frac{x}{s}\right)+\frac{a^{2}}{2 \beta}\left(1-\frac{x}{s}\right)^{2}+\left(1-a-\frac{a^{2}}{2 \beta}\right)\left(1-\frac{x}{s}\right)^{3}
$$

Novamente, o problema reduz-se a encontrar o valor para $a$ e a frente móvel $s(t)$. Para o perfil cúbico tem-se

$$
\frac{\partial u}{\partial x}=-\frac{a}{s}-\frac{a^{2}}{s \beta}\left(1-\frac{x}{s}\right)-\frac{3}{s}\left(1-a-\frac{a^{2}}{2 \beta}\right)\left(1-\frac{x}{s}\right)^{2}
$$

e

$$
\int u d x=-\frac{a s}{2}\left(1-\frac{x}{s}\right)^{2}-\frac{s a^{2}}{6 \beta}\left(1-\frac{x}{s}\right)^{3}-\frac{s}{4}\left(1-a-\frac{a^{2}}{2 \beta}\right)\left(1-\frac{x}{s}\right)^{4}
$$

$\log 0$

$$
\left.\frac{\partial u}{\partial x}\right|_{x=s}=-\frac{a}{s},\left.\quad \frac{\partial u}{\partial x}\right|_{x=0}=-\frac{a}{s}-\frac{a^{2}}{s \beta}-\frac{3}{s}\left(1-a-\frac{a^{2}}{2 \beta}\right)
$$

e

$$
\int_{0}^{s} u d x=-\frac{a s}{2}-\frac{s a^{2}}{6 \beta}-\frac{s}{4}\left(1-a-\frac{a^{2}}{2 \beta}\right)
$$

Substituindo esses valores na equação integral, tem-se

$$
\begin{aligned}
-\frac{a}{s}-\left(-\frac{a}{s}-\frac{a^{2}}{s \beta}-\frac{3}{s}\left(1-a-\frac{a^{2}}{2 \beta}\right)\right) & =-\frac{a s}{2}-\frac{s a^{2}}{6 \beta}-\frac{s}{4}\left(1-a-\frac{a^{2}}{2 \beta}\right) \\
\frac{a^{2}}{s \beta}+\frac{3}{s}\left(1-a-\frac{a^{2}}{2 \beta}\right) & =\frac{d}{d t}\left(-\frac{a s}{2}-\frac{s a^{2}}{6 \beta}-\frac{s}{4}\left(1-a-\frac{a^{2}}{2 \beta}\right)\right)
\end{aligned}
$$




$$
\begin{aligned}
-\frac{a^{2}}{\beta}-3\left(1-a-\frac{a^{2}}{2 \beta}\right) & =\left(-\frac{a}{2}-\frac{a^{2}}{6 \beta}-\frac{1}{4}\left(1-a-\frac{a^{2}}{2 \beta}\right)\right) s \frac{d s}{d t} \\
\frac{\frac{a^{2}-6 \beta+6 a \beta}{2 \beta}}{-\frac{a^{2}+6(1+a) \beta}{24 \beta}} & =s \frac{d s}{d t}
\end{aligned}
$$

E obtém-se a equação diferencial para $s$

$$
s \frac{d s}{d t}=-12 \frac{a^{2}-6 \beta+6 a \beta}{a^{2}+6 a \beta+6 \beta}
$$

Para encontrar o valor de $a$ (suposto constante), basta utilizar a condição de Stefan, de modo que

$$
\frac{a}{\beta}=-12 \frac{a^{2}-6 \beta+6 a \beta}{a^{2}+6 a \beta+6 \beta}
$$

o que implica que

$$
a^{3}+6 a^{2} \beta+6 a \beta=12 a^{2} \beta+72 \beta^{2}-72 a \beta^{2}
$$

ou ainda

$$
a^{3}+18 \beta a^{2}+6 \beta(1+12 \beta) a-72 \beta^{2}=0
$$

Uma vez determinado $a$ a partir dessa última equação, tem-se a solução para $s$, novamente $s(t)=$ $2 \alpha \sqrt{t}$, mas agora com outro valor para $a$.

Outro perfil de aproximação (que até o momento não foi citado para métodos de aproximação de solução de problemas termais) é o perfil exponencial. Mosally et al. (2002) buscam a motivação de tal perfil na solução exata do problema, notando que a expansão da função erro é erf $z \sim \frac{2}{\sqrt{\pi}} z e^{-z^{2}}$. Consequentemente, é escolhido para aproximação

$$
u=a+\frac{b x}{s} e^{-c \frac{x^{2}}{s^{2}}}
$$

Impondo $u(s, t)=0$ e $u(0, t)=1$, tem-se

$$
u=1-\frac{x}{s} e^{c\left(1-x^{2} / s^{2}\right)}
$$

Para este perfil:

$$
\frac{\partial u}{\partial x}=\left(\frac{2 c x^{2}-s^{2}}{s^{3}}\right) e^{c\left(1-x^{2} / s^{2}\right)}
$$

$\mathrm{e}$

$$
\int u d x=\frac{s}{2 c} e^{c\left(1-x^{2} / s^{2}\right)}+x
$$

Assim, na equação integral do método HBIM, tem-se

$$
\frac{2 c-1}{s}-\frac{e^{c}}{s}=\frac{d}{d t}\left(s-\frac{\left(e^{c}-1\right) s}{2 c}\right)
$$

ou seja

$$
s \frac{d s}{d t}\left(1-\frac{e^{c}-1}{2 c}\right)=2 c-1-e^{c}
$$


Pela condição de Stefan,

$$
s \frac{d s}{d t}=\frac{1-2 c}{\beta}
$$

então substituindo na equação anterior, tem-se

$$
\begin{aligned}
\frac{1-2 c}{\beta}\left(1-\frac{e^{c}-1}{2 c}\right) & =2 c-1-e^{c} \\
\frac{1-2 c}{\beta}\left(1-\frac{e^{c}-1}{2 c}\right)+1-2 c & =-e^{c} \\
(1-2 c)\left(\frac{1}{\beta}\left(1-\frac{e^{c}-1}{2 c}\right)+1\right) & =-e^{c} \\
(1-2 c)\left(\left(1-\frac{e^{c}-1}{2 c}\right)+\beta\right) & =-\beta \mathrm{e}^{c} \\
(1-2 c)\left(2 c-e^{c}+1+2 c \beta\right) & =-2 \mathrm{c} \beta \mathrm{e}^{c} \\
(1-2 c)\left(2 c(1+\beta)-e^{c}+1\right) & =-2 \mathrm{c} \beta \mathrm{e}^{c}
\end{aligned}
$$

$\log$,

$$
(2 c-1)\left(2 c(1+\beta) e^{-c}-1+e^{-c}\right)=2 c \beta
$$

A solução para $s$ aqui é $s(t)=2 \sqrt{\frac{1-2 c}{2 \beta}} \sqrt{t}$, com o valor de $c$ dado pela equação anterior.

\section{Condição de fronteira de Robin}

Novamente serão considerados os perfis quadrático, cúbico e exponencial mas agora para uma condição de fronteira de Robin. Primeiro note que para o perfil quadrático 4.14 tem-se

$$
\begin{gathered}
u=a\left(1-\frac{x}{s}\right)+b\left(1-\frac{x}{s}\right)^{2} \\
u_{x}=-\frac{a}{s}-\frac{2 b}{s}\left(1-\frac{x}{s}\right)
\end{gathered}
$$

E portanto em $x=0$

$$
\begin{gathered}
u(0, t)=a+b \\
u_{x}(0, t)=-\frac{a}{s}-\frac{2 b}{s}
\end{gathered}
$$

Ou seja, substituindo na condição de fronteira de Robin 4.12

$$
-\frac{a}{s}-\frac{2 b}{s}=-\gamma_{1}+\gamma_{2}(a+b-1)
$$

Escreve-se $b$ em função de $a$ e $\gamma$

$$
b=\frac{s\left(\gamma_{1}+\gamma_{2}\right)-a\left(1+s \gamma_{2}\right)}{2+s \gamma_{2}}
$$

E a solução aproximada fica

$$
u=a\left(1-\frac{x}{s}\right)+\frac{s\left(\gamma_{1}+\gamma_{2}\right)-a\left(1+s \gamma_{2}\right)}{2+s \gamma_{2}}\left(1-\frac{x}{s}\right)^{2}
$$


Para esta aproximação tem-se

$$
\frac{\partial u}{\partial x}=-\frac{a}{s}-2 \frac{s\left(\gamma_{1}+\gamma_{2}\right)-a\left(1+s \gamma_{2}\right)}{s\left(2+s \gamma_{2}\right)}\left(1-\frac{x}{s}\right)
$$

e também

$$
\int u d x=-\frac{s a}{2}\left(1-\frac{x}{s}\right)^{2}-\frac{s}{3} \frac{s\left(\gamma_{1}+\gamma_{2}\right)-a\left(1+s \gamma_{2}\right)}{2+s \gamma_{2}}\left(1-\frac{x}{s}\right)^{3}
$$

Substituindo na equação integral do HBIM, tem-se

$$
-\frac{a}{s}-\left(-2 \frac{\left(\gamma_{1}+\gamma_{2}\right) s-a\left(\gamma_{2} s+1\right)}{s\left(\gamma_{2} s+2\right)}-\frac{a}{s}\right)=\frac{d}{d t}\left(\frac{s\left(2\left(\gamma_{1}+\gamma+2\right) s+\left(\gamma_{2} s+4\right) a\right)}{6\left(\gamma_{2} s+2\right)}\right)
$$

ou ainda

$$
2 \frac{\left(\gamma_{1}+\gamma_{2}\right) s-a\left(\gamma_{2} s+1\right)}{s\left(\gamma_{2} s+2\right)}=\frac{d}{d t}\left(\frac{s\left(2\left(\gamma_{1}+\gamma+2\right) s+\left(\gamma_{2} s+4\right) a\right)}{6\left(\gamma_{2} s+2\right)}\right)
$$

Essa equação deve ser resolvida em conjunto com a condição de Stefan. Não é possível assumir $a$ constante nesse caso, e portanto há duas equações diferenciais a resolver para as variáveis $a(t)$ e $s(t)$. Como $s(0)=0$, é claro que deve-se ter $a(0)=0$, de modo que a condição inicial $u(x, 0)=0$ seja satisfeita.

Para o perfil cúbico tem-se

$$
\begin{gathered}
u(x, t)=a\left(1-\frac{x}{s}\right)+b\left(1-\frac{x}{s}\right)^{2}+c\left(1-\frac{x}{s}\right)^{3} \\
u_{x}=-\frac{a}{s}-\frac{2 b}{s}\left(1-\frac{x}{s}\right)-\frac{3 c}{s}\left(1-\frac{x}{s}\right)^{2} \\
u_{x x}=\frac{2 b}{s^{2}}+\frac{6 c}{s^{2}}\left(1-\frac{x}{s}\right)
\end{gathered}
$$

E portanto em $x=0$

$$
\begin{gathered}
u(0, t)=a+b+c \\
u_{x}(0, t)=-\frac{a}{s}-\frac{2 b}{s}-\frac{3 c}{s}
\end{gathered}
$$

E em $x=s$

$$
\begin{gathered}
u(s, t)=0 \\
u_{x}(s, t)=-\frac{a}{s} \\
u_{x x}(s, t)=\frac{2 b}{s^{2}}
\end{gathered}
$$

Usando a condição de Stefan alternativa $(\mathrm{em} x=s)$

$$
\begin{aligned}
u_{x}^{2} & =\beta u_{x x} \\
\left(-\frac{a}{s}\right)^{2} & =\beta \frac{2 b}{s^{2}} \\
\frac{a^{2}}{2 \beta} & =b
\end{aligned}
$$


Substituindo na condição de fronteira de Robin 4.12

$$
-\frac{a}{s}-\frac{a^{2}}{\beta s}-\frac{3 c}{s}=-\gamma_{1}+\gamma_{2}\left(a+\frac{a^{2}}{2 \beta}+c-1\right)
$$

Escrevendo $c$ em função de $a$ e $\gamma$

$$
c=\frac{\left(\gamma_{1}+\gamma_{2}\right) s-\left(1+\gamma_{2} s\right) a-\frac{a^{2}}{2 \beta}\left(2+\gamma_{2} s\right)}{3+s \gamma_{2}}
$$

E a solução aproximada fica

$$
u(x, t)=a\left(1-\frac{x}{s}\right)+\frac{a^{2}}{2 \beta}\left(1-\frac{x}{s}\right)^{2}+\frac{\left(\gamma_{1}+\gamma_{2}\right) s-\left(1+\gamma_{2} s\right) a-\frac{a^{2}}{2 \beta}\left(2+\gamma_{2} s\right)}{3+s \gamma_{2}}\left(1-\frac{x}{s}\right)^{3}
$$

Para esta aproximação tem-se

$$
\frac{\partial u}{\partial x}=-\frac{a}{s}-\frac{a^{2}}{\beta s}\left(1-\frac{x}{s}\right)-\frac{3}{s} \frac{\left(\gamma_{1}+\gamma_{2}\right) s-\left(1+\gamma_{2} s\right) a-\frac{a^{2}}{2 \beta}\left(2+\gamma_{2} s\right)}{3+s \gamma_{2}}\left(1-\frac{x}{s}\right)^{2}
$$

e também

$$
\int u d x=-\frac{a s}{2}\left(1-\frac{x}{s}\right)^{2}-\frac{s a^{2}}{6 \beta}\left(1-\frac{x}{s}\right)^{3}-\frac{s}{4} \frac{\left(\gamma_{1}+\gamma_{2}\right) s-\left(1+\gamma_{2} s\right) a-\frac{a^{2}}{2 \beta}\left(2+\gamma_{2} s\right)}{3+s \gamma_{2}}\left(1-\frac{x}{s}\right)^{4}
$$

Substituindo na equação integral do HBIM, tem-se

$$
\begin{array}{r}
-\frac{a}{s}-\left(-\frac{a}{s}-\frac{a^{2}}{\beta s}-\frac{3}{s} \frac{\left(\gamma_{1}+\gamma_{2}\right) s-\left(1+\gamma_{2} s\right) a-\frac{a^{2}}{2 \beta}\left(2+\gamma_{2} s\right)}{3+s \gamma_{2}}\right)= \\
\frac{d}{d t}\left(s \frac{6 \beta\left(\gamma_{1}+\gamma_{2}\right) s+6 \beta\left(5+\gamma_{2} s\right) a+a^{2}\left(6+\gamma_{2} s\right)}{24 \beta\left(3+\gamma_{2} s\right)}\right)
\end{array}
$$

ou ainda

$$
\left.\frac{6 \beta\left(\gamma_{1}+\gamma_{2}\right) s-6 \beta\left(1+\gamma_{2} s\right) a-a^{2} \gamma_{2} s}{2 \beta s\left(3+\gamma_{2} s\right)}=\frac{d}{d t}\left(s \frac{6 \beta\left(\gamma_{1}+\gamma_{2}\right) s+6 \beta\left(5+\gamma_{2} s\right) a+a^{2}\left(6+\gamma_{2} s\right)}{24 \beta\left(3+\gamma_{2} s\right)}\right)\right)
$$

Unida à condição de Stefan, obtém-se um par de equações diferenciais para $a(t)$ e $s(t)$. Novamente, a condição para $a$ é $a(0)=0$.

Por fim, para o perfil exponencial

$$
u=1-\frac{x}{s} e^{c\left(1-x^{2} / s^{2}\right)}
$$

tem-se, aplicando a condição de fronteira de Robin,

$$
u=\frac{\left(\gamma_{1}+\gamma_{2}\right) s e^{-c}}{\gamma_{2} s e^{-c}+1}\left(1-\frac{x}{s} e^{c\left(1-x^{2} / s^{2}\right)}\right)
$$

e, substituindo na equação integral, resulta em

$$
\frac{\left(\gamma_{1}+\gamma_{2}\right)\left(1-e^{-c}(1-2 c)\right)}{\gamma_{2} s e^{-c}+1}=\frac{d}{d t}\left(\frac{\left(\gamma_{1}+\gamma_{2}\right)\left(1-e^{-c}(1-2 c)\right) s^{2}}{2 c\left(1+\gamma_{2} s e^{-c}\right)}\right)
$$


Como

$$
u_{x}=-\frac{\left(\gamma_{1}+\gamma_{2}\right) e^{c\left(1-x^{2} / s^{2}\right)}\left(s^{2}-2 c x^{2}\right)}{s^{2}\left(e^{c}+\gamma_{2} s\right)}
$$

tem-se que

$$
u_{x}(s, t)=\frac{\left(\gamma_{1}+\gamma_{2}\right)(1-2 c)}{\left(e^{c}+\gamma_{2} s\right)}
$$

Substituindo na condição de Stefan, resulta

$$
\frac{d s}{d t}=\frac{\left(\gamma_{1}+\gamma_{2}\right) e^{-c}(1-2 c)}{\beta\left(1+\gamma_{2} s e^{-c}\right)}
$$

Para determinar uma condição inicial para $c$, basta notar que, à medida que $t \rightarrow 0$ e $s \rightarrow 0$, a temperatura $u$ tende a 0 e $u_{x} \rightarrow-\gamma_{1}-\gamma_{2}$ em $x=0$. A condição de Stefan então pode ser reescrita como

$$
-\beta \frac{d s}{d t} \rightarrow-\gamma_{1}-\gamma_{2}
$$

Logo, uma condição apropriada para $c$ seria $c(0)=0$. Mitchell e Myers (2010a) notam algumas singularidades próximas a $t=0$, e portanto seguem o estudo de Goodman (1958), incorporando uma nova variável ao problema. Faz-se uso de $\phi(t)=\int_{0}^{s} u d x$. Esta variável nada mais é que o lado direito da equação integral, e sua imediata condição inicial é $\phi(0)=0$. Agora tem-se um par de equações diferenciais em $s$ e $\phi$, uma vez que $c$ pode ser escrito em termos dessas variáveis. O sistema é fechado com a relação

$$
-2 c\left(1+\gamma_{2} e^{-c} s\right) \phi=\left(\gamma_{1}+\gamma_{2}\right)\left(1-e^{-c}(2 c+1)\right) s^{2}
$$

\subsubsection{O método de Balanceamento Integral Refinado}

O método de Balanceamento Integral Refinado (RIM) faz uso de uma segunda integração da equação diferencial. A diferença entre o método para problemas termais e o método para problemas de Stefan está justamente no uso da condição de Stefan.

$$
\int_{0}^{s}\left(\int_{0}^{x} \frac{\partial u}{\partial t} d \xi\right) d x=\int_{0}^{s}\left(\frac{\partial u}{\partial t}-\frac{\partial u}{\partial t}\right) d x=\left(\left.u\right|_{x=s}-\left.u\right|_{x=0}\right)-\left.s \frac{\partial u}{\partial x}\right|_{x=0}
$$

Já a integral do lado esquerdo pode ser calculada por partes

$$
\int_{0}^{s} \int_{0}^{x} \frac{\partial u}{\partial t} d \xi d x=s \int_{0}^{s} \frac{\partial u}{\partial t} d x-\int_{0}^{s} x \frac{\partial u}{\partial t} d x=s \frac{d}{d t} \int_{0}^{s} u d x-\frac{d}{d t} \int_{0}^{s} x u d x
$$

E portanto

$$
s \frac{d}{d t} \int_{0}^{s} u d x-\frac{d}{d t} \int_{0}^{s} x u d x=-\left.u\right|_{x=0}-\left.s \frac{\partial u}{\partial x}\right|_{x=0}
$$

Usando a equação do método HBIM 4.13,

$$
\begin{gathered}
s\left(\left.\frac{\partial u}{\partial x}\right|_{x=s}-\left.\frac{\partial u}{\partial x}\right|_{x=0}\right)-\frac{d}{d t} \int_{0}^{s} x u d x=-\left.u\right|_{x=0}-\left.s \frac{\partial u}{\partial x}\right|_{x=0} \\
\left.s \frac{\partial u}{\partial x}\right|_{x=s}+\left.u\right|_{x=0}=\frac{d}{d t} \int_{0}^{s} x u d x
\end{gathered}
$$


ou seja,

$$
\frac{d}{d t} \int_{0}^{s} x u d x=\left.u\right|_{x=0}-\beta s \frac{d s}{d t}
$$

Novamente, o método pode ser aplicado para problemas de condição de fronteira com temperatura constante ou uma condição de Robin, e com perfis de aproximação quadráticos, cúbicos ou exponenciais.

\section{Temperatura constante na fronteira}

A equação se torna

$$
\frac{d}{d t} \int_{0}^{s} x u d x=1-\beta s \frac{d s}{d t}
$$

Aproximando $u$ por um perfil quadrático

$$
u(x, t)=a\left(1-\frac{x}{s}\right)+(1-a)\left(1-\frac{x}{s}\right)^{2}
$$

tem-se

$$
\int_{0}^{s} x u d x=a \frac{s^{2}}{6}+(1-a) \frac{s^{2}}{12}=\frac{(a+1) s^{2}}{12}
$$

A equação integral se torna

$$
\frac{d}{d t}\left(\frac{(a+1) s^{2}}{12}\right)=1-\beta s \frac{d s}{d t}
$$

ou ainda

$$
s \frac{d s}{d t}=\frac{6}{1+a-6 \beta}
$$

e como pela condição de Stefan $s \frac{d s}{d t}=\frac{a}{\beta}$, tem-se

$$
\frac{a}{\beta}=\frac{6}{1+a-6 \beta}
$$

e portanto

$$
a^{2}+(1-6 \beta) a-6 \beta=0
$$

Como nesse caso supõe-se $a$ constante, tem-se

$$
s(t)=2 \alpha \sqrt{t}, \quad \alpha=\sqrt{\frac{a}{2 \beta}}
$$

e

$$
a=\frac{1}{2}\left(-(1+6 \beta)+\sqrt{1+36 \beta+36 \beta^{2}}\right)
$$

Para o perfil cúbico

$$
u(x, t)=a\left(1-\frac{x}{s}\right)+\frac{a^{2}}{2 \beta}\left(1-\frac{x}{s}\right)^{2}+\left(1-a-\frac{a^{2}}{2 \beta}\right)\left(1-\frac{x}{s}\right)^{3}
$$

tem-se

$$
\int_{0}^{s} x u d x=\frac{a s^{2}}{6}+\frac{a^{2} s^{2}}{24 \beta}-\frac{s^{2}\left(a^{2}-2 \beta+2 a \beta\right)}{40 \beta}
$$


A equação integral se torna

$$
\frac{d}{d t}\left(\frac{s^{2}\left(a^{2}+3 \beta+7 a \beta\right)}{60 \beta}\right)=1-\beta s \frac{d s}{d t}
$$

Como nesse caso supõe-se $a$ constante, tem-se

$$
s(t)=2 \alpha \sqrt{t}, \quad \alpha=\sqrt{\frac{a}{2 \beta}}
$$

onde $a$ é encontrado isolando o termo $\frac{d s}{d t}$ e igualando com a condição de Stefan, resolvendo a equação

$$
a^{3}+7 \beta a^{2}+3 \beta(1+10 \beta) a-30 \beta^{2}=0
$$

Para o perfil exponencial, a solução continua sendo

$$
s=2 \alpha \sqrt{t}, \quad \alpha=\sqrt{\frac{1-2 c}{2 \beta}}
$$

mas a equação 4.21 se torna

$$
(1-2 c)\left(2 \sqrt{c}+2 c^{3 / 2}-\sqrt{\pi} e^{c} \operatorname{erf}(\sqrt{c})\right)=4 \beta c^{5 / 2}
$$

\subsubsection{O problema de Stefan imobilizado}

A técnica de imobilização de fronteira apresentada no capítulo anterior também pode ser estendida para lidar com problemas de Stefan de uma fase. Aqui será utilizado, supondo $m$ e $a$ constantes, o perfil polinomial genérico

$$
u=a\left(1-\frac{x}{s}\right)+(1-a)\left(1-\frac{x}{s}\right)^{m}
$$

Lembrando a definição da função $f$ do capítulo 2

$$
f(x, t)=\frac{\partial u}{\partial t}-\frac{\partial^{2} u}{\partial x^{2}}
$$

Então, para este perfil aproximado tem-se

$$
f(x, t)=\frac{(1-a) m x s_{t}}{s^{2}}\left(1-\frac{x}{s}\right)^{m-1}+\frac{a x s_{t}}{s^{2}}-\frac{(1-a)(m-1) m}{s^{2}}\left(1-\frac{x}{s}\right)^{m-2}
$$

A partir dessa equação, pode-se ver que $f(0, t)$ só será zero se $a=1$, mas esse é o perfil linear, que normalmente provê uma aproximação pobre. Em $x=s$, encontra-se $f(s, t) \rightarrow \infty$ se $m<2$. Para $m \geq 2, f(s, t)$ é finito, mas nunca zero. O erro definido por Langford (1973) é novamente singular em $t=0$.

Nos moldes da imobilização de fronteira do capítulo anterior, será definida agora a mudança de variáveis $\xi=\frac{x}{s(t)}$. É importante lembrar que naquele capítulo era utilizada a mudança $\xi=\frac{x}{\delta(t)}$; como já foi dito, a fronteira móvel assume o papel da profundidade de penetração. Isso transforma 
o problema em

$$
\begin{array}{r}
\frac{\partial U}{\partial t}-\frac{s_{t}}{s} \xi \frac{\partial U}{\partial \xi}=\frac{1}{s^{2}} \frac{\partial^{2} U}{\partial \xi^{2}}, \quad 0<\xi<1 \\
U(0, t)=1, \\
U(1, t)=0, \\
\beta s s_{t}=-\left.\frac{\partial U}{\partial \xi}\right|_{\xi=1}
\end{array}
$$

O perfil de aproximação se torna

$$
U=a(1-\xi)+(1-a)(1-\xi)^{m}
$$

e a função $F$, como definida em 3.15

$$
F(\xi, t)=-s s_{t} \xi \frac{\partial U}{\partial \xi}-\frac{\partial^{2} U}{\partial \xi^{2}}
$$

O erro é definido $E_{M}=\int_{0}^{1} F^{2} d \xi$ ou

$$
\begin{aligned}
E_{M} & =\int_{0}^{1}\left(-s s_{t} \xi \frac{\partial U}{\partial \xi}-\frac{\partial^{2} U}{\partial \xi^{2}}\right)^{2} d \xi \\
& =\int_{0}^{1}\left(-s s_{t} \xi\left(-a-m(1-a)(1-\xi)^{m-1}\right)-\left(m(m-1)(1-a)(1-\xi)^{m-2}\right)\right)^{2} d \xi \\
& =\int_{0}^{1}\left(-\frac{a}{\beta} \xi\left(-a-m(1-a)(1-\xi)^{m-1}\right)-\left(m(m-1)(1-a)(1-\xi)^{m-2}\right)\right)^{2} d \xi
\end{aligned}
$$

Para chegar a essa equação foi necessário o uso da condição de Stefan que nesse caso se reduz a $s s_{t}=\frac{a}{\beta}$; É evidente que $E_{M}$ é independente do tempo. Porém, é claro que o erro depende do valor $\beta$ e também o valor ótimo de $m$ varia $\operatorname{com} \beta$.

As formulações dos métodos HBIM e RIM são dadas por $\int_{0}^{1} F d \xi=0$ e $\int_{0}^{1} \xi F d \xi=0$ como no capítulo 3 , e resultam respectivamente em

$$
s s_{t} \int_{0}^{1} U d \xi=\left.\frac{\partial U}{\partial \xi}\right|_{\xi=1}-\left.\frac{\partial U}{\partial \xi}\right|_{\xi=0}, \quad 2 s s_{t} \int_{0}^{1} \xi U d \xi=1+\left.\frac{\partial U}{\partial \xi}\right|_{\xi=1}
$$

Notando que

$$
\int U d \xi=-a \frac{(1-\xi)^{2}}{2}-(1-a) \frac{(1-\xi)^{m+1}}{m+1}
$$

que

$$
\frac{\partial U}{\partial \xi}=-a-m(1-a)(1-\xi)^{m-1}
$$

e que

$$
\int \xi U d \xi=-\frac{(a-1)(\xi-1)(m \xi+\xi+1)(1-\xi)^{m}}{(m+1)(m+2)}-\frac{a \xi^{3}}{3}+\frac{a \xi^{2}}{2}
$$

as formulações dos métodos resultam em

$$
s s_{t}=\frac{2 m(m+1)(1-a)}{a(m+1)+2}
$$




$$
s s_{t}=\frac{3(1-a)(m+1)(m+2)}{a(m-1)(m+4)+6}
$$

para os métodos HBIM e RIM respectivamente.

Igualando essas duas equações, tem-se o valor de $a$

$$
a=\frac{6(2-m)}{(m-1)\left(2 m^{2}+5 m-6\right)}
$$

E substituindo em uma das equações unidas com a condição de Stefan $s s_{t}=\frac{a}{\beta}$, tem-se a equação para $m$

$$
\beta(2 m-3)(2+m)(1+m)\left(2 m^{2}+5 m-6\right)+12(m-2)=0
$$

\subsubsection{Refinamento usando subdivisões espaciais - o método Nodal}

A abordagem de refinamento que utiliza subdivisões espaciais pode ser determinada de duas maneiras. A primeira, proposta por Mosally et al. (2005), sugere perfis de aproximações lineares em cada subdivisão espacial, de maneira similar ao que foi feito na seção 2.2.4, mas agora para problemas de Stefan. Mosally demonstra ainda em seu trabalho propriedades da convergência do esquema nodal, quando $n \rightarrow \infty$. Sua análise destaca as propriedades de convergência lentas do método básico com perfil linear $\left(O\left(n^{-1}\right)\right.$ para $\beta$ grande). O trabalho de Mosally et al. procura generalizar para problemas de Stefan de uma fase o trabalho de Bell e Abbas (1985), que investigava a convergência do método de subdivisões espaciais para o problema termal.

A segunda maneira, apresentada por Mitchell (2011), é novamente uma aplicação direta do que foi feito na seção 3.3 para problemas termais. A imobilização da fronteira ainda é a mudança de variáveis $\xi=\frac{x}{s(t)}$.

\section{Solução HBI linear por partes}

Para aproximar a solução do problema de Stefan o intervalo $[0, s]$ é subdividido em $n$ células iguais de tamanho $s / n$ e a temperatura $u$ é aproximada em cada nó $x_{i}=i \frac{s}{n}$ por $u_{i}=u\left(x_{i}, t\right)$, com $u_{0}=1$ e $u_{n}=0$. O perfil de aproximação linear é escolhido (exatamente como 2.15)

$$
f(x)=u_{i}+\left(u_{i+1}-u_{i}\right) \frac{\left(x-x_{i}\right)}{x_{i+1}-x_{i}}, \quad i=0, \ldots, n-1
$$

Reescrevendo a equação 2.17 , mas com $s(t)$ no lugar de $\delta(t)$

$$
s \frac{d s}{d t}=-\frac{2 n^{2}}{2 i+1} \frac{u_{i+2}-2 u_{i+1}+u_{i}}{u_{i+1}-u_{i}}, \quad i=0 \ldots n-2
$$

e para $i=n-1$, tem-se

$$
-\frac{(2 n-1)\left(u_{n}-u_{n-1}\right)}{2 n} \frac{d s}{d t}=-\beta \frac{d s}{d t}-n \frac{u_{n}-u_{n-1}}{s}
$$

ou seja,

$$
s \frac{d s}{d t}=\frac{2 n^{2} u_{n-1}}{(2 n-1) u_{n-1}+2 n \beta}
$$


Observe que

$$
\begin{aligned}
-\frac{\left(2 n^{2}\right)\left(u_{i+2}-2 u_{i+1}+u_{i}\right)}{(2 i+1)\left(u_{i+1}-u_{i}\right)} & =\frac{2 n^{2} u_{n-1}}{2 \beta n+(2 n-1) u_{n-1}} \\
-\frac{1}{2 i+1}\left(\frac{u_{i+2}-u_{i+1}}{u_{i+1}-u_{i}}-1\right) & =\frac{u_{n-1}}{2 \beta n+(2 n-1) u_{n-1}} \\
\frac{u_{i+2}-u_{i+1}}{u_{i+1}-u_{i}}-1 & =-\frac{(2 i+1) u_{n-1}}{2 \beta n+(2 n-1) u_{n-1}} \\
\frac{u_{i+2}-u_{i+1}}{u_{i+1}-u_{i}} & =1-\frac{(2 i+1) u_{n-1}}{2 \beta n+(2 n-1) u_{n-1}} \\
\frac{u_{i+2}-u_{i+1}}{u_{i+1}-u_{i}} & =\frac{-(2 i+1) u_{n-1}+2 \beta n+(2 n-1) u_{n-1}}{2 \beta n+(2 n-1) u_{n-1}} \\
\frac{u_{i+2}-u_{i+1}}{u_{i+1}-u_{i}} & =\frac{(-2 i+2 n-1-1) u_{n-1}+2 \beta n}{2 \beta n+(2 n-1) u_{n-1}} \\
\frac{u_{i+2}-u_{i+1}}{u_{i+1}-u_{i}} & =\frac{(-i+n-1) u_{n-1}+\beta n}{\beta n+\left(n-\frac{1}{2}\right) u_{n-1}} \\
\left(u_{i+2}-u_{i+1}\right) \frac{\left(n-\frac{1}{2}\right) u_{n-1}+\beta n}{(n-i-1) u_{n-1}+\beta n} & =u_{i+1}-u_{i}
\end{aligned}
$$

e portanto

$$
u_{i}=u_{i+1}-\left(u_{i+2}-u_{i+1}\right) \frac{\left(n-\frac{1}{2}\right) u_{n-1}+\beta n}{(n-i-1) u_{n-1}+\beta n}
$$

Com essas equações tem-se um sistema de $n-1$ equações não-lineares e $n-1$ valores para as temperaturas $u_{1}, \ldots, u_{n-1}$, que pode ser reescrito como

$$
u_{i}=u_{i+1}-\left(u_{i+2}-u_{i+1}\right) g_{i}, \quad i=n-2, n-3, \ldots, 0
$$

e

$$
g_{i}=\frac{n+\eta-1 / 2}{n+\eta-i-1}, \quad \eta=\frac{n \beta}{u_{n-1}}
$$

Assim como pode ser feito no método nodal para problemas termais da seção 2.2.4, pode-se escrever, através de substituição reversa,

$$
u_{i}=u_{n-1}\left(1+\sum_{l=i}^{n-2} \prod_{j=l}^{n-2} g_{j}\right)=u_{n-1} \sum_{l=i}^{n-1} \frac{(n+\eta-1 / 2)^{n-l-1}}{(n+\eta-l-1) !} \eta !=u_{n-1} \sum_{k=0}^{n-i-1} \frac{(n+\eta-1 / 2)^{k}}{(\eta+k) !} \eta !
$$

Como $u_{0}=1$, tem-se

$$
u_{n-1} \sum_{k=0}^{n-1} \frac{(n+\eta-1 / 2)^{k}}{(\eta+k) !} \eta !=1
$$

E eliminando o termo $u_{n-1}$ das equações anteriores, tem-se uma aproximação para $u_{i}$ dependendo de $n, \eta$ e $i$

$$
u_{i}=\frac{\sum_{k=0}^{n-i-1} \frac{(n+\eta-1 / 2)^{k}}{(\eta+k) !}}{\sum_{k=0}^{n-1} \frac{(n+\eta-1 / 2)^{k}}{(\eta+k) !}}, \quad i=0, \ldots, n-1
$$

Essa fórmula é análoga à fórmula obtida por Bell e Abbas (1985) para aproximar a solução do problema termal, exibida na seção 2.2.4. A novidade é o valor $\eta$, que é uma função não-linear de $n$ que captura o efeito da frente móvel $s$, que se move com velocidade finita. Para encontrar o valor 
de $\eta$, basta substituir na equação 4.30 , o valor de $u_{n-1}$ por $n \beta / \eta$, e resolver a equação. Uma vez que $\eta$ é determinado e os $u_{i}$ 's são encontrados, $s$ é encontrado como a solução da equação 4.29:

$$
s(t)=2 \frac{n}{\sqrt{2 n+2 \eta-1}} \sqrt{t}=2 \alpha^{*} \sqrt{t}
$$

\section{Imobilização de Fronteira e Subdivisões Espaciais}

Dado o problema de Stefan unidimensional de uma fase com condição de fronteira dependente do tempo

$$
\begin{aligned}
\frac{\partial^{2} u}{\partial x^{2}} & =\frac{\partial u}{\partial t}, \quad 0<x<s(t) \\
\beta \frac{d s}{d t} & =-\frac{\partial u}{\partial x}, \quad \text { em } x=s \\
u & =0, \quad \text { em } x=s \\
u(x, 0) & =0, \quad \text { para } x>0 \\
u(0, t) & =h(t)
\end{aligned}
$$

Para imobilizar a fronteira faz-se

$$
\xi=\frac{x}{s(t)}, \quad u(x, t)=h(t) U(\xi, t)
$$

E o problema se torna

$$
\begin{aligned}
h U_{\xi \xi} & =s^{2} h_{t} U+s^{2} h U_{t}-\xi s s_{t} h U_{\xi}, \quad 0<\xi<1 \\
\beta s s_{t} & =-h \frac{\partial U}{\partial \xi}, \quad \text { em } \xi=1 \\
U & =0, \quad \text { em } \xi=1 \\
U(\xi, 0) & =0, \quad \text { para } \xi>0 \\
U(0, t) & =1
\end{aligned}
$$

Considerando novamente o perfil cúbico da seção 3.3, com as condições 3.17 e 3.18 tal que $f_{i}\left(x_{i+1}\right)=U_{i+1}$ e a primeira e segunda derivadas são correspondidas em cada ponto nodal. Então, a equação de balanceamento integral leva ao sistema 3.21, mas com $\delta=s$. Para o problema termal foram obtidas duas outras equações que requeriam $U_{\xi}(1, t)=U_{\xi \xi}(1, t)=0$. Essas duas equações não se aplicam no caso atual, mas pode-se utilizar a condição de Stefan para deduzir

$$
\beta s s_{t}=-n h\left(\alpha_{n-1}+2 \beta_{n-1}+3 \gamma_{n-1}\right)
$$

Existe, agora uma escolha em como especificar a condição final. Uma formulação de spline cúbica normalmente exige que a segunda derivada seja zero na fronteira esquerda, isso é, $\beta_{0}=0$. Contudo, para ser mais consistente com o problema termal, segue-se como Goodman (1958) que faz a abordagem da derivada total, mas agora em $s(t)$ :

$$
\frac{D u}{D t}(s, t)=\left.\left(\frac{\partial u}{\partial t}+\frac{\partial u}{\partial x} \frac{d s}{d t}\right)\right|_{x=s}=0 \Rightarrow \beta \frac{\partial^{2} u}{\partial x^{2}}=\left.\left(\frac{\partial u}{\partial x}\right)\right|_{x=s}
$$


que depois da mudança de variáveis fica

$$
\beta \frac{\partial^{2} U}{\partial \xi^{2}}=\left.h\left(\frac{\partial U}{\partial \xi}\right)^{2}\right|_{\xi=1}
$$

e portanto

$$
\beta\left(2 \beta_{n-1}+6 \gamma_{n-1}\right)=h\left(\alpha_{n-1}+2 \beta_{n-1}+3 \gamma_{n-1}\right)^{2}
$$

Não confundir $\beta$ o número de Stefan $\operatorname{com} \beta_{n-1}$ o coeficiente do perfil cúbico de aproximação.

\subsection{Outras Aproximações Numéricas}

Outros métodos de aproximação da solução do problema de Stefan incluem os métodos de diferenças finitas Variable Space Grid (Malha de Espaçamento Variável) e seu equivalente de fronteira imobilizada Boundary Immobilization Method. Uma variedade de técnicas numéricas para problemas de Stefan pode ser encontrada no compêndio de Crank (1987).

\subsubsection{O método Variable Space Grid (VSG)}

Aqui será analisado o problema de Stefan com condição de fronteira de fluxo constante. A quantidade de intervalos espaciais entre um domínio fixo $(x=0)$ e um domínio móvel $(x=s(t))$ foi mantida constante e igual a $n$ de modo que a fronteira móvel sempre esteja na n-ésima posição da malha. $\mathrm{O}$ aspecto mais importante do método é o fato de o tamanho da malha $\Delta x=s(t) / n$ variar com o tempo $t$ em cada passo de tempo $\Delta t$, dado que existe um número fixado de pontos. Para o i-ésimo ponto da malha, vale

$$
\frac{D u}{D t}\left(x_{i}, t\right)=\frac{\partial u}{\partial x}\left(x_{i}, t\right) x_{i}^{\prime}+\frac{\partial u}{\partial t}\left(x_{i}, t\right)
$$

e o nó $x_{i}$ se move de acordo com a expressão

$$
\frac{d x}{d t}=\frac{x_{i}}{s(t)} \frac{d s}{d t}
$$

Portanto, tem-se a equação nos pontos da malha

$$
u_{t}\left(x_{i}, t\right)=\frac{x_{i}}{s} \frac{d s}{d t} u_{x}\left(x_{i}, t\right)+u_{x x}\left(x_{i}, t\right)
$$

com as condições

$$
u\left(x_{0}, t\right)=1, \quad u\left(x_{n}, t\right)=0
$$

e a condição de Stefan

$$
\beta \frac{d s}{d t}(t)=-\frac{\partial u}{\partial x}\left(x_{n}, t\right)
$$

Uma solução numérica explícita baseada em diferenças finitas para o problema acima é facilmente obtida ao substituir as derivadas no tempo e no espaço nos nós $\left(x_{i}^{m}, t_{m}\right)$ pelas diferenças progressivas e centradas usuais respectivamente. As primeiras derivadas com respeito à variável tempo são representadas por diferenças progressivas. Para a derivada na fronteira móvel, Furzeland 
(1980) sugere a diferença regressiva de três termos:

$$
\left.\frac{\partial u}{\partial x}\right|_{x=s}={ }^{\beta} \frac{3 u_{n}-4 u_{n-1}+u_{n-2}}{2 \Delta x}+O\left(\Delta x^{2}\right)
$$

Tem-se a seguinte aproximação explícita via diferenças finitas para a equação 4.36

$$
u_{i}^{m+1}=u_{i}^{m}+\frac{k x_{i}^{m}}{2 h} \frac{s_{m}^{\prime}}{s_{m}}\left(u_{i+1}^{m}-u_{i-1}^{m}\right)+\frac{k}{h^{2}}\left(u_{i+1}^{m}-2 u_{i}^{m}+u_{i-1}^{m}\right)
$$

com erro de truncamento de $O(k)+O\left(h^{2}\right)$. Nas equações acima tem-se $u_{i}^{m} \sim U\left(x_{i}^{m}, t_{m}\right), x_{i}^{m}=i h_{m}$, $t_{m}=t_{0}+m k$. Note também que $h_{m}=\Delta x^{m}$ é o tamanho do grid no m-ésimo passo de tempo, e $k$ é o passo de tempo; $t_{0}$ é um tempo inicial.

A condição de fronteira em $x=0$, em termos de diferenças centradas, pode ser escrita como

$$
u_{-1}^{m}=u_{1}^{m}-2 h
$$

Em $i=0$, a equação 4.37 também exibe o termo $u_{-1}^{m}$. Assim, pode-se utilizar o termo vindo da condição de fronteira em $x=0$.

O esquema numérico pode, então, ser escrito na forma explícita para $m=0,1,2, \ldots$ como

$$
\begin{aligned}
& u_{0}^{m+1}=u_{0}^{m}\left(1-\frac{2 k}{h^{2}}\right)+u_{1}^{m} \frac{2 k}{h^{2}}+\left(k x_{0}^{m} \frac{s_{m}^{\prime}}{s_{m}}-\frac{2 k}{h}\right) \\
& u_{i}^{m+1}=u_{i}^{m}+\frac{k x_{i}^{m}}{2 h} \frac{s_{m}^{\prime}}{s_{m}}\left(u_{i+1}^{m}-u_{i-1}^{m}\right)+\frac{k}{h^{2}}\left(u_{i+1}^{m}-2 u_{i}^{m}+u_{i-1}^{m}\right), \quad i=1, \ldots, n-1 \\
& u_{N}^{m}=0
\end{aligned}
$$

unido ao esquema para a fronteira

$$
\begin{array}{r}
s_{m+1}=s_{m}-\beta \frac{k}{2 h}\left(3 u_{n}^{m}-4 u_{n-1}^{m}+u_{n-2}^{m}\right), \quad m=0,1,2, \ldots \\
s_{0}=0
\end{array}
$$

A cada passo de tempo o tamanho do grid $h_{m+1}$ é recalculado, $h_{m+1}=s_{m+1} / n$. O seguinte limite no tamanho do passo de tempo $k$ é apresentado após análise de estabilidade de Von Neumann no trabalho de Kutluay et al. (1997)

$$
k \leq \frac{2 h^{2}}{4+\left(h s^{\prime}\right)^{2}}
$$

\subsubsection{O método de fronteira imobilizada (BIM)}

Também é possível fixar a fronteira móvel utilizando-se a técnica de imobilização de fronteira apresentada. Novamente, a mudança de variáveis será $\xi=\frac{x}{s(t)}$. O problema imobilizado se torna

$$
\begin{array}{r}
\frac{\partial U}{\partial t}-\frac{s_{t}}{s} \xi \frac{\partial U}{\partial \xi}=\frac{1}{s^{2}} \frac{\partial^{2} U}{\partial \xi^{2}}, \quad 0<\xi<1 \\
U_{\xi}(0, t)=-1 \\
U(1, t)=0
\end{array}
$$




$$
\beta s s_{t}=-\left.\frac{\partial U}{\partial \xi}\right|_{\xi=1}
$$

O esquema numérico imobilizado também pode ser escrito na forma explícita como

$$
\begin{array}{r}
u_{0}^{m+1}=u_{0}^{m}\left(1-\frac{2 k}{h^{2} s_{m}^{2}}\right)+u_{1}^{m} \frac{2 k}{h^{2} s_{m}^{2}}+\left(k \xi_{0} \frac{s_{m}^{\prime}}{s_{m}}+\frac{2 k}{s_{m}^{2}}\right) \\
u_{i}^{m+1}=u_{i}^{m}+\frac{k \xi_{i}}{2 h} \frac{s_{m}^{\prime}}{s_{m}}\left(u_{i+1}^{m}-u_{i-1}^{m}\right)+\frac{k}{h^{2} s_{m}^{2}}\left(u_{i+1}^{m}-2 u_{i}^{m}+u_{i-1}^{m}\right), \\
i=1, \ldots, n-1 \\
u_{N}^{m}=0
\end{array}
$$

unido ao esquema para a fronteira

$$
\begin{array}{r}
s_{m+1}=s_{m}-\beta \frac{k}{2 h}\left(3 u_{n}^{m}-4 u_{n-1}^{m}+u_{n-2}^{m}\right), \quad m=0,1,2, \ldots \\
s_{0}=0
\end{array}
$$

Por fim, com a análise de estabilidade de Von Neumann obtém-se um limitante para o passo de tempo k:

$$
k \leq \frac{1}{2} h^{2} s^{2}
$$




\section{Capítulo 5}

\section{Resultados e Considerações Finais}

No presente trabalho foi mostrado como o método de balanceamento integral, em suas diversas formas, pode ser aplicado a problemas termais e problemas de Stefan. Alguns autores criticam a não existência de uma maneira padronizada de se verificar a precisão de soluções, mas o recente trabalho de Mitchell e Myers (2012) procura estabelecer critérios para o erro das aproximações ao rever os trabalhos de Langford (1973) sob a ótica de imobilização de fronteira. Essa nova maneira de olhar o erro não requer o conhecimento prévio de uma solução exata. A análise aqui é feita também através da tradicional observação da porcentagem de erro em $\alpha$ e do erro máximo em um instante de tempo, tipicamente $t=1$.

\section{Precisão do HBIM e do RIM em problemas de Stefan}

\begin{tabular}{rrrrrrrr}
\multicolumn{3}{c}{ HBIM } & & \multicolumn{3}{c}{ RIM } \\
\hline$\beta$ & $\alpha$ real & Quadrático & Cúbico & Exponencial & Quadrático & Cúbico & Exponencial \\
\hline 0.75 & 0.692456 & 0.714134 & 0.698096 & 0.689812 & 0.694315 & 0.692993 & 0.691977 \\
1.00 & 0.620063 & 0.636494 & 0.623513 & 0.618572 & 0.621290 & 0.620335 & 0.619839 \\
1.25 & 0.566925 & 0.579921 & 0.569224 & 0.565986 & 0.567781 & 0.56708 & 0.566805 \\
2.50 & 0.421238 & 0.426974 & 0.421814 & 0.421032 & 0.421472 & 0.42126 & 0.421222 \\
5.00 & 0.306424 & 0.308722 & 0.306549 & 0.306383 & 0.306477 & 0.306427 & 0.306422 \\
10.0 & 0.220016 & 0.220885 & 0.220041 & 0.220009 & 0.220027 & 0.220017 & 0.220016 \\
\hline
\end{tabular}

Tabela 5.1: $\alpha$ aproximado para distintos valores de $\beta$ em problemas de Stefan com condição de temperatura constante na fronteira

\begin{tabular}{rrrrrrr}
\multicolumn{3}{c}{ HBIM } & & \multicolumn{2}{c}{ RIM } \\
\hline$\beta$ & Quadrático & Cúbico & Exponencial & Quadrático & Cúbico & Exponencial \\
\hline 0.75 & $3.04 \mathrm{E}-02$ & $8.08 \mathrm{E}-03$ & $3.83 \mathrm{E}-03$ & $2.68 \mathrm{E}-03$ & $7.75 \mathrm{E}-04$ & $6.92 \mathrm{E}-04$ \\
1.0 & $2.58 \mathrm{E}-02$ & $5.53 \mathrm{E}-03$ & $2.41 \mathrm{E}-03$ & $1.97 \mathrm{E}-03$ & $4.38 \mathrm{E}-04$ & $3.61 \mathrm{E}-04$ \\
1.25 & $2.24 \mathrm{E}-02$ & $4.04 \mathrm{E}-03$ & $1.66 \mathrm{E}-03$ & $1.51 \mathrm{E}-03$ & $2.73 \mathrm{E}-04$ & $2.12 \mathrm{E}-04$ \\
2.5 & $1.34 \mathrm{E}-02$ & $1.37 \mathrm{E}-03$ & $4.89 \mathrm{E}-04$ & $5.55 \mathrm{E}-04$ & $5.22 \mathrm{E}-05$ & $3.80 \mathrm{E}-05$ \\
5.0 & $7.44 \mathrm{E}-03$ & $4.08 \mathrm{E}-04$ & $1.34 \mathrm{E}-04$ & $1.73 \mathrm{E}-04$ & $9.79 \mathrm{E}-06$ & $6.53 \mathrm{E}-06$ \\
10.0 & $3.93 \mathrm{E}-03$ & $1.14 \mathrm{E}-04$ & $3.18 \mathrm{E}-05$ & $5.00 \mathrm{E}-05$ & $4.55 \mathrm{E}-06$ & $0.00 \mathrm{E}+00$ \\
\hline
\end{tabular}

Tabela 5.2: erro percentual de $\alpha$ aproximado com relação a $\alpha$ real para distintos valores de $\beta$ em problemas de Stefan com condição de temperatura constante na fronteira

As tabelas apresentadas mostram para os perfis quadráticos, cúbicos e exponenciais apresentados 
no capítulo 4, os $\alpha$ encontrados por cada método e as porcentagens de erro de cada $\alpha$. Para o HBIM, o perfil exponencial e o perfil cúbico apresentam erros de menor magnitude que os erros do perfil quadrático. É possivel observar que o perfil exponencial é melhor para todos os valores de $\beta$. Olhando para o método RIM, existe uma melhora na magnitude do erro em todos os perfis. Novamente o exponencial apresenta o melhor resultado, mas os erros são muito similares ao perfil cúbico. Porém, Mitchell e Myers (2010a) nota que isso vale apenas para condição de temperatura constante na fronteira. Isso não é surpresa, pois o perfil exponencial foi desenvolvido baseado na solução exata. A tabela a seguir foi retirada de Mitchell e Myers (2010a) a fim de ilustrar essa situação. O perfil cúbico do HBIM é melhor em quase todos os casos, exceto para $\beta=1$.

\begin{tabular}{rrrrrrr}
\multicolumn{3}{c}{ HBIM } & & \multicolumn{3}{c}{ RIM } \\
\hline$\beta$ & Quadrático & Cúbico & Exponencial & Quadrático & Cúbico & Exponencial \\
\hline 1 & $7.8 \mathrm{E}-03$ & $1.5 \mathrm{E}-03$ & $1.1 \mathrm{E}-02$ & $4.2 \mathrm{E}-03$ & $1.3 \mathrm{E}-03$ & $7.7 \mathrm{E}-03$ \\
1.25 & $5.5 \mathrm{E}-03$ & $8.6 \mathrm{E}-04$ & $8.3 \mathrm{E}-03$ & $3.6 \mathrm{E}-03$ & $9.0 \mathrm{E}-04$ & $6.6 \mathrm{E}-03$ \\
1.67 & $3.4 \mathrm{E}-03$ & $3.8 \mathrm{E}-04$ & $5.4 \mathrm{E}-03$ & $2.6 \mathrm{E}-03$ & $4.7 \mathrm{E}-04$ & $5.3 \mathrm{E}-03$ \\
2.5 & $1.5 \mathrm{E}-03$ & $1.0 \mathrm{E}-04$ & $2.7 \mathrm{E}-03$ & $1.7 \mathrm{E}-03$ & $1.6 \mathrm{E}-04$ & $3.7 \mathrm{E}-03$ \\
5 & $3.0 \mathrm{E}-04$ & $6.0 \mathrm{E}-06$ & $5.8 \mathrm{E}-04$ & $4.3 \mathrm{E}-04$ & $2.3 \mathrm{E}-04$ & $1.8 \mathrm{E}-03$ \\
10 & $4.2 \mathrm{E}-05$ & $2.0 \mathrm{E}-06$ & $5.0 \mathrm{E}-04$ & $2.4 \mathrm{E}-04$ & $2.9 \mathrm{E}-04$ & $8.2 \mathrm{E}-03$ \\
\hline
\end{tabular}

Tabela 5.3: erro percentual de $\alpha$ aproximado em relação a a real para distintos valores de $\beta$ em problemas com condição de Robin

Para essa tabela, Mitchell e Myers (2010a) utilizou os resultados numéricos usando um método de diferenças finitas de Mitchell e Vynnycky (2009), uma vez que não há solução exata para este problema. Os erros do perfil cúbico do RIM são similares aos erros do perfil cúbico do HBIM.

\section{Vantagens do método nodal}

O objetivo do trabalho Mitchell (2011) foi desenvolver um framework geral para aproximar com eficácia a solução de problemas termais e de Stefan unidimensionais através do método de balanceamento integral nodal. A introdução da técnica com perfis de ordem maior que linear garante uma alta precisão nas soluções. Além disso, a técnica pode ser facilmente estendida para problemas com condição de fronteiras genéricas que dependem do tempo. Nesse trabalho ainda é destacado que o método nodal com perfis cúbicos parece suficiente para predizer uma solução, mesmo quando a condição de fronteira é monótona decrescente no tempo, ou oscilatória. O próprio Goodman (1958) notou que o método clássico de balanceamento integral é útil apenas para condições de fronteira dependentes do tempo se essas condições forem monótonas crescentes ou constantes.

$\mathrm{Na}$ figura 5.1 a seguir são exibidas as seguintes aproximações em $t=1$ para a solução do problema termal com condição de fronteira de temperatura constante:

(i) o método HBIM com $m=2$ (obtido através do CIM) em linha tracejada

(ii) o método nodal com perfil linear para $n=4 \mathrm{em}$ linha tracejada-pontilhada

(iii) o método nodal com perfil cúbico para $n=4$ subdivisões espaciais em linha contínua

(iv) a solução exata em linha contínua 
Nessa ilustração a solução está muito próxima da aproximação via método nodal com perfil cúbico, sendo impossível distingui-las. O RIM não é mencionado na imagem pois para esse caso ele coincide com o HBIM (uma vez que $\sqrt{2 m(m+1)}=\sqrt{(m+1)(m+2)}$, para $m=2$ ).

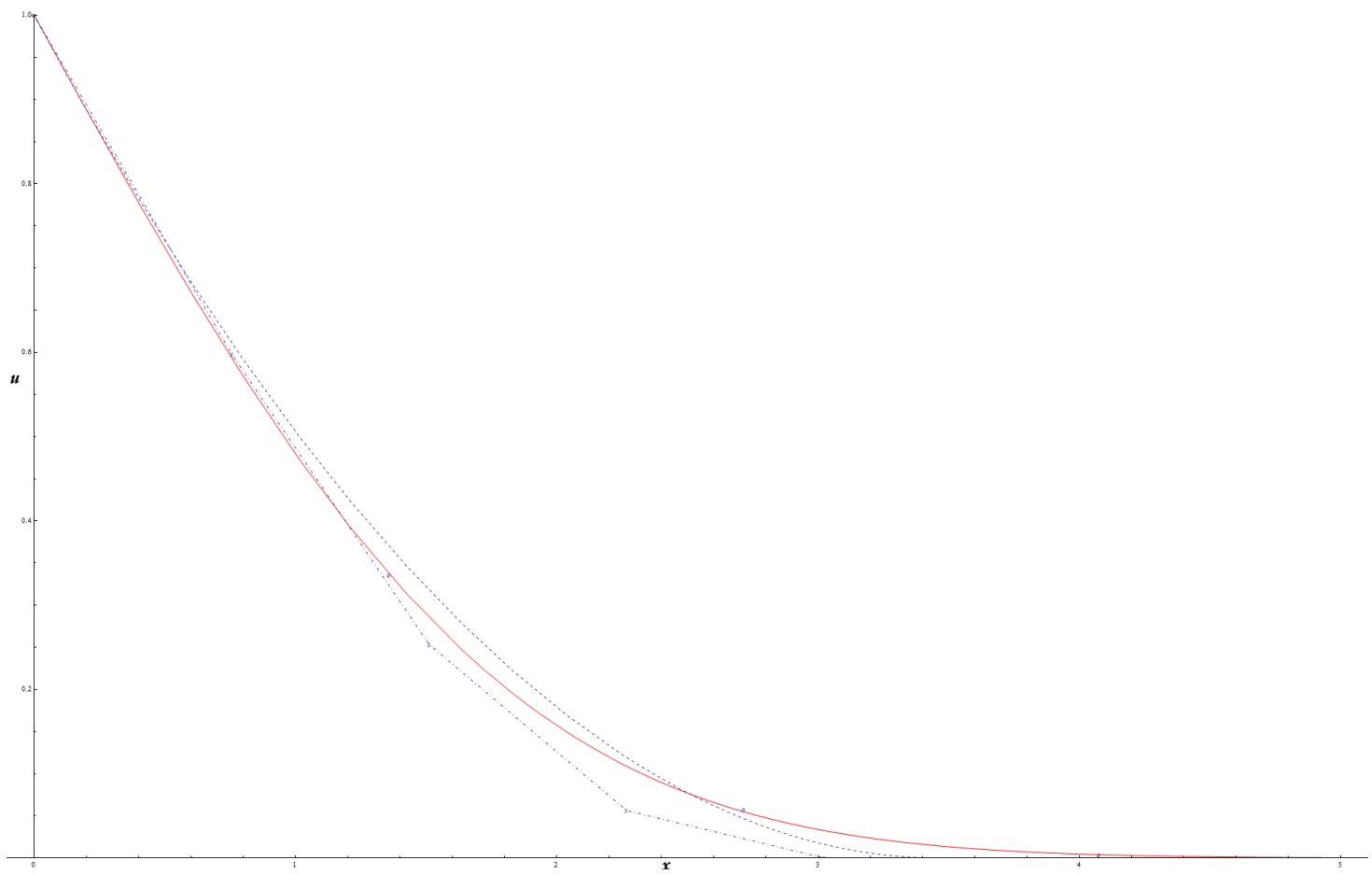

Figura 5.1: Gráfico da solução do problema 2.1 com temperatura constante na fronteira

\begin{tabular}{cc} 
Método & Erro máximo em $u(x, 1)$ \\
\hline Nodal Linear & 0.40702 \\
HBIM & $5.0 \mathrm{E} 10-3$ \\
Nodal Cúbico & $5.0 \mathrm{E} 10-4$
\end{tabular}

Tabela 5.4: Erro máximo em u $(x, 1)$ para aproximações de soluçôes do problema com temperatura constante na fronteira

Para problemas de Stefan, o método nodal se mostra mais preciso que o método CIM quando a condição de fronteira é constante. A tabela 5.5 mostra resultados para esse problema. Foi utilizado $n=4$ subdivisões espaciais para o perfil cúbico. É possível observar que o CIM é menos preciso que o método nodal nas três observações de $\beta$.

\begin{tabular}{ccccc}
\hline & \multicolumn{2}{c}{ Porcentagem de erro em $\alpha$} & \multicolumn{2}{c}{ Erro máximo para $u(x, 1)$} \\
\cline { 2 - 5 }$\beta$ & Cúbico & CIM & Cúbico & CIM \\
\hline 0.1 & $2.42 \times 10^{-2}$ & 2.161 & $2.08 \times 10^{-4}$ & $2.28 \times 10^{-2}$ \\
1 & $1.05 \times 10^{-3}$ & $2.52 \times 10^{-1}$ & $1.25 \times 10^{-5}$ & $9.22 \times 10^{-3}$ \\
10 & $2.89 \times 10^{-5}$ & $4.39 \times 10^{-3}$ & $2.80 \times 10^{-7}$ & $1.41 \times 10^{-3}$ \\
\hline
\end{tabular}

Tabela 5.5: Resultados para o problema de Stefan com temperatura constante na fronteira.

A análise apresentada em Mosally et al. (2005) para uma implementação com subdivisões espaciais e perfis lineares mostra uma convergência mais devagar que a observada em métodos de diferenças finitas. O trabalho de Mitchell (2011) não realiza análise similar, visto que busca, com 
seu método, uma aproximação de alta precisão com apenas poucos pontos nodais. Em contrapartida, Mitchell et al nos trabalhos Mitchell e Vynnycky (2009) e Mitchell e Vynnycky (2012) apresentam aproximações para o problema via métodos de diferenças finitas, baseados nos métodos aqui apresentados: VSG e BIM.

\section{Extensões e outras aplicações}

\section{Aplicações}

Ablação é o processo em que massa é removida de um objeto através de vaporização ou similares processos erosivos. O exemplo clássico envolve a queima de meteoritos ou a queima da superfície de espaçonaves. Em veículos espaciais, a superfície exposta do material ablativo é desenhada para ser queimada no lançamento, e os gases resultantes adquirem muito da energia resultante da queima, enquanto o material serve de isolador. O processo pode ser considerado portanto um problema de Stefan de uma fase. Ablação também ocorre em outros ramos da fisica, como no derretimento ou sublimação de um sólido, ou perfurações a laser de metais ou da córnea.

\section{O problema de Stefan de duas fases}

O problema básico de duas fases é definido por um conjunto de equações que, por exemplo, descrevem o derretimento de um material sub-resfriado, onde o derretimento é dirigido pela temperatura especificada em $x=0$. Como $u(0, t)>u(s(t), t)>v(\infty, t)$, a frente de derretimento se move com velocidade positiva $s_{t}>0$. Assume-se que as propriedades termais permanecem constantes através da mudança de fase.

$$
\begin{gathered}
\frac{\partial u}{\partial t}=\frac{\partial^{2} u}{\partial x^{2}}, 0<x<s(t), \frac{\partial v}{\partial t}=\frac{\partial^{2} u}{\partial x^{2}}, x>s(t) \\
u=v=0, \beta \frac{d s}{d t}=\frac{\partial v}{\partial x}-\left.\frac{\partial u}{\partial x}\right|_{x=s} \\
u(0, t)=1, v(x, 0)=-1, \lim _{x \rightarrow \infty} v=-1
\end{gathered}
$$

Este sistema ainda possui uma solução exata

$$
\begin{gathered}
u=1-\frac{\operatorname{erf}\left(\frac{x}{2 \sqrt{t}}\right)}{\operatorname{erf}(\alpha)}, 0<x<s(t) \\
v=-1+\frac{\operatorname{erfc}\left(\frac{x}{2 \sqrt{t}}\right)}{\operatorname{erfc}(\alpha)}, s(t)<x<\infty
\end{gathered}
$$

em que a fronteira móvel satisfaz $s(t)=2 \alpha \sqrt{t}$ e $\alpha$ é determinado usando a condição de Stefan

$$
\beta \alpha \sqrt{\pi}=\frac{e^{-\alpha^{2}}}{\operatorname{erf} \alpha}-\frac{e^{-\alpha^{2}}}{\operatorname{erfc} \alpha}
$$

O método HBIM pode ser empregado naturalmente a cada fase, fazendo as integrações e utilizando-se de um perfil de aproximação adequado. É importante notar que novas condições de contorno devem ser aplicadas, pois há uma nova interface (e portanto uma nova correspondência) entre duas fases. 


\section{Incorporação de termos de fonte}

A incorporação de termos de fonte na equação do calor leva a uma extensão direta dos métodos integrais. O único ponto a ser notado é que, quando se desenvolve a condição de Stefan, agora deve-se levar em conta esse novo termo. Por exemplo, se

$$
u_{t}=u_{x x}+q(t)
$$

e $u(\delta(t), t)=1$, e $u_{x}(\delta(t), t)=0$, então em vez de usar $u_{x x}(\delta, t)=0$, encontra-se a nova condição $u_{x x}(\delta, t)+q(t)=0$.

\section{Extensões a dimensões maiores}

Outros domínios espaciais são estudados no trabalho de Caldwell e Chan (1998), incluindo esferas, cilindros, regiões anulares e regiões quadradas bidimensionais. Um empecilho comum a problemas nessas diversas regiões é a complexidade dos esquemas resultantes, que são difíceis de serem aplicados a problemas multidimensionais. O mesmo trabalho ainda introduz o método com formulação de entalpia, porém com a desvantagem de não se poder determinar precisamente a curva de mudança de fase. 
RESULTADOS E CONSIDERAÇÕES FINAIS 


\section{Referências Bibliográficas}

Bell e Abbas (1985) G.E. Bell e S.K. Abbas. Convergence properties of the heat balance integral method. Numerical Heat Transfer, 8(3):373-382. 2, 3, 15, 18, 32, 56, 57

Caldwell e Kwan (2004) J. Caldwell e YY Kwan. Numerical methods for one-dimensional stefan problems. Communications in numerical methods in engineering, 20(7):535-545.

Caldwell e Chan (1998) James Caldwell e Ching-Chuen Chan. Numerical solutions of the stefan problem by the enthalpy method and the heat balance integral method. Numerical Heat Transfer, 33(1):99-117. 67

Crank (1987) J. Crank. Free and moving boundary problems. Oxford University Press, USA. 59

Crepeau (2007) J. Crepeau. Josef stefan: His life and legacy in the thermal sciences. Experimental thermal and fluid science, 31(7):795-803. 3, 41

Evans (1998) Lawrence C Evans. Partial differential equations. 6

Furzeland (1980) RM Furzeland. A comparative study of numerical methods for moving boundary problems. IMA Journal of Applied Mathematics, 26(4):411-429. 59

Goodman (1958) T.R. Goodman. The heat-balance integral and its application to problems involving a change of phase. Trans. ASME, 80(2):335-342. iii, v, 1, 2, 7, 13, 45, 46, 47, 52, 58, 64

Hill (1987) J.M. Hill. One-dimensional Stefan problems: an introduction. Longman Scientific \& Technical.

Javierre et al. (2006) E. Javierre, C. Vuik, FJ Vermolen e S. Van der Zwaag. A comparison of numerical models for one-dimensional stefan problems. Journal of Computational and Applied Mathematics, 192(2):445-459.

Kutluay et al. (1997) S. Kutluay, AR Bahadir e A. Özdeş. The numerical solution of one-phase classical stefan problem. Journal of computational and applied mathematics, 81(1):135-144. 4, 60

Langford (1973) D. Langford. The heat balance integral method. International Journal of Heat and Mass Transfer, 16(12):2424-2428. 2, 3, 9, 13, 14, 54, 63

Mitchell (2011) SL Mitchell. An accurate nodal heat balance integral method with spatial subdivision. Numerical Heat Transfer, Part B: Fundamentals, 60(1):34-56. 2, 3, 4, 32, 33, 56, 64, 65

Mitchell e Myers (2010a) S.L. Mitchell e TG Myers. Application of standard and refined heat balance integral methods to one-dimensional stefan problems. SIAM review, 52(1):57-86. 2, 3, $47,52,64$ 
Mitchell e Myers (2010b) S.L. Mitchell e TG Myers. Improving the accuracy of heat balance integral methods applied to thermal problems with time dependent boundary conditions. International Journal of Heat and Mass Transfer, 53(17):3540-3551. 21

Mitchell e Myers (2012) SL Mitchell e TG Myers. Application of heat balance integral methods to one-dimensional phase change problems. International Journal of Differential Equations, 2012. $2,3,11,30,31,63$

Mitchell e Vynnycky (2009) SL Mitchell e M. Vynnycky. Finite-difference methods with increased accuracy and correct initialization for one-dimensional stefan problems. Applied Mathematics and Computation, 215(4):1609-1621. 64, 66

Mitchell e Vynnycky (2012) SL Mitchell e M. Vynnycky. An accurate finite-difference method for ablation-type stefan problems. Journal of Computational and Applied Mathematics. 66

Mosally et al. (2002) F. Mosally, AS Wood e A. Al-Fhaid. An exponential heat balance integral method. Applied mathematics and computation, 130(1):87-100. 2, 48

Mosally et al. (2005) F. Mosally, A.S. Wood e A. Al-Fhaid. On the convergence of the heat balance integral method. Applied mathematical modelling, 29(10):903-912. 3, 56, 65

Myers (2009) TG Myers. Optimizing the exponent in the heat balance and refined integral methods. International Communications in Heat and Mass Transfer, 36(2):143-147. 14, 25

Myers (2010) TG Myers. Optimal exponent heat balance and refined integral methods applied to stefan problems. International Journal of Heat and Mass Transfer, 53(5):1119-1127. 31

Myers e Mitchell (2011) TG Myers e S.L. Mitchell. Application of the combined integral method to stefan problems. Applied Mathematical Modelling, 35(9):4281-4294.

Myers et al. (2007) TG Myers, SL Mitchell, G. Muchatibaya e MY Myers. A cubic heat balance integral method for one-dimensional melting of a finite thickness layer. International Journal of Heat and Mass Transfer, 50(25):5305-5317. 47

Rubinshteĭn (1971) L.I. Rubinshter̆n. The Stefan Problem. Translations of Mathematical Monographs. American Mathematical Society. ISBN 9780821815779.

Sadoun et al. (2012) N. Sadoun, E.K. Si-Ahmed, P. Colinet e J. Legrand. On the boundary immobilization and variable space grid methods for transient heat conduction problems with phase change: Discussion and refinement. Comptes Rendus Mécanique.

Sadoun et al. (2006) Nacer Sadoun, El-Khider Si-Ahmed e Pierre Colinet. On the refined integral method for the one-phase stefan problem with time-dependent boundary conditions. Applied mathematical modelling, 30(6):531-544. 12

Savović e Caldwell (2009) S. Savović e J. Caldwell. Numerical solution of stefan problem with time-dependent boundary conditions by variable space grid method. Thermal Science, 13(4): 165-174. 4

Wood (2001) A.S. Wood. A new look at the heat balance integral method. Applied Mathematical Modelling, 25(10):815-824. 46, 47 


\section{Îndice Remissivo}

Stefan, 70 